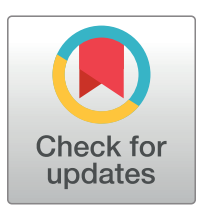

\section{f open access}

Citation: Galante J, Friedrich C, Dawson AF, Modrego-Alarcón M, Gebbing P, Delgado-Suárez I, et al. (2021) Mindfulness-based programmes for mental health promotion in adults in nonclinical settings: A systematic review and meta-analysis of randomised controlled trials. PLoS Med 18(1): e1003481. https://doi.org/10.1371/journal. pmed.1003481

Academic Editor: Vikram Patel, Harvard Medical School, UNITED STATES

Received: June 26, 2020

Accepted: December 10, 2020

Published: January 11, 2021

Peer Review History: PLOS recognizes the benefits of transparency in the peer review process; therefore, we enable the publication of all of the content of peer review and author responses alongside final, published articles. The editorial history of this article is available here: https://doi.org/10.1371/journal.pmed.1003481

Copyright: @ 2021 Galante et al. This is an open access article distributed under the terms of the Creative Commons Attribution License, which permits unrestricted use, distribution, and reproduction in any medium, provided the original author and source are credited.

Data Availability Statement: All data files are available from the University of Cambridge

RESEARCH ARTICLE

\title{
Mindfulness-based programmes for mental health promotion in adults in nonclinical settings: A systematic review and meta- analysis of randomised controlled trials
}

\begin{abstract}
Julieta Galante ${ }^{1,2 *}$, Claire Friedrich ${ }^{1}$, Anna F Dawson ${ }^{3}$, Marta Modrego-Alarcón ${ }^{4,5}$, Pia Gebbing ${ }^{6}$, Irene Delgado-Suárez ${ }^{4,7}$, Radhika Gupta ${ }^{1}{ }^{1}$, Lydia Dean ${ }^{1}$, Tim Dalgleish ${ }^{1,8}$, lan R White $\mathbb{i}^{9}$, Peter B Jones $\mathbb{D}^{1,2,8}$

1 University of Cambridge, Cambridge, United Kingdom, 2 National Institute for Health Research Applied Research Collaboration East of England, Cambridge, United Kingdom, 3 Australian National University, Canberra, Australia, 4 University of Zaragoza, Zaragoza, Spain, 5 Primary Care Prevention and Health Promotion Research Network (RedIAPP), Zaragoza, Spain, 6 Leiden University, Leiden, the Netherlands, 7 Institute of Medical Research Aragón, Zaragoza, Spain, 8 Cambridgeshire and Peterborough NHS Foundation Trust, Cambridge, United Kingdom, 9 University College London, London, United Kingdom
\end{abstract}

*mjg231@cam.ac.uk

\section{Abstract}

\section{Background}

There is an urgent need for mental health promotion in nonclinical settings. Mindfulnessbased programmes (MBPs) are being widely implemented to reduce stress, but a comprehensive evidence synthesis is lacking. We reviewed trials to assess whether MBPs promote mental health relative to no intervention or comparator interventions.

\section{Methods and findings}

Following a detailed preregistered protocol (PROSPERO CRD42018105213) developed with public and professional stakeholders, 13 databases were searched to August 2020 for randomised controlled trials (RCTs) examining in-person, expert-defined MBPs in nonclinical settings. Two researchers independently selected, extracted, and appraised trials using the Cochrane Risk-of-Bias Tool 2.0. Primary outcomes were psychometrically validated anxiety, depression, psychological distress, and mental well-being questionnaires at 1 to 6 months after programme completion. Multiple testing was performed using $p<0.0125$ (Bonferroni) for statistical significance. Secondary outcomes, meta-regression and sensitivity analyses were prespecified. Pairwise random-effects multivariate meta-analyses and prediction intervals (PIs) were calculated.

A total of 11,605 participants in 136 trials were included ( 29 countries, $77 \%$ women, age range 18 to 73 years). Compared with no intervention, in most but not all scenarios MBPs improved average anxiety (8 trials; standardised mean difference (SMD) $=-0.56 ; 95 \%$ confidence interval $(\mathrm{Cl})-0.80$ to $-0.33 ; p$-value $<0.001 ; 95 \% \mathrm{PI}-1.19$ to 0.06 ), depression (14 trials; $\mathrm{SMD}=-0.53 ; 95 \% \mathrm{Cl}-0.72$ to $-0.34 ; p$-value $<0.001 ; 95 \% \mathrm{PI}-1.14$ to 0.07 ), distress 
Repository (digital object identifier https://doi.org/ 10.17863/CAM.59644).

Funding: This publication presents independent research funded by the National Institute for Health Research (NIHR). The views expressed are those of the authors and not necessarily those of the NHS, the NIHR or the Department of Health and Social Care. JG is funded by a NIHR Post-doctoral Fellowship for this research project (salary and all project costs, PDF-2017-10-018, https://www.nihr. ac.uk), she is also supported by the NIHR Applied Research Collaboration (ARC) East of England (grant awarded to PBJ, RNAG/564, https://arc-eoe. nihr.ac.uk/). CF's salary for this research project was funded by a Cambridgeshire \& Peterborough NHS Foundation Trust grant awarded to JG (RNAG/552, https://www.cpft.nhs.uk/). IW was supported by the UK Medical Research Council (MC_UU_12023/21, https://mrc.ukri.org/). TD was supported by the UK Medical Research Council (SUAG/043 G101400, https://mrc.ukri.org/), the Wellcome Trust (104908/Z/14/Z, 107496/Z/15/Z, https://wellcome.org/), and the NIHR Cambridge Biomedical Research Centre (RG85446, 247730,

https://cambridgebrc.nihr.ac.uk/). PBJ is supported by the Wellcome Trust (095844/Z/11/Z, https:// wellcome.org/), the UK Medical Research Council (MR/N019067/1, https://mrc.ukri.org/), and the NIHR ARC East of England (RNAG/564, https://arceoe.nihr.ac.uk/). MMA and IDS were supported by the Spanish Ministry of Education, Culture and Sport (FPU15/00598 and FPU16/03565 respectively, http://www.mecd.gob.es/). The funders had no role in study design, data collection and analysis, decision to publish, or preparation of the manuscript.

Competing interests: The authors have declared that no competing interests exist.

\footnotetext{
Abbreviations: CES-D, Center for Epidemiologic Studies Depression Scale; $\mathrm{Cl}$, confidence interval; COVID-19, Coronavirus Disease 2019; GRADE, Grading of Recommendations Assessment, Development and Evaluation; ICC, intracluster correlation coefficient; ICTRP, International Clinical Trials Registry Platform; IPD, individual participant data; MBP, mindfulness-based programme; MBSR, Mindfulness-Based Stress Reduction; PANAS-P, Positive Affect Schedule; PI, prediction interval; PRISMA, Preferred Reporting Items for Systematic Reviews and Meta-Analyses; RCT, randomised controlled trial; SMD, standardised mean difference.
}

(27 trials; $\mathrm{SMD}=-0.45 ; 95 \% \mathrm{Cl}-0.58$ to $-0.31 ; p$-value $<0.001 ; 95 \% \mathrm{PI}-1.04$ to 0.14 ), and well-being ( 9 trials; SMD $=0.33 ; 95 \% \mathrm{Cl} 0.11$ to $0.54 ; p$-value $=0.003 ; 95 \% \mathrm{PI}-0.29$ to 0.94). Compared with nonspecific active control conditions, in most but not all scenarios MBPs improved average depression ( 6 trials; $S M D=-0.46 ; 95 \% \mathrm{Cl}-0.81$ to $-0.10 ; p$-value $=0.012,95 \% \mathrm{PI}-1.57$ to 0.66 ), with no statistically significant evidence for improving anxiety or distress and no reliable data on well-being. Compared with specific active control conditions, there is no statistically significant evidence of MBPs' superiority. Only effects on distress remained when higher-risk trials were excluded. USA-based trials reported smaller effects. MBPs targeted at higher-risk populations had larger effects than universal MBPs. The main limitation of this review is that confidence according to the Grading of Recommendations Assessment, Development and Evaluation (GRADE) approach is moderate to very low, mainly due to inconsistency and high risk of bias in many trials.

\section{Conclusions}

Compared with taking no action, MBPs of the included studies promote mental health in nonclinical settings, but given the heterogeneity between studies, the findings do not support generalisation of MBP effects across every setting. MBPs may have specific effects on some common mental health symptoms. Other preventative interventions may be equally effective. Implementation of MBPs in nonclinical settings should be partnered with thorough research to confirm findings and learn which settings are most likely to benefit.

\section{Author summary}

\section{Why was this study done?}

- Mindfulness courses to increase well-being and reduce stress have become very popular; most are in community settings.

- Many randomised controlled trials (RCTs) tested whether mindfulness courses show benefit, but results are varied and, to our knowledge, there are no reviews combining the data from these studies to show an overall effect.

\section{What did the researchers do and find?}

- Worldwide, we identified 136 RCTs on mindfulness training for mental health promotion in community settings. We reviewed them all, assessed their quality, and calculated their combined effects.

- We showed that, compared with doing nothing, mindfulness reduces anxiety, depression, and stress, and increases well-being, but we cannot be sure that this will happen in every community setting.

- In these RCTs, mindfulness is neither better nor worse than other feel-good practices such as physical exercise, and RCTs in this field tend to be of poor quality, so we cannot be sure that our combined results represent the true effects. 


\section{What do these findings mean?}

- Mindfulness courses in the community need to be implemented with care, because we cannot assume that they work for everyone, everywhere.

- We need good quality collaborative research to find out which types of communities benefit from the different types of mindfulness courses available.

- The courses that work best may be those aimed at people who are most stressed or in stressful situations.

\section{Introduction}

With major depression listed as the second largest cause of global years lived with disability, and several other mental disorders within the top 25 [1], there is a widely recognised need to prioritise preventative programmes [2]. Such programmes, introduced across schools, workplaces, and communities, usually target psychological distress which, if unaddressed, can result in mental and physical disorders [3]. The public is willing to take preventative action: 65\% would practice something for 15 minutes per day if that could reduce their stress by $30 \%$ [4].

Commonly implemented preventative interventions include mindfulness-based programmes (MBPs), which typically define mindfulness as "the awareness that emerges through paying attention on purpose, in the present moment, and nonjudgmentally to the unfolding of experience moment by moment" [5]. Recently, a group of prominent mindfulness teachers have agreed on what MBPs should comprise: sustained training in formal and informal mindfulness meditation, scientific approaches to managing health, suitability for delivery in public institutions across a range of settings and cultures, and class-based experiences of collective and individual inquiry with a qualified teacher in a participatory learning process [6]. The United Kingdom National Health Service offers therapies that are based on mindfulness [7]. However, the cultural traditions from which mindfulness stems do not conceptualise it as a therapy [8]; this has encouraged advocates, first in the United States of America (USA) and thereafter more globally, to widely promote nontherapeutic secular mindfulness training [9]. Currently, in the USA, twice as many people practice mindfulness for wellness than for treating a specific health condition [10]. MBPs, ubiquitous in high-income countries, are frequently promoted as the go-to universal tool to reduce stress and increase well-being, accessible to anyone, anywhere [8].

Trials assessing MBPs in nonclinical settings have quickly accumulated in recent years. Systematic reviews have synthesised findings from MBPs from educators [11,12], parents [13], caregivers [14,15], healthcare professionals [16-21], athletes [22], working adults [23-27], older adults [28], university students [29-31], and the general population [32-36], primarily focusing on wellness and mental health outcomes. Most, but by no means all results favour MBPs over comparison conditions. These reviews tend towards broad inclusion criteria, reflecting the state of the science at the time they were conducted. As well as gold standard randomised controlled trials (RCTs), both uncontrolled and nonrandomised trial findings are many times included. In contrast, literature searches often then exclude important areas of grey literature such as unpublished RCTs, as well as studies in languages other than English. Some reviews also combine both nonclinical and clinical MBPs or include other interventions, 
and methods are not prespecified. Finally, formal meta-analysis of the synthesised data is infrequent and sometimes (but not always, e.g., $[26,37]$ ) neglects to disaggregate trials with active versus passive control groups.

There is now a critical mass of good quality RCTs of MBPs in nonclinical settings. Consequently, in line with calls to improve mindfulness research quality, we conducted a comprehensive systematic review and meta-analysis of MBPs in nonclinical settings targeted at mental health that focused only on RCT data $[38,39]$. Our primary question was whether these MBPs improve anxiety, depression, psychological distress, and/or well-being 1 to 6 months after programme completion, relative to no intervention or comparator interventions.

\section{Methods}

Our review procedures were developed with public and professional stakeholders [40,41] and published in detail in a prospective protocol [42]. Stakeholders shaped the research questions, prioritised outcomes and moderation analyses, selected studies, extracted data, interpreted results, and developed lay summaries. This study is reported as per the Preferred Reporting Items for Systematic Reviews and Meta-Analyses (PRISMA) guideline (S1 Checklist) [43].

\section{Study search and selection}

Thirteen databases were electronically searched for eligible studies from inception to 1 August 2020: AMED, ASSIA, CENTRAL, CINAHL, ERIC, EThOS, EMBASE, MEDLINE, ProQuest, PsycINFO, Scopus, Web of Science, and the World Health Organization International Clinical Trials Registry Platform (ICTRP). No geographical, language, or publication date/type restrictions were applied (S1 Appendix). Publication references were inspected for further studies. Unpublished reports were included (e.g., theses). Nonpublic sources of studies (e.g., private datasets) were not sought [44], but authors were contacted to seek clarification or data from which effect sizes could be calculated if such data were not available in their report. We searched ICTRP to find further trials and to assess publication bias.

Studies were deemed eligible if they: (1) were parallel-arm RCTs including cluster-RCTs; (2) assessed group-based first-generation MBPs as defined in Crane [6], with a minimum intensity of 4 one-hour in-person teacher-led sessions or equivalent (4 MBP sessions were used as the "minimum dose" for participants in previous studies [45], and 1-hour sessions are common in nonclinical busy settings [46]); (3) included adult (18+ years old) participants living in the community, as long as the trial had not selected them for having any particular clinical condition (MBPs targeting specific community groups were included); (4) reported at least one of the prespecified outcomes of interest (see below); and (5) compared MBPs with a control group (i.e., not just with a different type of eligible MBP). Online MBPs were excluded as we believe they are different enough from in-person MBPs (e.g., typically not group-based, and fully or semiautomated) to merit their own separate analysis [47].

Using Covidence software [48], 2 reviewers independently assessed the titles and abstracts of retrieved records against inclusion criteria. Full texts were obtained for abstracts not deemed irrelevant by both and again independently assessed for eligibility. Multiple reports of the same trial were combined. Two researchers independently extracted the information from the included full-text papers using prepiloted forms (S1 Appendix). Disagreements were discussed and resolved within the review team.

\section{Outcomes: Organisation, assessment, and transformation}

The 4 primary outcome domains were anxiety, depression, psychological distress, and mental well-being, measured in a primary time range of between 1 and 6 months following 
programme completion. Measures taken less than 1 month after programme completion may not inform stable changes making them less clinically relevant, so this "post-intervention" time range was considered as a secondary outcome, as was the time range of follow-ups longer than 6 months post-intervention. Other secondary outcome domains included cognitive functioning (assessed using experimental tasks), real life functioning (e.g., professional performance), relationship with the self (e.g., self-esteem, self-compassion), and psychosomatic outcomes (e.g., sleep, pain). Adverse event or effect data were recorded. In view of the high number of trials reporting dispositional mindfulness, we included it as a mechanistic outcome, although it is not in the review protocol. Outcomes deemed not to belong to any of the outcome domains described above were excluded from the review. All self-reported outcomes had to be psychometrically validated in the language used and could not just measure momentary states [49]. If a study measured an outcome more than once within these prespecified time ranges, the longer follow-up was used. When trials reported more than 1 measure of the same outcome within the same time range, or more than 1 eligible sample, we applied the prioritisation criteria set out prospectively in our protocol [42]. For example, we preferred trial primary outcomes and intention-to-treat samples. Trial outcomes were preliminarily categorised into the review outcome domains before analysis via discussion between reviewers extracting the data, with final categorisation made by senior team members blind to trial results and to which trial measures belonged (S1 Appendix).

The standardised mean difference adjusted for small sample bias (SMD, or Hedges' g) was used as a measure of treatment effect [50]. When baseline outcome values were reported, we calculated SMD using the ANCOVA estimate [51]. When missing from trial reports, withinstudy baseline-endpoint correlations were calculated from publicly available individual participant data (IPD) or imputed as follows. For distress and well-being outcomes, we assumed that within-study baseline-endpoint correlations for each time point were the same as in the IPD from a trial recently conducted by some of us and included in this review [52]. For the other outcomes, we took the mean of the correlations available in other studies. When baseline data were not available, we calculated SMD using adjusted (if available) or unadjusted final values analyses [53-55]. Missing standard deviations were imputed averaging those of other time points within the same study and outcome, or, if not available, from other studies using the same instrument. Subscales were combined when possible using their correlations. Ordinal and categorical data were transformed to be pooled together with continuous data [42]. When outcome sample size was missing, it was estimated from other data. We accounted for clustering when this was missing in cluster-RCT reports [42].

Control groups were grouped into categories following related reviews to facilitate comparison [56,57]: (1) no intervention or wait-list ("passive controls"); (2) interventions designed principally to take account of nonspecific therapeutic factors such as receiving attention from a teacher, without expected specific effects on outcomes of interest ("active nonspecific controls"); and (3) interventions with active ingredients specifically designed to augment change in our outcomes ("active specific controls"). When trials had multiple control groups fitting 1 category, these were combined. In multiarm trials with $2 \mathrm{MBPs}$, these were combined.

Two researchers independently assessed trials' methodological quality for the included outcomes using the Cochrane revised tool for assessing risk of bias in randomised trials (RoB2, version 9 October 2018) for RCTs and cluster-RCTs [58]. This tool stringently measures potential bias across 5 sources (called "domains" in the tool): (1) randomisation; (2) deviations from intended interventions; (3) missing outcome data; (4) measurement of the outcome; and (5) selection of the reported result. None of the authors assessed risk of bias of their own trial. When data were unavailable for outcomes mentioned in trials' public registers or publications, this was interpreted as known nonreporting bias. Potentially eligible trial registry records with 
no published results were considered suggestive of nonreporting bias. We attempted to contact authors if trial enrolment started more than 3 years before our search date and deemed a trial as unpublished if authors offered either no outcome reports or an account of their absence [59]. Small-study effects suggesting unknown nonreporting and other biases were assessed by visual inspection of funnel plots in meta-analyses of primary outcome domains with at least 10 studies. We used the Grading of Recommendations Assessment, Development and Evaluation (GRADE) approach to assess confidence in the cumulative evidence [60]. It classifies the quality of evidence for each result in 1 of 4 levels of certainty-high, moderate, low, and very low. For each primary outcome we considered trials' risk of bias, meta-analysis nonreporting bias, imprecision (confidence intervals (CIs)) inconsistency (prediction intervals (PIs)), and indirectness of evidence.

\section{Data synthesis}

We used Stata/SE 16.1 [61] to compute pairwise random-effects meta-analyses within comparator categories and applied a conservative Bonferroni correction for multiple testing to each of the 4 primary outcome domains using $p<0.0125$ as the critical level for significance to maintain the overall type I error rate at 0.05 [62]. We included the 4 primary outcome domains in a multivariate meta-analysis using all the prespecified time point ranges available (i.e., post-intervention, 1 to 6 months later, over 6 months later). Multivariate meta-analysis differs from univariate meta-analysis in that it takes into account within-and between-study correlations, reducing bias, and improving precision [63]. Stata's mvmeta package was employed $[64,65]$. Within-study correlations between outcome domains were estimated from our IPD and assumed to apply to the other studies [52]. Between-study variance-covariance matrices were estimated as unstructured using restricted maximum likelihood; if not possible, they were estimated as exchangeable with the fixed correlation that yielded the largest restricted log likelihood.

Multivariate meta-analyses for secondary outcome domains also included all available time point ranges, but data from our IPD were less suitable to estimate between-outcome withinstudy correlations and no IPD were available, so meta-analyses were outcome-specific. Within-study correlations were bypassed using Riley's method as our IPD were unsuitable [66]; for cognitive functioning, this method had to be rejected due to extreme correlations, so a within-study correlation of 0.75 was imputed (high given the outcome-specific analysis) with a sensitivity analysis testing 0.5 [67]. Results of meta-analyses containing few studies were interpreted cautiously, including multivariate meta-analyses with outcomes derived from a single trial. When multivariate meta-analyses failed to converge, results of univariate meta-analyses were reported. As a measure of real-life implications of between-study heterogeneity, prediction intervals were estimated reflecting the variation in intervention effects over the different trial settings [68,69].

We conducted prespecified sensitivity analyses on primary outcome domains where we had data from at least 10 studies. These explored sensitivity of results to (1) overall and biassource-specific risks of bias, by removing trials with higher risk of bias; (2) within-study correlation assumptions, by using Riley's estimation method, and by conducting univariate metaanalyses; (3) standard deviation imputations, by inflating them by 10\%; (4) imputing intracluster correlation coefficients (ICCs), by using ICC $=0.10$; and (5) skewed data, by excluding data coming from samples of fewer than 30 . Post hoc sensitivity analyses were also conducted as outlined in the Results.

We conducted prespecified moderator analyses on primary outcome domains of the following study-level characteristics: (1) region, comparing trials from the USA-where MBPs are 
most established in nonclinical settings-with the rest of the world; (2) type of participant, grouping interventions into universal (for anyone), selective (for those at higher risk of developing mental health problems, such as carers), or indicated (for individuals with subclinical symptoms of mental health conditions) [70]; (3) intervention duration; (4) additional inclusion of intervention components other than the activities common to all MBPs [6]; and (5) active control type. We conducted these only when there were at least 10 studies with moderator information. We used random-effects multivariable meta-regression within multivariate meta-analyses and interpreted with caution analyses of categorical subgroup variables with fewer than 5 studies per category [71].

\section{Results}

A study selection flowchart is shown in Fig 1 . Too much information was missing to assess eligibility when only conference abstracts were available from databases or authors (S1 Appendix). MBP teachers are required to be well trained, but many trial reports do not describe their credentials [6]. We (1) included these studies, excluding only those explicitly mentioning insufficient training; and (2) conducted an ad hoc sensitivity analysis only including studies which suggested criteria-concordant training ( 97 trials, $71 \%$ ) to see if results would differ.

\section{Study characteristics}

One hundred and thirty-six trials were eligible for meta-analysis, 129 participant-level RCTs, and 7 cluster-RCTs. Table 1 summarises the main characteristics of the included studies. Trials were conducted between 1997 and 2020 across 29 countries. Almost half of the trials were completed in North America (mainly USA), 37 in Europe, 19 in Asia (mainly China), 6 in the Middle East, 5 in Australia, and 4 in South America. Sample sizes varied from 18 to 616 participants with a median of 60 . Mean ages ranged from 18 to 73 years old, and the gender balance differed between trials with a mean of $77 \%$ women. Sixteen trials (12\%) recruited stressed individuals for whom the MBP was considered an indicated preventative intervention. MBPs were selective interventions in 47 trials (35\%), targeting groups such as healthcare workers, medical interns, carers, school teachers, and pregnant women. The remaining 73 trials (54\%) used "universal" self-selected samples like community adults, students, employees, or older adults. Those with severe mental health problems or recent stressful life events were excluded in 99 trials $(73 \%)$.

MBPs were optional courses in all of the settings. The most common MBP was Mindfulness-Based Stress Reduction (MBSR) [5], sometimes slightly adapted, assessed in 44 (32\%) trials. The most common additional component was physical activity (60 studies, $44 \%$ ). MBP group sizes ranged from 6 to 30 participants per group. Planned intervention contact hours ranged from 4 to 30 hours with a mean of 16 . Information about MBP teachers typically lacked detail (e.g., teacher background).

Trials measured a wide range of outcomes within our domains of interest. The most common primary outcome measures were: for anxiety the Beck Anxiety Inventory, for depression the Center for Epidemiologic Studies Depression Scale (CES-D), for psychological distress the Perceived Stress Scale, and for mental well-being the Positive Affect Schedule (PANAS-P). Psychological distress was the most commonly measured outcome domain (102 trials, 75\%). All of the outcome measures were self-reported except for some real-life functioning outcomes such as exams, some psychosomatic outcomes such as peri-labour opioid use, and all cognitive functioning outcomes which involved experimental tasks. Follow-up times ranged from post-intervention (most trials) to an outlier of 6 years [72]. The most common control group was passive (no intervention or waitlist), used in 96 trials (71\%). 


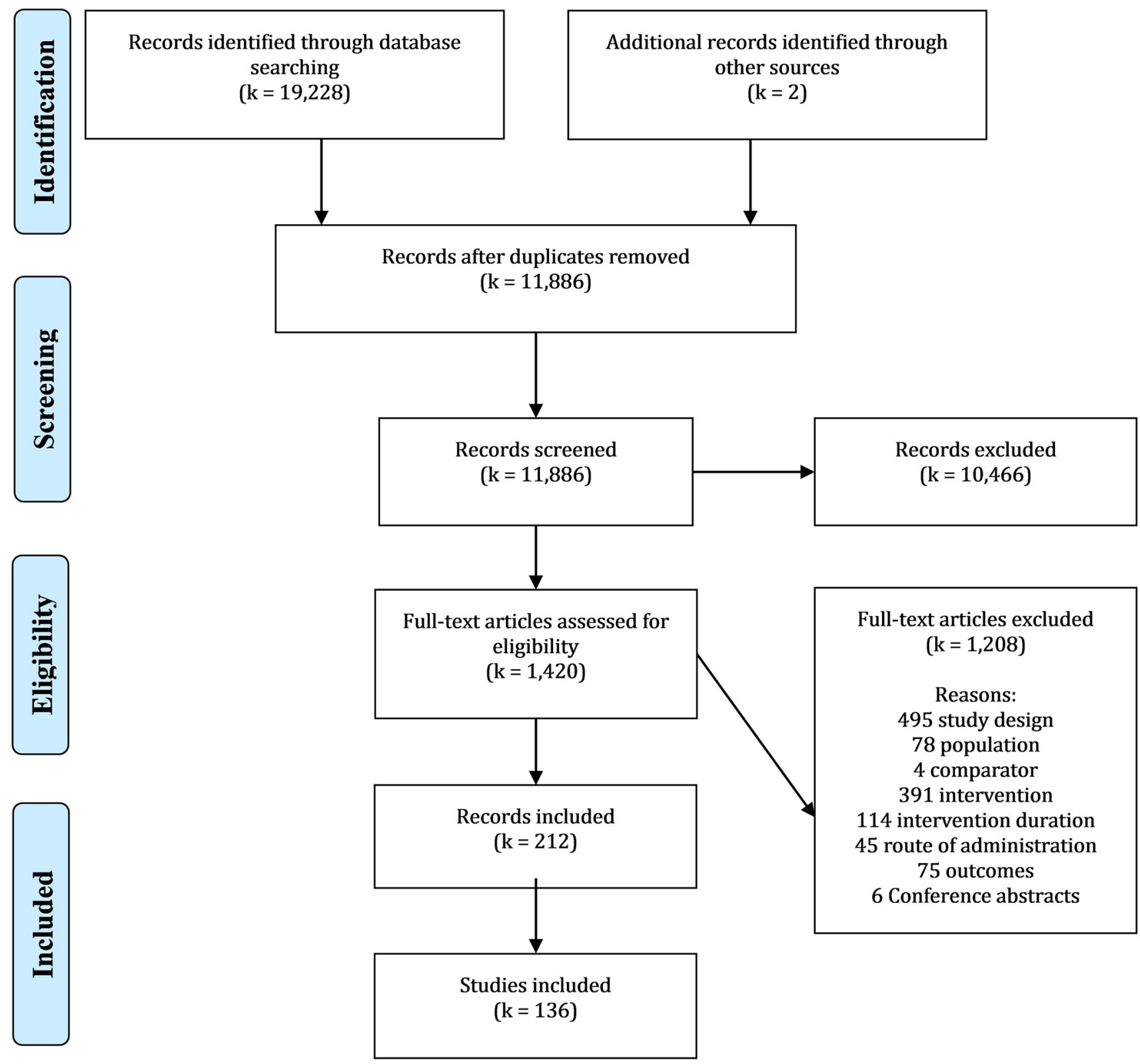

Fig 1. Study selection flowchart. * Reasons for full-text exclusion are listed in the order they were assessed. https://doi.org/10.1371/journal.pmed.1003481.g001

\section{Outcomes}

As an initial check, we undertook univariate meta-analyses (S1 Appendix). Below, we report primary outcome results of the multivariate meta-analyses; secondary outcomes (i.e., other time point ranges, and cognitive functioning, real life functioning, relationship with the self, and psychosomatic outcomes measured at all time point ranges) are reported in S1 Appendix. When reading this report, outcome improvement or deterioration should be understood as relative to the control group, not to baseline.

In comparison with passive control groups and between 1 and 6 months post-intervention, on average MBPs improved anxiety $(\mathrm{SMD}=-0.56 ; 95 \% \mathrm{CI}-0.80$ to -0.33 ; $p$-value $<0.001$; 
Table 1. Characteristics of included studies.

\begin{tabular}{|c|c|c|c|c|c|c|c|c|}
\hline $\begin{array}{l}\text { First Author, } \\
\text { Year, Country }\end{array}$ & $N$ & $\begin{array}{l}\text { Participants } \\
\text { (category) }\end{array}$ & $\begin{array}{l}\text { Mean Age } \\
\text { (SD) }\end{array}$ & Women & Outcomes & Intervention (category) & Control/s (category) & $\begin{array}{l}\text { Outcome Time } \\
\text { Points }\end{array}$ \\
\hline $\begin{array}{l}\text { Aeamla-Or } 2015 \\
\text { [73], Thailand }\end{array}$ & 127 & Nursing students (1) & $19(0.9)$ & $91 \%$ & $\begin{array}{l}\text { Dep, Dis, } \\
\text { Mindf, Real } \\
\text { func, Self }\end{array}$ & MBSR (3) & $\begin{array}{l}\text { No intervention (support } \\
\text { as usual) (1) }\end{array}$ & $\begin{array}{l}\text { Post-int, } 2 \mathrm{~m} \text {, } \\
6 \mathrm{~m}\end{array}$ \\
\hline $\begin{array}{l}\text { Agee } 2009[74], \\
\text { US }\end{array}$ & 43 & Community adults (1) & $42(13)$ & $91 \%$ & Dis, Mindf & Mindfulness meditation (1) & $\begin{array}{l}\text { Progressive Muscle } \\
\text { Relaxation (3) }\end{array}$ & $\begin{array}{l}\text { Post-int, } 1 \mathrm{~m} \text {, } \\
3 \mathrm{~m}\end{array}$ \\
\hline $\begin{array}{l}\text { Allen } 2012 \text { [75], } \\
\text { Denmark }\end{array}$ & 61 & Community adults (1) & 27 & $55 \%$ & $\operatorname{Cog}$ & Mindfulness training (1) & $\begin{array}{l}\text { Shared Reading and } \\
\text { Learning }(2)\end{array}$ & Post-int \\
\hline $\begin{array}{l}\text { Amutio } 2015 \\
{[76,77], \text { Spain }} \\
\end{array}$ & 42 & Physicians (2) & $47(9.4)$ & $57 \%$ & $\begin{array}{l}\text { Mindf, Real } \\
\text { func }\end{array}$ & MBSR (3) & Waitlist (1) & Post-int \\
\hline $\begin{array}{l}\text { Anclair } 2018[78], \\
\text { Sweden }\end{array}$ & 21 & $\begin{array}{l}\text { Parents of chronically } \\
\text { ill children (2) }\end{array}$ & $41(6.1)$ & $93 \%$ & Dis, Real func & $\begin{array}{l}\text { Here and Now Version } 2.0 \\
(2)\end{array}$ & CBT intervention (3) & Post-int \\
\hline $\begin{array}{l}\text { Anderson } 2007 \\
\text { [79], Canada }\end{array}$ & 86 & Healthy adults (1) & 39 & $92 \%$ & Anx, Cog, Dep & MBSR (3) & Waitlist (1) & Post-int \\
\hline $\begin{array}{l}\text { Armstrong } 2016 \\
\text { [80], UK }\end{array}$ & 34 & $\begin{array}{l}\text { Stressed university } \\
\text { students and staff (3) }\end{array}$ & $30(8.4)$ & $91 \%$ & $\begin{array}{l}\text { Anx, Dep, } \\
\text { Mindf, Real } \\
\text { func, Self }\end{array}$ & MBCT (2) & $\begin{array}{l}\text { "Get Self-Help" online } \\
\text { course (2) }\end{array}$ & $1 \mathrm{~m}$ \\
\hline $\begin{array}{l}\text { Arredondo } 2017 \\
\text { [81], Spain }\end{array}$ & 40 & Employees (1) & $37(5.6)$ & $78 \%$ & $\begin{array}{l}\text { Dis, Mindf, } \\
\text { Real func, Self }\end{array}$ & $\begin{array}{l}\text { Brief integrated mindfulness } \\
\text { practices (2) }\end{array}$ & Waitlist (1) & Post-int, $3 \mathrm{~m}$ \\
\hline $\begin{array}{l}\text { Astin } 1997 \text { [82], } \\
\text { US }\end{array}$ & 28 & $\begin{array}{l}\text { Undergraduate health } \\
\text { students (1) }\end{array}$ & NA & $64 \%$ & Dis & MBSR (3) & Waitlist (1) & Post-int \\
\hline $\begin{array}{l}\text { Asuero } 2014[83], \\
\text { Spain }\end{array}$ & 68 & $\begin{array}{l}\text { Primary care health } \\
\text { professionals }(2)\end{array}$ & $47(8)$ & $92 \%$ & $\begin{array}{l}\text { Mindf, Real } \\
\text { func }\end{array}$ & MBSR (3) & Waitlist (1) & Post-int \\
\hline $\begin{array}{l}\text { Auseron } 2018 \\
\text { [84], Spain }\end{array}$ & 48 & $\begin{array}{l}\text { Primary care health } \\
\text { professionals (2) }\end{array}$ & $50(8.2)$ & $84 \%$ & $\begin{array}{l}\text { Dis, Mindf, } \\
\text { Real func, Self }\end{array}$ & $\begin{array}{l}\text { Mindfulness and self- } \\
\text { compassion (2) }\end{array}$ & No intervention (1) & Post-int \\
\hline $\begin{array}{l}\text { Barrett } 2012 \text { [85- } \\
\text { 91], US }\end{array}$ & 154 & Older adults (2) & $59(6.6)$ & $82 \%$ & $\begin{array}{l}\text { Dis, WB, } \\
\text { Mindf, Somat, } \\
\text { Real func }\end{array}$ & Mindfulness meditation (3) & $\begin{array}{l}\text { a) Waitlist (1) b) Exercise } \\
\text { program (3) }\end{array}$ & Post-int, $3 \mathrm{~m}$ \\
\hline $\begin{array}{l}\text { Barrett } 2018[92- \\
97], \text { US }\end{array}$ & 413 & $\begin{array}{l}\text { Adults aged } 30 \text { to } 69 \\
\text { years (1) }\end{array}$ & $50(11.6)$ & $76 \%$ & $\begin{array}{l}\text { Dep, Dis, WB, } \\
\text { Mindf, Somat, } \\
\text { Real func }\end{array}$ & $\operatorname{MBSR}(3)$ & $\begin{array}{l}\text { a) No intervention (1) b) } \\
\text { Progressive moderate } \\
\text { intensity exercise (3) }\end{array}$ & $\begin{array}{l}\text { Post-int, } 2 \mathrm{~m} \text {, } \\
3 \mathrm{~m}, 6 \mathrm{~m}\end{array}$ \\
\hline $\begin{array}{l}\text { Beattie } 2017 \text { [98], } \\
\text { Australia }\end{array}$ & 48 & Pregnant women (2) & 29 & $100 \%$ & $\begin{array}{l}\text { Dep, Dis, } \\
\text { Mindf }\end{array}$ & $\begin{array}{l}\text { Mindfulness in Pregnancy } \\
\text { (2) }\end{array}$ & $\begin{array}{l}\text { Pregnancy Support Active } \\
\text { Control Intervention } \\
\text { (PSP) (3) }\end{array}$ & Post-int, $1.5 \mathrm{~m}$ \\
\hline $\begin{array}{l}\text { Behbahani } 2018 \\
\text { [99], Iran }\end{array}$ & 60 & Parents/carers (2) & NA & $100 \%$ & Dis & $\begin{array}{l}\text { Mindful Parenting Training } \\
\text { (1) }\end{array}$ & No intervention (1) & Post-int, $2 \mathrm{~m}$ \\
\hline $\begin{array}{l}\text { Benn } 2012[100] \text {, } \\
\text { US }\end{array}$ & 70 & $\begin{array}{l}\text { Parents and educators } \\
\text { of children with } \\
\text { special needs ( } 2 \text { ) }\end{array}$ & $\begin{array}{l}\text { Range 26- } \\
60\end{array}$ & $92 \%$ & $\begin{array}{l}\text { Dep, Dis, WB, } \\
\text { Mindf, Self }\end{array}$ & $\begin{array}{l}\text { Stress Management and } \\
\text { Relaxation Techniques in } \\
\text { Education (2) }\end{array}$ & Waitlist (1) & Post-int, $2 \mathrm{~m}$ \\
\hline $\begin{array}{l}\text { Berghmans } 2010 \\
\text { [101], France }\end{array}$ & 26 & Students (1) & $28(5.8)$ & $78 \%$ & Anx, Dep & MBSR (3) & No intervention (1) & Post-int \\
\hline $\begin{array}{l}\text { Black } 2015 \text { [102- } \\
\text { 106], US }\end{array}$ & 49 & $\begin{array}{l}\text { Older adults with } \\
\text { sleep disturbance (3) }\end{array}$ & $66(7.4)$ & $67 \%$ & $\begin{array}{l}\text { Anx, Dep, Dis, } \\
\text { Mindf, Somat }\end{array}$ & MAPs (1) & $\begin{array}{l}\text { Sleep Hygiene and } \\
\text { Education (3) }\end{array}$ & Post-int \\
\hline $\begin{array}{l}\text { Brown } 2016 \text { [107], } \\
\text { US }\end{array}$ & 38 & Carers (2) & $61(10.4)$ & $84 \%$ & Dis, Real func & MBSR (3) & $\begin{array}{l}\text { Near-structurally } \\
\text { equivalent to standard } \\
\text { Social Support (2) }\end{array}$ & Post-int, $3 \mathrm{~m}$ \\
\hline $\begin{array}{l}\text { Carmody } 2011 \\
\text { [108], US }\end{array}$ & 110 & $\begin{array}{l}\text { Peri/postmenopausal } \\
\text { women (2) }\end{array}$ & $53(4.9)$ & $100 \%$ & $\begin{array}{l}\text { Anx, Dep, Dis, } \\
\text { Mindf, Somat }\end{array}$ & MBSR (3) & Waitlist (1) & Post-int, $3 \mathrm{~m}$ \\
\hline $\begin{array}{l}\text { Carson } 2004 \\
{[109,110], \text { US }}\end{array}$ & 114 & $\begin{array}{l}\text { Non-distressed } \\
\text { couples (1) }\end{array}$ & $38(11.4)$ & $50 \%$ & Dis & $\begin{array}{l}\text { Mindfulness-Based } \\
\text { Relationship Enhancement } \\
\text { (2) }\end{array}$ & Waitlist (1) & Post-int, $3 \mathrm{~m}$ \\
\hline $\begin{array}{l}\text { Cerna } 2019 \text { [111], } \\
\text { Chile }\end{array}$ & 103 & General adults (1) & 31 & $75 \%$ & Dep, WB & $\begin{array}{l}\text { Brief mindfulness training } \\
\text { program (1) }\end{array}$ & Waitlist (1) & Post-int \\
\hline
\end{tabular}


Table 1. (Continued)

\begin{tabular}{|c|c|c|c|c|c|c|c|c|}
\hline $\begin{array}{l}\text { First Author, } \\
\text { Year, Country }\end{array}$ & $N$ & $\begin{array}{l}\text { Participants } \\
\text { (category) }\end{array}$ & $\begin{array}{l}\text { Mean Age } \\
(\mathrm{SD})\end{array}$ & Women & Outcomes & Intervention (category) & Control/s (category) & $\begin{array}{l}\text { Outcome Time } \\
\text { Points }\end{array}$ \\
\hline $\begin{array}{l}\text { Christopher } 2018 \\
\text { [112-114], US }\end{array}$ & 61 & $\begin{array}{l}\text { Law enforcement } \\
\text { officers (2) }\end{array}$ & $44(6.1)$ & $13 \%$ & $\begin{array}{l}\text { Anx, Cog, Dep, } \\
\text { Dis, Somat, } \\
\text { Real func, Self }\end{array}$ & $\begin{array}{l}\text { Mindfulness-Based } \\
\text { Resilience Training (1) }\end{array}$ & Waitlist (1) & Post-int, $3 \mathrm{~m}$ \\
\hline $\begin{array}{l}\text { Cohen-Katz } 2004 \\
{[115,116], \text { US }}\end{array}$ & 27 & $\begin{array}{l}\text { Healthcare workers } \\
(2)\end{array}$ & 46 & $100 \%$ & $\begin{array}{l}\text { Dis, Mindf, } \\
\text { Real func }\end{array}$ & MBSR (3) & Waitlist (1) & Post-int \\
\hline $\begin{array}{l}\text { Corsica } 2014 \\
\text { [117], US }\end{array}$ & 53 & $\begin{array}{l}\text { People struggling with } \\
\text { emotional eating ( } 3 \text { ) }\end{array}$ & $45(10.4)$ & $98 \%$ & Dis, Real func & $\begin{array}{l}\text { a) MBSR merged with b) } \\
\text { MBSR plus Stress-eating } \\
\text { intervention (3) }\end{array}$ & $\begin{array}{l}\text { Stress-eating intervention } \\
\text { (3) }\end{array}$ & Post-int, $1.5 \mathrm{~m}$ \\
\hline $\begin{array}{l}\text { Cousin } 2016 \\
\text { [118], UK }\end{array}$ & 87 & Adults (1) & $49(13)$ & $77 \%$ & Dis, WB & MBCT (2) & Waitlist (1) & Post-int \\
\hline $\begin{array}{l}\text { Damião Neto } \\
2019 \text { [119], Brazil }\end{array}$ & 141 & Medical students (1) & 19 & $50 \%$ & $\begin{array}{l}\text { Anx, Dep, Dis, } \\
\text { Mindf }\end{array}$ & Mindfulness meditation (1) & $\begin{array}{l}\text { "Introduction to } \\
\text { University Life" classes (2) }\end{array}$ & Post-int \\
\hline $\begin{array}{l}\text { Davidson } 2003 \\
\text { [120], US }\end{array}$ & 48 & Healthy employees (1) & 36 & $71 \%$ & Anx, Dis, WB & MBSR (3) & Waitlist (1) & Post-int, $4 \mathrm{~m}$ \\
\hline $\begin{array}{l}\text { Delgado } 2010 \\
\text { [121], Spain }\end{array}$ & 36 & $\begin{array}{l}\text { Female university } \\
\text { students (1) }\end{array}$ & $\begin{array}{l}\text { Range 18- } \\
24\end{array}$ & $100 \%$ & $\begin{array}{l}\text { Anx, Dep, Dis, } \\
\text { WB }\end{array}$ & Mindfulness training (1) & $\begin{array}{l}\text { Relaxation training } \\
\text { programme (3) }\end{array}$ & Post-int \\
\hline $\begin{array}{l}\text { Delgado-Pastor } \\
2015 \text { [122], Spain }\end{array}$ & 45 & $\begin{array}{l}\text { Female university } \\
\text { students (1) }\end{array}$ & $22(3.9)$ & $100 \%$ & $\begin{array}{l}\text { Dep, Dis, WB, } \\
\text { Mindf }\end{array}$ & $\begin{array}{l}\text { a) Mindfulness cognitive } \\
\text { training merged with } b \text { ) } \\
\text { Mindfulness interoceptive } \\
\text { training group (1) }\end{array}$ & No intervention (1) & Post-int \\
\hline $\begin{array}{l}\text { Desbordes } 2012 \\
\text { [123], US }\end{array}$ & 51 & Healthy adults (1) & $34(7.7)$ & $62 \%$ & Anx, Dep & $\begin{array}{l}\text { Mindful attention training } \\
\text { (1) }\end{array}$ & $\begin{array}{l}\text { a) Cognitively-Based } \\
\text { Compassion Training ( } 3 \text { ) } \\
\text { merged with b) Health } \\
\text { discussion (3) }\end{array}$ & Post-int \\
\hline $\begin{array}{l}\text { De Vibe 2013 } \\
{[72,124-128] \text {, }} \\
\text { Norway }\end{array}$ & 293 & $\begin{array}{l}\text { Health undergraduate } \\
\text { students (1) }\end{array}$ & $24(5.2)$ & $76 \%$ & $\begin{array}{l}\text { Dis, WB, } \\
\text { Mindf, Real } \\
\text { func }\end{array}$ & MBSR (3) & No intervention (1) & $\begin{array}{l}\text { Post-int, } 2 \text { y, } 4 \\
y, 6 y\end{array}$ \\
\hline $\begin{array}{l}\text { Duncan } 2017 \\
\text { [129], US }\end{array}$ & 29 & $\begin{array}{l}\text { First-time pregnant } \\
\text { mothers (2) }\end{array}$ & NA & $100 \%$ & $\begin{array}{l}\text { Anx, Dep, Dis, } \\
\text { Mindf, Somat, } \\
\text { Self }\end{array}$ & Mind in Labour (6) & $\begin{array}{l}\text { Standard childbirth } \\
\text { education }(2)\end{array}$ & Post-int \\
\hline $\begin{array}{l}\text { Dvorakova } 2017 \\
\text { [130], US }\end{array}$ & 109 & $\begin{array}{l}\text { First year } \\
\text { undergraduates (1) }\end{array}$ & $18(0.4)$ & $66 \%$ & $\begin{array}{l}\text { Anx, Dep, WB, } \\
\text { Mindf, Somat, } \\
\text { Self }\end{array}$ & Learning to BREATHE (1) & Waitlist (1) & Post-int \\
\hline $\begin{array}{l}\text { Dykens } 2014 \\
\text { [131], US }\end{array}$ & 243 & $\begin{array}{l}\text { Mothers of disabled } \\
\text { children (2) }\end{array}$ & $41(8.9)$ & $100 \%$ & $\begin{array}{l}\text { Anx, Dep, Dis, } \\
\text { WB, Somat }\end{array}$ & MBSR (3) & $\begin{array}{l}\text { Positive Adult } \\
\text { Development (3) }\end{array}$ & $\begin{array}{l}\text { Post-int, } 1 \mathrm{~m} \text {, } \\
3 \mathrm{~m}, 6 \mathrm{~m}\end{array}$ \\
\hline $\begin{array}{l}\text { Dziok } 2010 \text { [132], } \\
\text { US }\end{array}$ & 52 & Local area adults (1) & $44(13.4)$ & $85 \%$ & Anx, Dep & Mindfulness meditation (3) & Waitlist (1) & $0-6 \mathrm{~m}$ \\
\hline $\begin{array}{l}\text { Esch } 2017 \text { [133], } \\
\text { Germany }\end{array}$ & 32 & Adults (1) & $27(7.6)$ & $67 \%$ & Cog, Mindf & $\begin{array}{l}\text { Combined breathing/ } \\
\text { mindfulness meditation } \\
\text { technique (1) }\end{array}$ & No intervention (1) & Post-int \\
\hline $\begin{array}{l}\text { Ferraioli } 2013 \\
\text { [134], US }\end{array}$ & 21 & $\begin{array}{l}\text { Parents of disabled } \\
\text { children (2) }\end{array}$ & NA & $67 \%$ & Dis, Mindf & $\begin{array}{l}\text { Mindfulness-Based Parent } \\
\text { Training (2) }\end{array}$ & $\begin{array}{l}\text { Skill-Based Parent } \\
\text { Training (2) }\end{array}$ & Post-int, $3 \mathrm{~m}$ \\
\hline $\begin{array}{l}\text { Fiocco } 2018 \text { [135], } \\
\text { Canada }\end{array}$ & 96 & Older adults (1) & $69(4.7)$ & $73 \%$ & Dis & $\begin{array}{l}\text { Mindfulness-Based } \\
\text { Intervention (1) }\end{array}$ & $\begin{array}{l}\text { Reading and Relaxation } \\
\text { Program (3) }\end{array}$ & Post-int \\
\hline $\begin{array}{l}\text { Flook } 2013 \text { [136], } \\
\text { US }\end{array}$ & 18 & $\begin{array}{l}\text { Public elementary } \\
\text { school teachers (2) }\end{array}$ & $43(9.9)$ & $89 \%$ & $\begin{array}{l}\text { Cog, Dis, } \\
\text { Mindf, Real } \\
\text { func, Self }\end{array}$ & MBSR (3) & Waitlist (1) & Post-int \\
\hline $\begin{array}{l}\text { Frisvold } 2009 \\
\text { [137], US }\end{array}$ & 40 & $\begin{array}{l}\text { Stressed midlife } \\
\text { female nurses (3) }\end{array}$ & $48(5.6)$ & $100 \%$ & $\begin{array}{l}\text { Dep, Dis, } \\
\text { Mindf, Somat }\end{array}$ & $\operatorname{MBSR}(3)$ & $\begin{array}{l}\text { Attention control } \\
\text { menopausal education (2) }\end{array}$ & Post-int, $2 \mathrm{~m}$ \\
\hline $\begin{array}{l}\text { Galante } 2018 \\
{[138,139], \text { UK }}\end{array}$ & 670 & $\begin{array}{l}\text { University students } \\
\text { (1) }\end{array}$ & $\begin{array}{l}\text { Median } \\
22, \text { range } \\
18-53\end{array}$ & $63 \%$ & $\begin{array}{l}\text { Dis, WB, Real } \\
\text { func }\end{array}$ & $\begin{array}{l}\text { Mindfulness Skills for } \\
\text { Students (1) }\end{array}$ & $\begin{array}{l}\text { No intervention/waitlist } \\
\text { (support as usual) (1) }\end{array}$ & $\begin{array}{l}\text { Post-int, } 1-4 \\
\mathrm{~m}, 10 \mathrm{~m}\end{array}$ \\
\hline $\begin{array}{l}\text { Gallego } 2014 \\
\text { [140], Spain }\end{array}$ & 125 & $\begin{array}{l}\text { University students } \\
\text { (1) }\end{array}$ & $20(3.7)$ & $58 \%$ & Dis & Mindfulness group (2) & $\begin{array}{l}\text { a) No intervention (1) b) } \\
\text { Physical Education ( } 3 \text { ) }\end{array}$ & Post-int \\
\hline
\end{tabular}


Table 1. (Continued)

\begin{tabular}{|c|c|c|c|c|c|c|c|c|}
\hline $\begin{array}{l}\text { First Author, } \\
\text { Year, Country }\end{array}$ & $N$ & $\begin{array}{l}\text { Participants } \\
\text { (category) }\end{array}$ & $\begin{array}{l}\text { Mean Age } \\
\text { (SD) }\end{array}$ & Women & Outcomes & Intervention (category) & Control/s (category) & $\begin{array}{l}\text { Outcome Time } \\
\text { Points }\end{array}$ \\
\hline $\begin{array}{l}\text { Gambrel } 2015 \\
\text { [141,142], US }\end{array}$ & 72 & Pregnant couples (2) & 32 & $52 \%$ & $\begin{array}{l}\text { Dis, WB, } \\
\text { Mindf }\end{array}$ & $\begin{array}{l}\text { Mindful Transition to } \\
\text { Parenthood Program (2) }\end{array}$ & Waitlist (1) & Post-int \\
\hline $\begin{array}{l}\text { Giannandrea } 2018 \\
\text { [143], Italy }\end{array}$ & 60 & Adults (1) & $36(12.1)$ & $67 \%$ & Cog, Mindf & $\operatorname{MBSR}(3)$ & Waitlist (1) & Post-int \\
\hline $\begin{array}{l}\text { Glass } 2019[144] \text {, } \\
\text { US }\end{array}$ & 57 & University athletes (1) & $19(1.3)$ & $85 \%$ & $\begin{array}{l}\text { Anx, Dep, Dis, } \\
\text { WB, Mindf }\end{array}$ & $\begin{array}{l}\text { Mindful Sport Performance } \\
\text { Enhancement (3) }\end{array}$ & Waitlist (1) & Post-int \\
\hline $\begin{array}{l}\text { Grandpierre } 2013 \\
\text { [145], Canada }\end{array}$ & 40 & $\begin{array}{l}\text { University students } \\
\text { with academic } \\
\text { difficulties (2) }\end{array}$ & $25(6.1)$ & $70 \%$ & $\begin{array}{l}\text { Cog, Dis, Real } \\
\text { func }\end{array}$ & $\begin{array}{l}\text { Mindfulness for Academic } \\
\text { Success (2) }\end{array}$ & Waitlist (1) & Post-int \\
\hline $\begin{array}{l}\text { Greenberg } 2010 \\
\text { [146,147], Israel }\end{array}$ & 76 & General adults (1) & $26(2.5)$ & $63 \%$ & $\operatorname{Cog}$ & Mindfulness training (2) & Waitlist (1) & Post-int \\
\hline $\begin{array}{l}\text { Greeson } 2014 \\
\text { [148], US }\end{array}$ & 90 & $\begin{array}{l}\text { University students } \\
\text { (1) }\end{array}$ & $25(5.7)$ & $66 \%$ & $\begin{array}{l}\text { Dis, Mindf, } \\
\text { Somat, Self }\end{array}$ & Koru (1) & Waitlist (1) & Post-int \\
\hline $\begin{array}{l}\text { Guardino } 2014 \\
\text { [149], US }\end{array}$ & 47 & $\begin{array}{l}\text { Stressed pregnant } \\
\text { women (3) }\end{array}$ & $33(4.8)$ & $100 \%$ & Dis, Mindf & MAPs (1) & Reading control group (2) & Post-int, $1.5 \mathrm{~m}$ \\
\hline $\begin{array}{l}\text { Haarig } 2016[150], \\
\text { Germany }\end{array}$ & 28 & Adults (1) & $42(11.8)$ & $70 \%$ & $\begin{array}{l}\text { Dep, Mindf, } \\
\text { Real func }\end{array}$ & $\begin{array}{l}\text { Mindfulness-Oriented Stress } \\
\text { Management Training (1) }\end{array}$ & Waitlist (1) & Post-int, $3 \mathrm{~m}$ \\
\hline $\begin{array}{l}\text { Hou } 2013 \\
{[151,152] \text {, China }}\end{array}$ & 141 & Caregivers (2) & $58(8.8)$ & $83 \%$ & $\begin{array}{l}\text { Anx, Dep, Dis, } \\
\text { Mindf, Self }\end{array}$ & MBSR (3) & $\begin{array}{l}\text { Self-help health education } \\
\text { (2) }\end{array}$ & Post-int, $3 \mathrm{~m}$ \\
\hline $\begin{array}{l}\text { Huang } 2015 \text { [153], } \\
\text { Taiwan }\end{array}$ & 144 & Employees (1) & 43 & $41 \%$ & Dis & $\begin{array}{l}\text { Mindfulness-based } \\
\text { intervention (3) }\end{array}$ & Waitlist (1) & Post-int, $2 \mathrm{~m}$ \\
\hline $\begin{array}{l}\text { Hunt } 2018[154] \\
\text { US }\end{array}$ & 119 & $\begin{array}{l}\text { Psychology } \\
\text { undergraduates (1) }\end{array}$ & 19 & $74 \%$ & Dep, Dis, WB & $\begin{array}{l}\text { a) Mindfulness training } \\
\text { merged with b) } \\
\text { Multicomponent } \\
\text { Mindfulness \& Yoga (1) }\end{array}$ & $\begin{array}{l}\text { a) No intervention (1) b) } \\
\text { Yoga Alone (3) merged } \\
\text { with c) Study Break with a } \\
\text { Therapy Dog (3) }\end{array}$ & Post-int \\
\hline $\begin{array}{l}\text { Hwang } 2019 \\
\text { [155], Australia }\end{array}$ & 185 & School teachers (2) & $42(12.6)$ & $84 \%$ & $\begin{array}{l}\text { Dis, Mindf, } \\
\text { Somat, Real } \\
\text { func, Self }\end{array}$ & Reconnected (3) & Waitlist (1) & Post-int, $1.5 \mathrm{~m}$ \\
\hline $\begin{array}{l}\text { Ireland } 2017 \\
\text { [156], Australia }\end{array}$ & 44 & Intern doctors (2) & $27(4.8)$ & $64 \%$ & Dis, Real func & $\begin{array}{l}\text { Mix mindfulness education } \\
\text { and practice (2) }\end{array}$ & $\begin{array}{l}\text { 1-hour break per week } \\
\text { (2) }\end{array}$ & Post-int \\
\hline $\begin{array}{l}\text { Isbel } 2019 \text { [157- } \\
\text { 159], Australia }\end{array}$ & 120 & Older adults (1) & 71 & $65 \%$ & Dis, Cog, WB & $\begin{array}{l}\text { Mindfulness-Based } \\
\text { Attention Training Program } \\
\text { (1) }\end{array}$ & $\begin{array}{l}\text { Computer-Based } \\
\text { Attention Training } \\
\text { Program (3) }\end{array}$ & Post-int \\
\hline $\begin{array}{l}\text { Jain } 2007 \text { [160], } \\
\text { US }\end{array}$ & 104 & $\begin{array}{l}\text { Healthcare students } \\
\text { (1) }\end{array}$ & 25 & $81 \%$ & Dis, WB & Mindfulness meditation (3) & $\begin{array}{l}\text { a) Waitlist (1) b) Somatic } \\
\text { Relaxation (3) }\end{array}$ & Post-int \\
\hline $\begin{array}{l}\text { James } 2018 \text { [161], } \\
\text { UK }\end{array}$ & 65 & $\begin{array}{l}\text { Students dealing with } \\
\text { perfectionism (3) }\end{array}$ & $\begin{array}{l}\text { Range 18- } \\
39\end{array}$ & $82 \%$ & $\begin{array}{l}\text { Anx, Dep, Dis, } \\
\text { Mindf, Self }\end{array}$ & MBCT (2) & $\begin{array}{l}\text { Pure Self-Help } \\
\text { Intervention (2) }\end{array}$ & Post-int, $2.5 \mathrm{~m}$ \\
\hline $\begin{array}{l}\text { Josefsson } 2014 \\
\text { [162], Sweden }\end{array}$ & 98 & Workers (1) & $50(10.3)$ & $91 \%$ & $\begin{array}{l}\text { Anx, Cog, Dep, } \\
\text { WB, Mindf }\end{array}$ & Mindfulness meditation (1) & $\begin{array}{l}\text { Relaxation Training } \\
\text { Condition (3) }\end{array}$ & Post-int \\
\hline $\begin{array}{l}\text { Kang } 2009 \text { [163], } \\
\text { South Korea }\end{array}$ & 41 & $\begin{array}{l}\text { Female nursing } \\
\text { students (1) }\end{array}$ & $23(1.2)$ & $100 \%$ & Dep, Dis & Stress coping program (3) & No intervention (1) & Post-int \\
\hline $\begin{array}{l}\text { Kaviani } 2008 \\
\text { [164,165], Iran }\end{array}$ & 30 & $\begin{array}{l}\text { Stressed female } \\
\text { students (3) }\end{array}$ & 21.5 & $100 \%$ & Anx, Dep & MBCT (2) & Waitlist (1) & $\begin{array}{l}\text { Post-int, } 1 \mathrm{~m}, \\
6 \mathrm{~m}\end{array}$ \\
\hline $\begin{array}{l}\text { Kingston } 2007 \\
\text { [166], UK }\end{array}$ & 45 & Students (1) & 23 & $79 \%$ & $\begin{array}{l}\text { Dis, WB, } \\
\text { Mindf }\end{array}$ & Mindfulness training (1) & Guided visual imagery (3) & Post-int \\
\hline $\begin{array}{l}\text { Kirk } 2016 \\
{[167,168], \text { US }}\end{array}$ & 51 & $\begin{array}{l}\text { University staff and } \\
\text { students (1) }\end{array}$ & $32(10)$ & $55 \%$ & $\begin{array}{l}\text { Dis, WB, } \\
\text { Mindf }\end{array}$ & Mindfulness training (1) & $\begin{array}{l}\text { Progressive muscle } \\
\text { relaxation (3) }\end{array}$ & Post-int \\
\hline $\begin{array}{l}\text { Klatt } 2009[46], \\
\text { US }\end{array}$ & 48 & Workers (1) & $45(2.5)$ & $34 \%$ & $\begin{array}{l}\text { Dis, Mindf, } \\
\text { Somat }\end{array}$ & MBSR (3) & Waitlist (1) & Post-int \\
\hline $\begin{array}{l}\text { Klatt } 2016 \\
{[169,170]} \\
\text { Denmark }\end{array}$ & 81 & Employees (1) & $43(9.3)$ & $69 \%$ & $\begin{array}{l}\text { Dis, Somat, } \\
\text { Real func }\end{array}$ & Mindfulness in Motion (3) & Waitlist (1) & Post-int \\
\hline
\end{tabular}


Table 1. (Continued)

\begin{tabular}{|c|c|c|c|c|c|c|c|c|}
\hline $\begin{array}{l}\text { First Author, } \\
\text { Year, Country }\end{array}$ & $N$ & $\begin{array}{l}\text { Participants } \\
\text { (category) }\end{array}$ & $\begin{array}{l}\text { Mean Age } \\
\text { (SD) }\end{array}$ & Women & Outcomes & Intervention (category) & Control/s (category) & $\begin{array}{l}\text { Outcome Time } \\
\text { Points }\end{array}$ \\
\hline $\begin{array}{l}\text { Kor } 2019 \text { [171], } \\
\text { China (HK) }\end{array}$ & 36 & Family caregivers (2) & $57(10.6)$ & $83 \%$ & $\begin{array}{l}\text { Anx, Dep, Dis, } \\
\text { Real func }\end{array}$ & Modified MBCT (2) & $\begin{array}{l}\text { No intervention (support } \\
\text { as usual) (1) }\end{array}$ & Post-int, $3 \mathrm{~m}$ \\
\hline $\begin{array}{l}\text { Krick } 2019 \text { [172], } \\
\text { Germany }\end{array}$ & 267 & Police officers (2) & $26(5.6)$ & $21 \%$ & Dis, Mindf, Self & $\begin{array}{l}\text { Mindfulness and Resource- } \\
\text { Based Worksite Training (2) }\end{array}$ & $\begin{array}{l}\text { No intervention (regular } \\
\text { education courses) (1) }\end{array}$ & Post-int \\
\hline $\begin{array}{l}\text { Kuhlmann } 2016 \\
{[173,174]} \\
\text { Germany }\end{array}$ & 182 & $\begin{array}{l}\text { Medical and dental } \\
\text { students (1) }\end{array}$ & $23(3.9)$ & $84 \%$ & $\begin{array}{l}\text { Dis, WB, } \\
\text { Mindf, Self }\end{array}$ & MediMind (2) & $\begin{array}{l}\text { a) Waitlist (1) b) } \\
\text { Autogenic training ( } 3 \text { ) }\end{array}$ & Post-int, $12 \mathrm{~m}$ \\
\hline $\begin{array}{l}\text { Lacerda } 2018 \\
\text { [175], Brazil } \\
\end{array}$ & 77 & Stressed workers (3) & 37 & $57 \%$ & $\begin{array}{l}\text { Anx, Cog, Dep, } \\
\text { Dis, Mindf }\end{array}$ & PROGRESS (1) & Waitlist (1) & Post-int \\
\hline $\begin{array}{l}\text { Lara-Cinisomo } \\
2019[176,177], \\
\text { US }\end{array}$ & 23 & Carers of veterans (2) & $58(12.4)$ & $96 \%$ & $\begin{array}{l}\text { Anx, Dep, Dis, } \\
\text { Mindf }\end{array}$ & MBCT (2) & Waitlist (1) & Post-int \\
\hline $\begin{array}{l}\text { Lebares } 2019 \\
{[178,179], \text { US }}\end{array}$ & 21 & Surgery interns (2) & $28(2.4)$ & $38 \%$ & $\begin{array}{l}\text { Cog, Dep, Dis, } \\
\text { Mindf, Real } \\
\text { func }\end{array}$ & MBSR (3) & Active control group (3) & Post-int, $10 \mathrm{~m}$ \\
\hline $\begin{array}{l}\text { Lee } 2010 \text { [180], } \\
\text { South Korea }\end{array}$ & 75 & $\begin{array}{l}\text { Middle-aged women } \\
\text { (1) }\end{array}$ & $41(5.8)$ & $100 \%$ & $\begin{array}{l}\text { Anx, Dep, WB, } \\
\text { Mindf, Somat, } \\
\text { Self }\end{array}$ & $\begin{array}{l}\text { MBCT + self-compassion } \\
(2)\end{array}$ & Waitlist (1) & Post-int \\
\hline $\begin{array}{l}\text { Li } 2018[181], \\
\text { China }\end{array}$ & 34 & General adults (1) & $29(9.2)$ & $67 \%$ & Cog, Mindf & MBCT (2) & Waitlist (1) & Post-int \\
\hline $\begin{array}{l}\text { Lin } 2019 \text { [182], } \\
\text { China }\end{array}$ & 110 & Nurses (2) & $32(6.9)$ & $93 \%$ & $\begin{array}{l}\text { Dis, WB, Real } \\
\text { func }\end{array}$ & MBSR (3) & Waitlist (1) & Post-int, $3 \mathrm{~m}$ \\
\hline $\begin{array}{l}\text { Liu } 2013 \text { [183], } \\
\text { China }\end{array}$ & 72 & $\begin{array}{l}\text { College and graduate } \\
\text { students (1) }\end{array}$ & $29(13.4)$ & $89 \%$ & $\begin{array}{l}\text { Dis, WB, } \\
\text { Mindf }\end{array}$ & Mindfulness training (2) & Waitlist (1) & Post-int \\
\hline $\begin{array}{l}\text { Liu } 2015 \text { [184], } \\
\text { China }\end{array}$ & 65 & General adults (1) & $27(6.7)$ & $70 \%$ & Mindf & MBCT (2) & Waitlist (1) & Post-int \\
\hline $\begin{array}{l}\text { Lo } 2017 \text { [185], } \\
\text { China }\end{array}$ & 180 & $\begin{array}{l}\text { Parents of disabled } \\
\text { children (2) }\end{array}$ & $39(5.9)$ & $94 \%$ & $\begin{array}{l}\text { Dep, Dis, } \\
\text { Mindf }\end{array}$ & $\begin{array}{l}\text { Brief Mindfulness-Based } \\
\text { Program (2) }\end{array}$ & Waitlist (1) & Post-int \\
\hline $\begin{array}{l}\text { Lonnberg } 2020 \\
{[186,187],} \\
\text { Sweden }\end{array}$ & 193 & Pregnant women (2) & 32 & $100 \%$ & $\begin{array}{l}\text { Dep, Dis, } \\
\text { Mindf }\end{array}$ & $\begin{array}{l}\text { Mindfulness-Based } \\
\text { Childbirth \& Parenting (1) }\end{array}$ & Lamaze program (3) & $\begin{array}{l}\text { Post-int, 3-5 } \\
\mathrm{m}\end{array}$ \\
\hline $\begin{array}{l}\text { Lopez-Maya } 2019 \\
\text { [188], US }\end{array}$ & 76 & Stressed adults (3) & $43(15.3)$ & $79 \%$ & $\begin{array}{l}\text { Dep, Dis, } \\
\text { Mindf, Self }\end{array}$ & MAPs (1) & $\begin{array}{l}\text { Health Education } \\
\text { Program (3) }\end{array}$ & Post-int \\
\hline $\begin{array}{l}\text { Lynch } 2018 \text { [189], } \\
\text { UK }\end{array}$ & 38 & $\begin{array}{l}\text { University students } \\
\text { (1) }\end{array}$ & $26(8.3)$ & $77 \%$ & $\begin{array}{l}\text { Anx, Dep, Dis, } \\
\text { Mindf }\end{array}$ & $\begin{array}{l}\text { Mindfulness-Based Coping } \\
\text { with University Life (6) }\end{array}$ & Waitlist (1) & Post-int \\
\hline $\begin{array}{l}\text { Ma } 2019 \text { [190], } \\
\text { China (HK) }\end{array}$ & 43 & Stressed adults (3) & $39(15.1)$ & $83 \%$ & $\begin{array}{l}\text { Anx, Cog, Dep, } \\
\text { Dis, Mindf }\end{array}$ & MBCT (2) & $\begin{array}{l}\text { Physical exercise program } \\
\text { (3) }\end{array}$ & Post-int, $2 \mathrm{~m}$ \\
\hline $\begin{array}{l}\text { MacCoon } 2012 \\
\text { [191-194], US }\end{array}$ & 63 & General adults (1) & $48(10.7)$ & $63 \%$ & $\begin{array}{l}\text { Cog, Dis, WB, } \\
\text { Mindf, Somat }\end{array}$ & $\operatorname{MBSR}(3)$ & $\begin{array}{l}\text { a) Health Enhancement } \\
\text { Program (3) b) Waitlist } \\
\text { (1) }\end{array}$ & Post-int, $4 \mathrm{~m}$ \\
\hline $\begin{array}{l}\text { Malarkey } 2013 \\
{[169,195], \text { US }}\end{array}$ & 186 & University workers (1) & 50 & $88 \%$ & $\begin{array}{l}\text { Dep, Dis, } \\
\text { Somat }\end{array}$ & $\begin{array}{l}\text { Mindfulness-based } \\
\text { intervention (3) }\end{array}$ & $\begin{array}{l}\text { Lifestyle education group } \\
\text { (2) }\end{array}$ & Post-int \\
\hline $\begin{array}{l}\text { Malinowski } 2017 \\
\text { [196,197], UK }\end{array}$ & 56 & Older adults (1) & 65 & $73 \%$ & $\begin{array}{l}\text { Cog, WB, } \\
\text { Mindf, Self }\end{array}$ & Mindfulness training (2) & Brain training group (3) & Post-int \\
\hline $\begin{array}{l}\text { Manotas } 2014 \\
{[198,199]} \\
\text { Colombia }\end{array}$ & 131 & $\begin{array}{l}\text { Healthcare workers } \\
\text { (2) }\end{array}$ & $39(8.2)$ & $90 \%$ & Dis, Mindf & Mindfulness training (3) & Waitlist (1) & Post-int \\
\hline $\begin{array}{l}\text { Moody } 2013 \\
\text { [200], US and } \\
\text { Israel }\end{array}$ & 47 & $\begin{array}{l}\text { Paediatric oncology } \\
\text { staff }(2)\end{array}$ & NA & $80 \%$ & $\begin{array}{l}\text { Dep, Dis, Real } \\
\text { func }\end{array}$ & $\begin{array}{l}\text { Mindfulness-based course } \\
\text { (3) }\end{array}$ & No intervention (1) & Post-int \\
\hline $\begin{array}{l}\text { Moritz } 2006 \text { [201], } \\
\text { Canada }\end{array}$ & 165 & $\begin{array}{l}\text { Stressed individuals } \\
\text { (3) }\end{array}$ & 44 & $78 \%$ & Dis & Meditation group (3) & $\begin{array}{l}\text { a) Waitlist (1) b) } \\
\text { Spirituality teaching } \\
\text { program (3) }\end{array}$ & Post-int, $1 \mathrm{~m}$ \\
\hline
\end{tabular}


Table 1. (Continued)

\begin{tabular}{|c|c|c|c|c|c|c|c|c|}
\hline $\begin{array}{l}\text { First Author, } \\
\text { Year, Country }\end{array}$ & $N$ & $\begin{array}{l}\text { Participants } \\
\text { (category) }\end{array}$ & $\begin{array}{l}\text { Mean Age } \\
\text { (SD) }\end{array}$ & Women & Outcomes & Intervention (category) & Control/s (category) & $\begin{array}{l}\text { Outcome Time } \\
\text { Points }\end{array}$ \\
\hline $\begin{array}{l}\text { Moynihan } 2013 \\
\text { [202-208], US }\end{array}$ & 219 & Older adults (1) & $74(6.7)$ & $62 \%$ & $\begin{array}{l}\text { Cog, Dep, Dis, } \\
\text { WB, Mindf, } \\
\text { Somat }\end{array}$ & MBSR (3) & Waitlist (1) & Post-int, $6 \mathrm{~m}$ \\
\hline $\begin{array}{l}\text { Mrazek } 2013 \\
\text { [209], US }\end{array}$ & 48 & $\begin{array}{l}\text { Undergraduate } \\
\text { students (1) }\end{array}$ & $21(2.1)$ & $71 \%$ & Cog, Real func & Mindfulness class (1) & Nutrition class (2) & Post-int \\
\hline $\begin{array}{l}\text { Neece } 2014 \text { [210- } \\
212], \text { US }\end{array}$ & 130 & $\begin{array}{l}\text { Parents of disabled } \\
\text { children (2) }\end{array}$ & $36(7.6)$ & $96 \%$ & Dep, Dis, WB & MBSR (3) & Waitlist (1) & Post-int \\
\hline $\begin{array}{l}\text { Norouzi } 2020 \\
\text { [213], Iran }\end{array}$ & 40 & Retired athletes (1) & $34(1.7)$ & $0 \%$ & $\begin{array}{l}\text { Anx, Dep, Dis, } \\
\text { WB }\end{array}$ & MBSR (3) & $\begin{array}{l}\text { Active control condition } \\
(2)\end{array}$ & Post-int, $1 \mathrm{~m}$ \\
\hline $\begin{array}{l}\text { Nyklicek } 2008 \\
\text { [214], the } \\
\text { Netherlands }\end{array}$ & 60 & Distressed adults (3) & $46(9.9)$ & $67 \%$ & Dis, WB & MBSR (3) & Waitlist (1) & Post-int \\
\hline $\begin{array}{l}\text { O’Donnell } 2017 \\
\text { [215,216], US }\end{array}$ & 29 & Caregivers (2) & $71(6.7)$ & $93 \%$ & $\begin{array}{l}\text { Dep, Dis, } \\
\text { Mindf, Somat, } \\
\text { Real func, Self }\end{array}$ & MBSR (3) & $\begin{array}{l}\text { Progressive Muscle } \\
\text { Relaxation (3) }\end{array}$ & $\begin{array}{l}\text { Post-int, } 2 \mathrm{~m} \text {, } \\
6 \mathrm{~m}, \sim 12 \mathrm{~m}\end{array}$ \\
\hline $\begin{array}{l}\text { Oken } 2010 \text { [217], } \\
\text { US }\end{array}$ & 31 & $\begin{array}{l}\text { Caregivers of relatives } \\
\text { with dementia ( } 2 \text { ) }\end{array}$ & $65(9.3)$ & $81 \%$ & $\begin{array}{l}\text { Cog, Dep, Dis, } \\
\text { Mindf, Somat, } \\
\text { Real func, Self }\end{array}$ & Mindfulness meditation (2) & $\begin{array}{l}\text { a) Respite care only (1) b) } \\
\text { Dementia education class } \\
\text { (3) }\end{array}$ & Post-int \\
\hline $\begin{array}{l}\text { Pan } 2018 \\
{[218,219] \text {, Taiwan }}\end{array}$ & 104 & Pregnant women (2) & $33(3.8)$ & $100 \%$ & $\begin{array}{l}\text { Dep, Mindf, } \\
\text { Self }\end{array}$ & $\begin{array}{l}\text { Mindfulness-Based } \\
\text { Childbirth and Parenting (2) }\end{array}$ & $\begin{array}{l}\text { Conventional childbirth } \\
\text { education ( } 3 \text { ) }\end{array}$ & $\begin{array}{l}\text { Post-int, } 36- \\
\text { week gestation, } \\
3 \mathrm{~m} \text { after birth } \\
\end{array}$ \\
\hline $\begin{array}{l}\text { Park } 2016[220], \\
\text { South Korea }\end{array}$ & 60 & $\begin{array}{l}\text { Middle-aged women } \\
(1)\end{array}$ & $54(5.4)$ & $100 \%$ & $\begin{array}{l}\text { Dep, Dis, } \\
\text { Somat }\end{array}$ & Korean MBSR (3) & Waitlist (1) & Post-int \\
\hline $\begin{array}{l}\text { Perez-Blasco } 2013 \\
\text { [221], Spain }\end{array}$ & 26 & $\begin{array}{l}\text { Breastfeeding mothers } \\
(2)\end{array}$ & $34(4.7)$ & $100 \%$ & $\begin{array}{l}\text { Dis, WB, } \\
\text { Mindf, Self }\end{array}$ & Mindfulness training (2) & Waitlist (1) & Post-int \\
\hline $\begin{array}{l}\text { Perez-Blasco } 2016 \\
\text { [222], Spain }\end{array}$ & 45 & Older adults (1) & $64(4.1)$ & $67 \%$ & $\begin{array}{l}\text { Anx, Dep, Dis, } \\
\text { Real func }\end{array}$ & Mindfulness training (2) & Waitlist (1) & Post-int \\
\hline $\begin{array}{l}\text { Phang } 2015 \text { [223], } \\
\text { Malaysia }\end{array}$ & 75 & medical students (1) & $21(1.1)$ & $76 \%$ & Dis, Mindf, Self & Mindful-Gym (3) & Waitlist (1) & Post-int, $6 \mathrm{~m}$ \\
\hline $\begin{array}{l}\text { Pipe } 2009 \text { [224], } \\
\text { US }\end{array}$ & 33 & Nurse leaders (2) & $50(6.8)$ & $97 \%$ & Dis, Self & Mindfulness meditation (1) & $\begin{array}{l}\text { Structured educational } \\
\text { series (3) }\end{array}$ & Post-int \\
\hline $\begin{array}{l}\text { Plummer } 2018 \\
{[225,226], \text { US }}\end{array}$ & 105 & Nursing students (1) & 23 & $93 \%$ & Dis, Mindf & $\begin{array}{l}\text { Mindfulness-Centred Stress } \\
\text { Reduction (1) }\end{array}$ & No intervention (1) & Post-int, $3 \mathrm{~m}$ \\
\hline $\begin{array}{l}\text { Pots } 2014 \text { [227], } \\
\text { the Netherlands }\end{array}$ & 151 & $\begin{array}{l}\text { Adults with depressive } \\
\text { symptoms (3) }\end{array}$ & $48(11.3)$ & $78 \%$ & $\begin{array}{l}\text { Anx, Dis, WB, } \\
\text { Mindf }\end{array}$ & MBCT (2) & Waitlist (1) & Post-int \\
\hline $\begin{array}{l}\text { Prakash } 2015 \\
{[228,229], \text { US }}\end{array}$ & 74 & Older adults (1) & $66(4)$ & $58 \%$ & Cog, Mindf & $\begin{array}{l}\text { Mindfulness-Based } \\
\text { Attention Training (3) }\end{array}$ & Lifestyle education (2) & Post-int \\
\hline $\begin{array}{l}\text { Richards } 2012 \\
{[230,231], \text { US }}\end{array}$ & 47 & Undergraduates (1) & $21(7.5)$ & $85 \%$ & Mindf, Self & $\begin{array}{l}\text { Brief mindfulness } \\
\text { intervention and LKM } \\
\text { exercises (2) }\end{array}$ & Waitlist (1) & Post-int \\
\hline $\begin{array}{l}\text { Richards } 2013 \\
\text { [232], US }\end{array}$ & 30 & Undergraduates (1) & $21(3.2)$ & $72 \%$ & Mindf, Self & $\begin{array}{l}\text { Brief mindfulness } \\
\text { intervention (1) }\end{array}$ & Waitlist (1) & Post-int \\
\hline $\begin{array}{l}\text { Robins } 2012 \\
{[233,234], \text { US }}\end{array}$ & 56 & Adults (1) & $46(13)$ & $84 \%$ & $\begin{array}{l}\text { Mindf, Real } \\
\text { func, Self }\end{array}$ & MBSR (3) & Waitlist (1) & Post-int \\
\hline $\begin{array}{l}\text { Roeser } 2013 \text { [235- } \\
\text { 237], US and } \\
\text { Canada }\end{array}$ & 113 & School teachers (2) & $47(9.2)$ & $89 \%$ & $\begin{array}{l}\text { Cog, Dep, } \\
\text { Mindf, Real } \\
\text { func }\end{array}$ & $\begin{array}{l}\text { Stress Management and } \\
\text { Relaxation Techniques in } \\
\text { Education (2) }\end{array}$ & Waitlist (1) & Post-int, $3 \mathrm{~m}$ \\
\hline $\begin{array}{l}\text { Sampl } 2017 \text { [238], } \\
\text { Australia }\end{array}$ & 109 & Undergraduates (1) & $22(4.6)$ & $78 \%$ & $\begin{array}{l}\text { Anx, Dis, } \\
\text { Mindf, Real } \\
\text { func }\end{array}$ & $\begin{array}{l}\text { Mindfulness-Based Self- } \\
\text { Leadership Training (2) }\end{array}$ & Waitlist (1) & $1-3 \mathrm{~m}$ \\
\hline $\begin{array}{l}\text { Schellekens } 2017 \\
\text { [239-241], the } \\
\text { Netherlands }\end{array}$ & 44 & $\begin{array}{l}\text { Lung cancer patient } \\
\text { partners ( } 2)\end{array}$ & $59(7.9)$ & $53 \%$ & Dis, Mindf, Self & MBSR (3) & Waitlist (1) & Post-int, $3 \mathrm{~m}$ \\
\hline
\end{tabular}


Table 1. (Continued)

\begin{tabular}{|c|c|c|c|c|c|c|c|c|}
\hline $\begin{array}{l}\text { First Author, } \\
\text { Year, Country }\end{array}$ & $N$ & $\begin{array}{l}\text { Participants } \\
\text { (category) }\end{array}$ & $\begin{array}{l}\text { Mean Age } \\
\text { (SD) }\end{array}$ & Women & Outcomes & Intervention (category) & Control/s (category) & $\begin{array}{l}\text { Outcome Time } \\
\text { Points }\end{array}$ \\
\hline $\begin{array}{l}\text { Schroeder } 2018 \\
\text { [242], US }\end{array}$ & 33 & $\begin{array}{l}\text { Primary care } \\
\text { physicians (2) }\end{array}$ & $43(8.4)$ & $73 \%$ & $\begin{array}{l}\text { Dis, Mindf, } \\
\text { Real func }\end{array}$ & $\begin{array}{l}\text { Mindful Medicine } \\
\text { Curriculum (2) }\end{array}$ & Waitlist (1) & Post-int, $3 \mathrm{~m}$ \\
\hline $\begin{array}{l}\text { Sevinc } 2018 \text { [243], } \\
\text { US }\end{array}$ & 50 & Adults (1) & $39(9.6)$ & $56 \%$ & $\begin{array}{l}\text { Anx, Dis, } \\
\text { Mindf, Self }\end{array}$ & MBSR (3) & Relaxation response (3) & Post-int \\
\hline $\begin{array}{l}\text { Shapiro } 1998 \\
\text { [244], US }\end{array}$ & 78 & Medical students (1) & NA & $56 \%$ & Anx, Dis & $\begin{array}{l}\text { Stress Reduction and } \\
\text { Relaxation Program (3) }\end{array}$ & Waitlist (1) & Post-int \\
\hline $\begin{array}{l}\text { Shapiro } 2005 \\
\text { [245], US }\end{array}$ & 38 & $\begin{array}{l}\text { Healthcare } \\
\text { professionals (2) }\end{array}$ & $\begin{array}{l}\text { Range } 18- \\
65\end{array}$ & NA & $\begin{array}{l}\text { Dis, WB, Real } \\
\text { func, Self }\end{array}$ & MBSR (3) & Waitlist (1) & Post-int \\
\hline $\begin{array}{l}\text { Shapiro } 2019 \\
\text { [246], US }\end{array}$ & 41 & Medical students (1) & 24 & $78 \%$ & $\begin{array}{l}\text { Dep, Dis, } \\
\text { Mindf }\end{array}$ & MBSR (3) & Waitlist (1) & Post-int \\
\hline $\begin{array}{l}\text { Shearer } 2016 \\
\text { [247], US }\end{array}$ & 74 & Undergraduates (1) & NA & $57 \%$ & Dep, Mindf & Mindfulness meditation (3) & $\begin{array}{l}\text { a) No intervention (1) b) } \\
\text { De-stress with dogs (3) }\end{array}$ & Post-int \\
\hline $\begin{array}{l}\text { Smart } 2017 \\
{[248,249], \text { Canada }}\end{array}$ & 38 & $\begin{array}{l}\text { Healthy older adults } \\
\text { (1) }\end{array}$ & $70(3.5)$ & $53 \%$ & Anx, Cog, Self & Wisdom Mind (3) & $\begin{array}{l}\text { Memory and Aging } \\
\text { Program (3) }\end{array}$ & Post-int \\
\hline $\begin{array}{l}\square \text { tefan } 2018 \\
\text { [250], Romania }\end{array}$ & 71 & Undergraduates (1) & $19(1)$ & $93 \%$ & $\begin{array}{l}\text { Anx, Dep, Dis, } \\
\text { Self }\end{array}$ & MBSR (2) & Waitlist (1) & Post-int \\
\hline $\begin{array}{l}\text { Steinberg } 2016 \\
\text { [251-254], US }\end{array}$ & 32 & $\begin{array}{l}\text { Intensive Care Unit } \\
\text { Personnel (2) }\end{array}$ & $40(11.3)$ & $88 \%$ & $\begin{array}{l}\text { Dis, Mindf, } \\
\text { Real func }\end{array}$ & Mindfulness in Motion (3) & Waitlist (1) & Post-int \\
\hline $\begin{array}{l}\text { Strub } 2013 \text { [255], } \\
\text { Luxembourg }\end{array}$ & 20 & Employees (1) & $\begin{array}{l}85 \%<45 \\
\text { years old }\end{array}$ & $40 \%$ & $\begin{array}{l}\text { Dep, Dis, Real } \\
\text { func }\end{array}$ & MBCT (2) & No intervention (1) & Post-int \\
\hline $\begin{array}{l}\text { Thomas } 2016 \\
\text { [256], United } \\
\text { Arab Emirates }\end{array}$ & 24 & $\begin{array}{l}\text { Psychology college } \\
\text { students (1) }\end{array}$ & $21(2.3)$ & $76 \%$ & Dep & MBSR (3) & Waitlist (1) & Post-int \\
\hline $\begin{array}{l}\text { Van Berkel } 2014 \\
\text { [257-262], the } \\
\text { Netherlands }\end{array}$ & 257 & Employees (1) & $46(9.5)$ & $87 \%$ & $\begin{array}{l}\text { Mindf, Real } \\
\text { func }\end{array}$ & $\begin{array}{l}\text { Mindful Vitality in Practice } \\
\text { (2) }\end{array}$ & No intervention (1) & Post-int, $6 \mathrm{~m}$ \\
\hline $\begin{array}{l}\text { Van Dam } 2014 \\
\text { [263], US }\end{array}$ & 56 & Stressed adults (3) & $40(14.4)$ & $61 \%$ & $\begin{array}{l}\text { Anx, Dep, Dis, } \\
\text { Mindf, Self }\end{array}$ & Mindfulness meditation (3) & Waitlist (1) & $1 \mathrm{~m}$ \\
\hline $\begin{array}{l}\text { Van Dijk } 2017 \\
\text { [264,265], the } \\
\text { Netherlands }\end{array}$ & 167 & $\begin{array}{l}\text { Medical } \\
\text { undergraduates (1) }\end{array}$ & $25(1.8)$ & $79 \%$ & $\begin{array}{l}\text { Dis, WB, } \\
\text { Mindf, Real } \\
\text { func }\end{array}$ & MBSR (3) & $\begin{array}{l}\text { No intervention } \\
\text { (clerkships as usual) (1) }\end{array}$ & $\begin{array}{l}\text { Post-int, } 4 \mathrm{~m} \text {, } \\
9 \mathrm{~m}, 12 \mathrm{~m}, 17 \\
\mathrm{~m}\end{array}$ \\
\hline $\begin{array}{l}\text { Verweij } 2018 \\
{[266,267], \text { the }} \\
\text { Netherlands }\end{array}$ & 148 & Medical doctors (2) & $31(4.6)$ & $88 \%$ & $\begin{array}{l}\text { WB, Mindf, } \\
\text { Real func, Self }\end{array}$ & MBSR (3) & Waitlist (1) & Post-int \\
\hline $\begin{array}{l}\text { Vieten } 2008 \text { [268], } \\
\text { US }\end{array}$ & 34 & $\begin{array}{l}\text { Pregnant women with } \\
\text { mood concerns ( } 3 \text { ) }\end{array}$ & $34(3.8)$ & $100 \%$ & $\begin{array}{l}\text { Dep, Dis, } \\
\text { Mindf }\end{array}$ & Mindful Motherhood (2) & Waitlist (1) & Post-int, $1 \mathrm{~m}$ \\
\hline $\begin{array}{l}\text { Vinesett } 2017 \\
\text { [269], US }\end{array}$ & 21 & Community adults (1) & $48(8.1)$ & $100 \%$ & $\begin{array}{l}\text { Dep, Dis, WB, } \\
\text { Real func }\end{array}$ & MBSR (3) & Ngoma ceremony (3) & Post-int, $1 \mathrm{~m}$ \\
\hline $\begin{array}{l}\text { Wang } 2012 \text { [270], } \\
\text { China }\end{array}$ & 31 & $\begin{array}{l}\text { University students } \\
\text { (1) }\end{array}$ & $\begin{array}{l}\text { Range 17- } \\
25\end{array}$ & $71 \%$ & $\operatorname{Cog}$ & Mindfulness (2) & Waitlist (1) & Post-int \\
\hline $\begin{array}{l}\text { Whitebird } 2013 \\
\text { [271,272], US }\end{array}$ & 78 & Carers (2) & $57(9.9)$ & $89 \%$ & $\begin{array}{l}\text { Dep, Dis, Real } \\
\text { func }\end{array}$ & MBSR (3) & $\begin{array}{l}\text { Standard community } \\
\text { caregiver education and } \\
\text { social support ( } 3 \text { ) }\end{array}$ & Post-int, $4 \mathrm{~m}$ \\
\hline $\begin{array}{l}\text { Williams } 2001 \\
\text { [273], US }\end{array}$ & 138 & Stressed adults (3) & $43(2.2)$ & $72 \%$ & Dis & MBSR (3) & $\begin{array}{l}\text { No intervention (standard } \\
\text { educational materials) (1) }\end{array}$ & Post-int, $3 \mathrm{~m}$ \\
\hline $\begin{array}{l}\text { Wilson } 2012 \\
\text { [274], US }\end{array}$ & 96 & Working adults (1) & $\begin{array}{l}\text { Range 23- } \\
64\end{array}$ & $66 \%$ & $\begin{array}{l}\text { Dis, WB, } \\
\text { Mindf, Somat, } \\
\text { Real func }\end{array}$ & $\begin{array}{l}\text { (a) MBAP merged with (b) } \\
\text { low dose MBSR (5) }\end{array}$ & No intervention (1) & Post-int, $1 \mathrm{~m}$ \\
\hline $\begin{array}{l}\text { Wong } 2018 \text { [275], } \\
\text { China (HK) }\end{array}$ & 197 & $\begin{array}{l}\text { Peri-/postmenopausal } \\
\text { women (2) }\end{array}$ & $52(3.1)$ & $100 \%$ & $\begin{array}{l}\text { Dis, Mindf, } \\
\text { Somat }\end{array}$ & MBSR (3) & $\begin{array}{l}\text { Menopause Education } \\
\text { Control (3) }\end{array}$ & $\begin{array}{l}\text { Post-int, } 3 \mathrm{~m} \text {, } \\
6 \mathrm{~m}\end{array}$ \\
\hline $\begin{array}{l}\text { Woolhouse } 2014 \\
\text { [276], Australia }\end{array}$ & 32 & Pregnant women (2) & $33(0.6)$ & $100 \%$ & $\begin{array}{l}\text { Anx, Dep, Dis, } \\
\text { Mindf }\end{array}$ & $\begin{array}{l}\text { MindBabyBody Programme } \\
\text { (2) }\end{array}$ & Care as usual (1) & Post-int \\
\hline
\end{tabular}


Table 1. (Continued)

\begin{tabular}{l|l|l|l|l|l|l|l|l}
\hline $\begin{array}{l}\text { First Author, } \\
\text { Year, Country }\end{array}$ & $\boldsymbol{N}$ & $\begin{array}{l}\text { Participants } \\
\text { (category) }\end{array}$ & $\begin{array}{l}\text { Mean Age } \\
\text { (SD) }\end{array}$ & Women & Outcomes & Intervention (category) & Control/s (category) & $\begin{array}{l}\text { Outcome Time } \\
\text { Points }\end{array}$ \\
\hline $\begin{array}{l}\text { Xu 2015 [277], } \\
\text { China }\end{array}$ & 90 & Adults (1) & $31(8)$ & $56 \%$ & Dis, Mindf & Mindfulness training (2) & Waitlist (1) \\
\hline $\begin{array}{l}\text { Yazdanimehr } \\
\begin{array}{l}\text { Iran [278,279], } \\
\text { Zhang 2018 [280], } \\
\text { China }\end{array}\end{array}$ & 80 & Pregnant women (2) & $26(5.2)$ & $100 \%$ & Anx, Dep, Dis & MiCBT (2) & $\begin{array}{l}\text { Usual prenatal care } \\
\text { services (1) }\end{array}$ & \begin{tabular}{l} 
Post-int, 1 m \\
\hline
\end{tabular}
\end{tabular}

Some studies did not report the mean age and/or its standard deviation. Participant categories according to intervention targeting: (1) Universal; (2) Selective; and (3) Indicated. Intervention categories: (1) no other components; (2) psychoeducation and/or nonmeditative psychological exercises; (3) physical exercises; (4) other types of meditation; (5) arts; and (6) other/unclear. Control/s categories: (1) passive; (2) nonspecific; and (3) specific. Review outcome abbreviations: Anx, Anxiety; Cog, Cognitive functioning; Dep, Depression; Dis, Distress; Mind, Mindfulness; Real func, Real life functioning; Self, Relationship with self; Somat, Psychosomatic outcomes; WB, Mental well-being. Intervention abbreviations: LKM, Loving-Kindness Meditation; MAPs; Mindful Awareness Practices; MBAP, Mindfulness-Based Art Processing; MBCT, Mindfulness-Based Cognitive Therapy; MBSR, Mindfulness-Based Stress Reduction; MiCBT, Mindfulness-Integrated Cognitive Behaviour Therapy. Control treatment abbreviations: CBT, Cognitive Behavioural Therapy. Time point abbreviations: m, Month/s of follow-up post-intervention; Post-int, PostIntervention; $\mathrm{y}$, Year/s of follow-up post-intervention.

95\% PI -1.19 to 0.06 ), depression (SMD $=-0.53 ; 95 \%$ CI -0.72 to $-0.34 ; p$-value $<0.001$; $95 \%$ PI -1.14 to 0.07 ), psychological distress (SMD $=-0.45 ; 95 \%$ CI -0.58 to $-0.31 ; p-$ value $<0.001 ; 95 \% \mathrm{PI}-1.04$ to 0.14 ), and mental well-being ( $\mathrm{SMD}=0.33$; $95 \% \mathrm{CI} 0.11$ to $0.54 ; p$-value $=0.003 ; 95 \%$ PI -0.29 to 0.94 ) (Fig 2, S1 Appendix). Effects, according to Cohen's rule of thumb [281], ranged from small (well-being) to moderate (distress, depression, anxiety). However, the prediction intervals indicated that in more than $5 \%$ of trial settings, MBPs may not improve anxiety and depression; indeed, in those settings, the outcome scores following MBPs may even be higher for distress and lower for well-being when compared to those following a passive control.

Very few studies compared MBPs with active nonspecific control groups, so results were interpreted with caution (Fig 2, S1 Appendix). On average, MBPs improved depression (SMD $=-0.46 ; 95 \% \mathrm{CI}-0.81$ to $-0.10 ; p$-value $=0.012 ; 95 \% \mathrm{PI}-1.57$ to 0.66 ) between 1 and 6 months post-intervention with a moderate effect size, although PIs did not rule out other directions of effect. Anxiety showed a trend towards improvement (SMD $=-0.47 ; 95 \% \mathrm{CI}$ -0.87 to $-0.08 ; p$-value $=0.019 ; 95 \%$ PI -1.60 to 0.66 ). There was no evidence to support MBPs improving distress (SMD $=-0.14 ; 95 \% \mathrm{CI}-0.51$ to 0.23 ; $p$-value $=0.47 ; 95 \% \mathrm{PI}-1.26$ to 0.98 ). Well-being showed improvement $(\mathrm{SMD}=1.40 ; 95 \% \mathrm{CI} 0.35$ to $2.46 ; p$-value $=0.009$; $95 \%$ PI -0.19 to 3.00 ), but only 1 study measured it, so although multivariate meta-analysis "borrows strength" from other outcomes and studies through their correlations, this result is unreliable [282].

Compared with active control interventions designed to deliver specific effects (Fig 2, S1 Appendix), there was no clear evidence that MBPs improved any primary outcome domain (For anxiety: $\mathrm{SMD}=0.07 ; 95 \% \mathrm{CI}-0.20$ to $0.35 ; p$-value $=0.61 ; 95 \% \mathrm{PI}-0.34$ to 0.48 . For depression: $\mathrm{SMD}=-0.17 ; 95 \% \mathrm{CI}-0.32$ to $-0.01 ; p-$ value $=0.04 ; 95 \%$ PI -0.50 to 0.16 . For distress: SMD $-0.01 ; 95 \% \mathrm{CI}-0.15$ to 0.13 ; $p$-value $=0.90 ; 95 \% \mathrm{PI}-0.33,0.32$. For well-being: $\mathrm{SMD}=0.03 ; 95 \% \mathrm{CI}-0.18,0.24 ; p$-value $=0.79 ; 95 \%$ PI $-0.33,0.39$ ). Too few studies measured anxiety or well-being outcomes for MBPs relative to active control interventions, so their results are unreliable.

Most studies $(121,89 \%)$ do not mention having measured adverse events or effects. Of those that did, 12 trials reported no adverse events or effects during the study. One study 


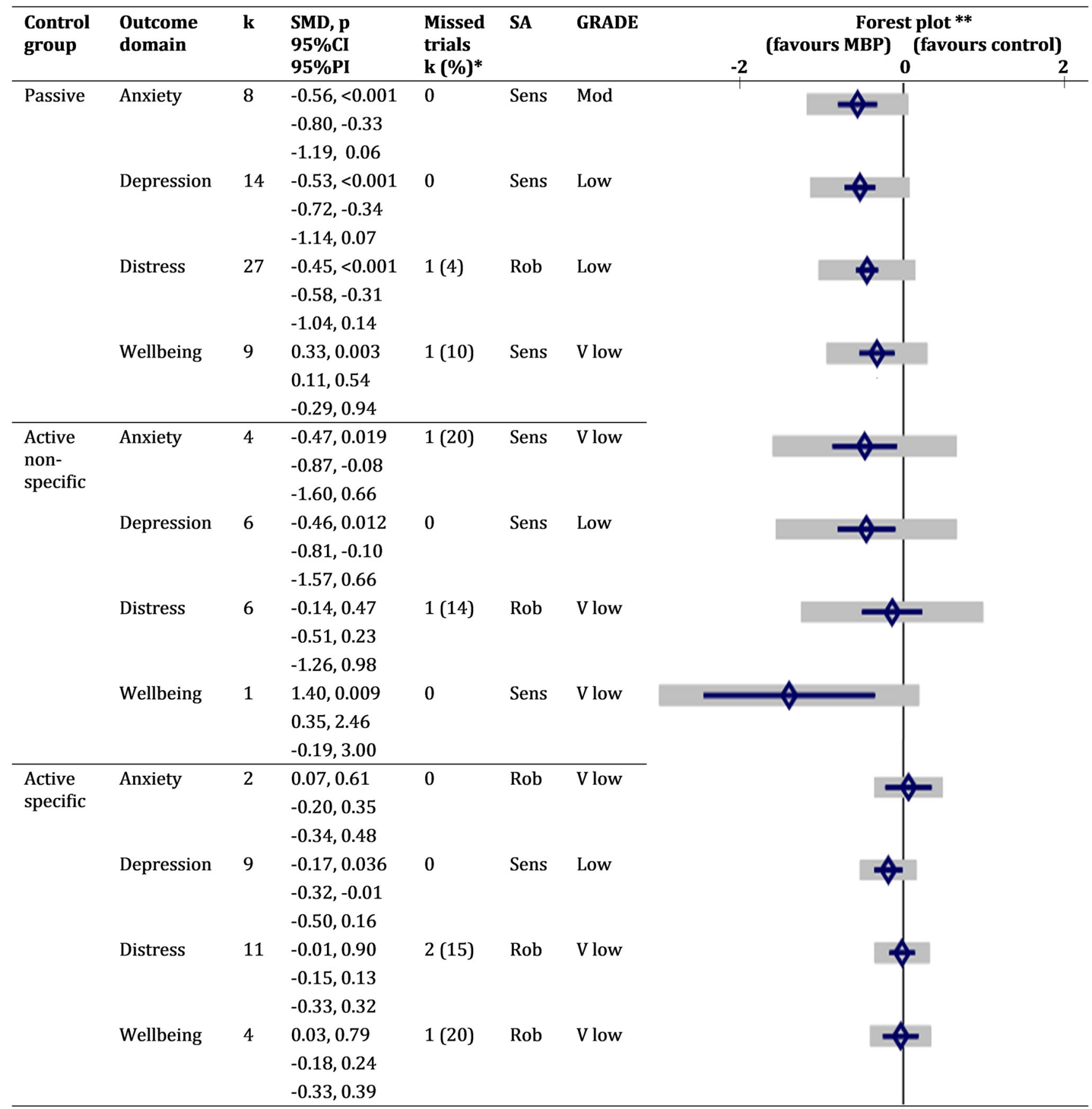

Fig 2. Summary of primary outcome results (outcome time point is 1-6 months post-intervention follow-up). *Number of trials with nonreported data for the corresponding outcome. ${ }^{* *}$ Diamonds are SMDs, blue bars are $95 \%$ CIs, and grey bars are $95 \%$ PIs. CI, confidence interval for overall mean; GRADE, Grading of Recommendations Assessment, Development, and Evaluation approach to assess confidence in the cumulative evidence; $k$, number of trials; MBP, mindfulness-based programme; mod, moderate; PI, prediction interval for new study; Rob, robust; SA, sensitivity analysis; Sens, sensitive; SMD, standardised mean difference; $v$, very.

https://doi.org/10.1371/journal.pmed.1003481.g002

reported that some participants “. . .experienced adverse emotional, mental or bodily states during mindfulness practice. However, this was not considered to be unintended effects of the intervention, but rather expected results of becoming more mindful of inner experiences" (page 5) [124]. Two studies reported a participant abandoning the MBP because s/he felt it was being 
counterproductive $[52,186]$. One study actively monitored clinically meaningful adverse events with no significant differences between trial arms [52]. Four studies set up independent data monitoring and ethics committees $[52,85,92,171]$.

\section{Risk of bias and confidence in the evidence}

Fig 3 summarises the risk-of-bias assessments for individual trials (detailed in S1 Appendix). If a study had different outcome-specific ratings for risk of bias from a given source, the highest-risk rating was used in the summary.

All of the included trials are at high risk of bias according to the RoB2, which considers a trial to be at high risk if it scores high for any 1 source of bias. We noted some concerns about biases arising from the randomisation process for 3 quarters of the studies, mainly due to the lack of mention of allocation sequence concealment efforts; the remaining quarter are mostly low risk. Most of the studies were judged to be at high or moderate risk of bias due to deviations from intended interventions. This was mainly due to lack of measurement or description of contamination between trial arms, which is particularly likely in trials with passive control groups where control participants could have potentially learnt elsewhere critical components of MBPs such as mindfulness skills. However, this bias would dampen rather than inflate any effects favouring MBPs, so it is not of major concern.

About $60 \%$ of the trials were deemed at high risk of bias due to missing outcome data. The direction of this bias could favour the effects of MBPs because participants who feel unwell may be less likely to attend assessment sessions or complete self-reported outcomes [52]. The high prevalence of the latter accounts for why almost all of the included trials are at high risk of bias in measurement of the outcome, because the assessors are the participants themselves. Very few trials have prospective public protocols that include analysis plans, so for most studies, we noted some concerns as we could not rule out biases in the selection of the reported results.

Regarding selective underreporting or nonreporting of results, Fig 2 (also S1 Appendix) show the number of known nonreported results per outcome domain in the included studies. We also found 6 potentially eligible trial registry records with no available results (S1 Appendix); 3 of them may have measured primary outcomes. To estimate unknown nonreported results, we compiled 3 funnel plots. These revealed evidence of small-study or nonreporting biases in the outcome domain of depression for MBPs compared with passive control groups (S1 Appendix), but not for the distress outcome domain, for MBPs compared with passive (S1 Appendix) and active (S1 Appendix) controls.

Given the overall high risk of bias of the included trials, degree of allegiance to the MBP assessed could play an important role, as suggested in previous studies $[283,284]$. If we consider that allegiance may be strong where study authors developed and/or taught the MBP, or where relevant conflicts of interest were disclosed, we could rule out allegiance effects (i.e., discard these factors) in only 7 studies (5\%).

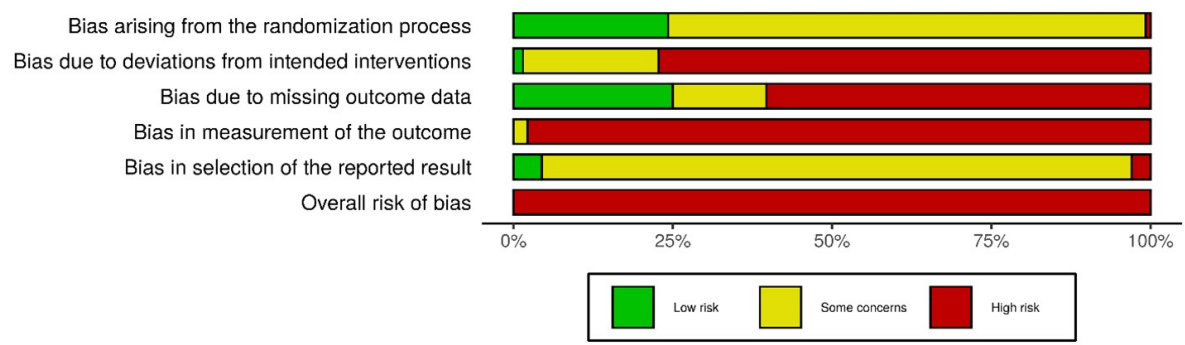

Fig 3. Risk of bias across studies. Highest-risk ratings were used for sources with outcome-level assessments. https://doi.org/10.1371/journal.pmed.1003481.g003 
Fig 2 shows the GRADE assessments for each primary outcome domain (detailed in S1 Appendix). Confidence in the cumulative evidence is low or very low for most outcome domains, except for anxiety in the comparison of MBPs with passive controls, for which we have moderate confidence.

\section{Sensitivity analyses}

To perform a sensitivity analysis of methodological quality, we removed trials deemed to be at high risk of bias from 3 or more sources (most trials have high risk from 2 or 3 sources, so the sample was divided into roughly equal parts). This sensitivity analysis led to reductions in the effects of MBPs on primary outcomes compared to passive controls. The effects on anxiety $(\mathrm{SMD}=-0.22 ; 95 \% \mathrm{CI}-0.57$ to $0.13 ; p-$ value $=0.22)$, depression $(\mathrm{SMD}=-0.24 ; 95 \% \mathrm{CI}-0.49$ to $0.00 ; p$-value $=0.05)$, and mental well-being $(\mathrm{SMD}=0.27 ; 95 \% \mathrm{CI} 0.02$ to $0.58 ; p-$ value $=0.04$ ) were no longer significant, but the effect on psychological distress was robust $(\mathrm{SMD}=-0.30 ; 95 \% \mathrm{CI}-0.48$ to $-0.11 ; p$-value $=0.001)($ S1 Appendix $)$. In the comparison of MBPs with active nonspecific controls, the effect on depression was no longer significant $(\mathrm{SMD}=-0.46 ; 95 \% \mathrm{CI}-0.90$ to $-0.02 ; p$-value $=0.04)$, with no changes in the direction or significance of the other outcomes (S1 Appendix). Further details are presented in S1 Appendix.

In the sensitivity analyses testing within-study correlation assumptions by using Riley's method, for MBPs compared with passive controls the effects on well-being lost significance (S1 Appendix), compared with active nonspecific controls the effects on depression lost significance (S1 Appendix), and compared with active specific controls the effects on depression became significant (S1 Appendix). In the sensitivity analyses testing within-study correlation assumptions by conducting univariate meta-analyses, the effect of MBPs compared with passive controls on anxiety lost significance (S1 Appendix), compared with active nonspecific controls the effect on depression lost significance (S1 Appendix), and there was no change in effects compared with specific controls (S1 Appendix).

Primary outcomes were uniformly robust to the few standard deviation imputations made, and to the ICC imputation. The sensitivity analysis of skewed data could only be conducted in the comparison of MBPs with passive controls. There was no change in the size or significance of estimates, but PIs around the effects of MBPs on anxiety, depression, and distress became narrower, excluding adverse scenarios (S1 Appendix).

Several trials reported only the fact that results for some outcomes did not reach statistical significance, rather than the effects themselves. In these cases, we assumed the effect size to be null (i.e., point estimate $=0$ ) and calculated the variance from the sample size. We then conducted post hoc sensitivity analyses setting the effect size to $+/-1$ standard error. When setting the effect size to +1 standard error, in the comparison of MBPs with active nonspecific controls, the effect on depression lost significance (S1 Appendix); other than that, all of the results were robust to our point estimate imputation (S1 Appendix).

We performed a post hoc sensitivity analysis on our primary outcomes excluding trials with unclear teacher competence. There was no change in the size or significance of the estimates, except for the effect on anxiety in comparison with passive controls, which became stronger and with PIs excluding the null (S1 Appendix).

\section{Moderator analyses}

For the comparison of MBPs with passive controls, the multivariate meta-regression including all time point ranges failed to converge, so we only included the primary range of 1 to 6 months follow-up (S1 Appendix). 
For depression, SMDs for MBPs versus passive controls tested in the USA were 1.10 units lower (i.e., less effective) than those tested elsewhere, adjusting for other potential moderators $(p<0.001)$. In a post hoc analysis to explore whether this difference is explained by lower risk of bias in trials conducted in the USA, we included the number of high risk-of-bias sources as a variable in the model: This did not modify the size or significance of this moderation. Running a meta-analysis of non-USA trials only returned a large effect size (SMD - 0.93 (95\%CI $-1.25,-0.62), p<0.001$ ) and narrower prediction intervals that excluded the null effect $(-1.66,-0.21)$. The corollary analysis including only USA-based trials returned a borderline significant small effect (SMD $-0.24(95 \% \mathrm{CI}-0.48,-0.01) p=0.04)$.

Selective MBPs were 1.10 standard deviations more effective $(p=0.002)$, and indicated MBPs were 0.84 standard deviations more effective $(p=0.014$; fewer than 5 outcomes in this category) than universal MBPs, in the strength of their benefits compared to passive controls for depression. Running separate analyses for selective and indicated versus universal MBPs did not reduce heterogeneity or significantly modify effects, although the benefits of universal MBPs versus passive controls for depression had a smaller effect size (SMD 0.70 for selective and indicated and 0.39 for universal interventions).

Weaker moderator effects for the depression outcome domain showed that for each extra hour of in-person teaching, the beneficial effect was reduced by 0.05 standard deviations $(p=0.013)$ and that MBPs that included physical exercise were 0.96 standard deviations more effective than MBPs with no additional components ( $p=0.017$, fewer than 5 outcomes in base category) relative to passive controls.

For the effects of MBPs versus passive controls on anxiety, MBPs, which were indicated interventions, were 1.12 standard deviations more effective than universal MBPs, adjusting for other potential moderators ( $\mathrm{p}=0.007$, fewer than 5 outcomes in this category). Study location had the same moderating effect as with depression, albeit with borderline significance ( $p=0.028$ ), which disappeared after adjusting for methodological quality. There were no significant moderator effects for the psychological distress and mental well-being outcomes when comparing MBPs with passive controls.

In comparison with active specific controls, MBPs may be less effective to reduce distress as a selective intervention ( $p=0.02$, S1 Appendix). However, this last analysis (univariate since multivariate meta-analyses failed to converge) only had 11 studies and fewer than 5 studies per category, so the results are unreliable. No other outcome domains could be assessed.

\section{Discussion}

\section{Summary of findings}

We report a systematic review and meta-analysis of RCTs comparing the effects of groupbased MBPs delivered in nonclinical settings, versus control conditions, on a range of measures of mental health and functioning.

Our primary outcome results show with a very low to moderate degree of confidence that compared with taking no action (a passive control), MBPs on average improve medium-term mental health outcomes in nonclinical settings. Psychological distress shows the most robust improvement and well-being the smallest improvement, while depression and anxiety show the most homogeneous one.

Compared with taking nonspecific action, MBPs may improve depressive symptoms and the relationship with the self, but reliability is low. Compared with other interventions to improve mental health, we found no indication of MBPs being better or worse.

In general and across comparisons, we cannot be confident that MBPs will confer benefits in every setting. The strongest moderators of MBP effects, which modulated depression and 
anxiety outcomes, were study population-MBPs targeted at higher risk populations or at those with subclinical symptoms of mental disorders were more beneficial-and study nationality, with USA-based trials reporting smaller effects than elsewhere. All of the trials included in the review were deemed at high risk of bias, and of all the primary outcomes, only the effects of MBPs relative to passive controls on distress remained when trials with the highest risk of bias were excluded in sensitivity analyses.

\section{Interpretation and comparison with previous research}

Our results present a more complex picture than those of previous reviews, particularly concerning the heterogeneity of effects revealed by wide PIs. PIs show the range of effects to be expected in similar studies to those included in the meta-analysis. In the absence of betweenstudy heterogeneity, PIs equate to CIs (which summarise average effects for the average study); in the presence of such heterogeneity, PIs are wider, meaning that there will be settings where conclusions based on CIs will not hold. Settings encompass a broad range of factors such as type of community, social context, type of MBP, and the way in which the study was conducted; any of these could moderate intervention effects.

Other reviews have also found that their results were sensitive to trial quality [32]. There have been several calls to improve the quality of mindfulness research, and of behavioural interventions research generally, with only modest improvement over time [39,285-287].

Our finding that selective and indicated MBPs were more effective in reducing anxiety and depression than universal MBPs is not unique [29]. It may reflect the fact that those with worse mental health to begin with are more likely to benefit. This finding could also be due to differences in the types of MBPs or their teachers (e.g., those teaching selective or indicated MBPs being therapists). However, the absence of this differential effect on psychological distress outcomes suggests that results could be explained by a ceiling effect: Depression and anxiety questionnaires may be more sensitive to improvement among high-risk or subclinical populations than among those who are less affected, while distress questionnaires may retain sensitivity along this mental health spectrum.

In synthesising studies from different countries and cultures, we tested whether the intervention could have an effect that goes beyond cultural differences. The results obtained, in particular the wide prediction intervals plus the moderation by study location, suggest that cultural and social differences do determine the extent to which MBPs are beneficial [40]. Our moderation analysis tapped into one such difference, as have other recent analyses [49]. Modern mindfulness is an American product undergoing continuous dissemination within the USA since the 1970s [9], so familiarity with it is high. In contrast, a novelty effect, fuelled by advocates in a number of ways (e.g., through researchers' intervention allegiance), may be operating outside of the USA to varying degrees [49]. Also, MBPs may be taught in subtly different ways depending on the culture in which they are modified and delivered.

Little is known about differential effects of various MBP intervention components [288]. In our moderation analyses, we found some support for incorporating physical activity within MBPs. Other effect moderators need to be considered. A recent systematic review of workplace MBPs noted that some individual study effect estimates are opposite to the direction of benefit (see our examples in S1 Appendix), and suggested that not allowing the MBP to take place within working hours could be the cause, since needing extra time to attend the MBP on top of work demands may increase stress [24].

Our weak finding that longer courses may be slightly less beneficial was unexpected, although it could be a result of multiple testing and residual confounding. Other reviews 
assessing course duration have not found this to be an important effect moderator $[31,32]$. Combined, this evidence suggests that MBP courses do not have to be long to be effective.

There is consensus among mindfulness leaders that good teacher training is critical for MBP success [6,289]. However, our preliminary post hoc analysis, in line with previous research, did not find evidence of this factor being influential [290]. Rather than mindfulness credentialing, other related aspects such as type of mindfulness training, teaching and communication skills, or whether the teacher had a similar background to their students, may influence MBP effects.

Whether beneficial effects wear off with longer follow-up periods may depend on continued mindfulness practice, which evidence suggests tails off with time [291]. It may also indicate that a proportion of the effect is nonspecific, including social interaction (by virtue of the group format) and placebo effects, particularly for self-reported outcomes among unblinded participants.

Our results differ in some respects from those of recent reviews looking at MBPs for university students $[29,31]$ and from those looking at MBPs for patients with mental health problems $[57,292]$. It may be that MBPs are more beneficial to younger populations and to those feeling worse. However, recent reviews of MBPs for children and adolescents have mixed findings [49,56,293]. Contextual factors (e.g., student mindset), intervention characteristics, and review methodologies also need to be considered when comparing results between reviews.

No adverse effects were reported. However, confidence in this result remains low given the low percentage of trials measuring them, as noted before [31,294], and the passive reliance on spontaneous reporting in most studies, which may underestimate adverse effect frequency by more than 20-fold $[38,295,296]$. The wide prediction intervals found in this review may go some way to explain why unwanted effects are reported in surveys, despite MBPs showing benefit on average $[297,298]$. It was suggested that unpleasant experiences are part of the intervention effect [124]; it would be important to better understand how common, intense, and heterogeneous these experiences are, both for better intervention targeting and so that commissioners, teachers, and participants know what to expect.

\section{Strengths and limitations of this review}

The strengths of this review include a comprehensive search, detailed prespecification of methods, robust analytic techniques, the fact that none of us have developed or taught any of the included MBPs, and the synthesis of a substantial amount of evidence; these strengths overcome most of the limitations highlighted in an extensive critique of existing healthcare reviews [299]. However, the low quality of most of the primary studies significantly affects confidence and therefore utility of the review results.

MBPs are complex interventions, so quantitative synthesis involved researchers' judgement and simplification [300]. Many different interventions exist, which include the word mindfulness in their title; we carefully selected those that seemed to follow consensus MBP guidelines to obtain meaningful and focused results, but this was not always clear. Some criteria, like including MBPs with a minimum of 4 hours of instruction, or defining the main outcome time point range as between 1 and 6 months post-intervention, were reasoned and predefined, but ultimately arbitrary limits. We made some grouping decisions with undesirably thin data, for example, for control groups and intervention components. The characteristics of the participants in MBPs classed as indicated interventions may overlap with those of participants in clinical settings, although we excluded MBPs which required participants to have a clinical diagnosis. We have not analysed individual-level moderators of effect such as baseline mental health. To address this, we plan to conduct an individual participant data meta-analysis. 


\section{Implications for practice and research}

Compared with taking no action, MBPs can be an effective means to promote mental health. But it cannot be expected that MBPs will work in every nonclinical setting. This review showed that MBPs implemented within a wide range of cultures and settings, by different agents, and targeting various groups in the community, can have different effects. The techniques and frameworks taught in MBPs have in turn rich and diverse backgrounds (e.g., early Buddhist psychology, contemplative traditions, cognitive neuroscience, participatory medicine) [6]. The interplays between all these social factors can be expected to exert their own effects over and above any universally human psychophysiological effects.

To understand what happens in which setting, implementation of MBPs should be preceded by or partnered with further studies. This research should be interdisciplinary, involving social scientists to better understand the interplay between complex healthcare interventions, like MBPs, and cultural landscapes [301]. Involving stakeholders in participatory research processes is also likely to shed more light on for whom MBPs may be helpful and in what ways. They could also help intervention developers to adapt MBPs to specific populations considering factors other than teachers' mindfulness training or intervention duration.

In the meantime, it is important for mindfulness practitioners not to assume that MBPs will work universally and to discuss this with their students. It has been shown that MBPs need to be implemented carefully within clinical settings [289]; care is also advised in nonclinical settings, where participants may be more diverse and less supported. In planning MBP provision, those adapted to specific at-risk populations may be a better option than universal MBPs.

The field of online MBPs is growing rapidly both in terms of offer and demand, and the Coronavirus Disease 2019 (COVID-19) pandemic has only accelerated this growth [302]. Meta-analyses suggest that online MBPs may be as effective as their offline counterparts, despite most lacking interactions with teachers and peers [31,47]. If the effects of MBPs vary as widely according to the setting as their offline counterparts, the automatic nature of many online MBPs and their expanded audience raise concerns about the lack of human support. More research comparing effectiveness and safety profiles of different MBP delivery formats head-to-head is needed.

This review suggests that MBPs may have specific effects on common mental health symptoms. However, other preventative interventions may be similarly effective. Apart from effectiveness, other aspects such as cultural acceptability, feasibility, and costs need to be considered when deciding which preventative intervention to implement. Comparative effectiveness research is needed to understand which interventions work best in which setting.

The modest trial quality improvement over time may in part reflect low investment in mental health research [303], and challenges around implementing participant blinding and avoiding outcome self-reporting inherent to behavioural mental health intervention trials. However, it is possible to reduce bias with low-resource measures. Allocation sequence concealment can be done simply, and needs to be reported in publications. Authors could easily encourage participants to complete outcome surveys even when they abandon the MBP and use these data in intention-to-treat analyses. They could also actively ask participants about any unexpected or unwanted effects. It is crucial for future trialists to prospectively register trial protocols in free public registers where they specify a primary outcome measure and time point and include a primary outcome data analysis plan. In their publications, authors need to add more intervention and teacher details, even if it has to be in supplementary materials. More resource-intensive improvements include establishing research teams with no allegiance to the intervention, using active control groups (particularly active nonspecific control groups), and collecting data beyond self-report. Regarding methodological implications for 
future reviews, our primary outcome results were sensitive to analytic choices, demonstrating how important it is to publicly prespecify meta-analyses in detail to avoid outcome-led analytic strategy selection.

In sum, compared with taking no action, MBPs promote mental health in the average nonclinical setting but cannot be expected to work in every setting. Although MBPs may have specific effects on some common mental health symptoms, other interventions may be equally effective. MBPs should be implemented with care in nonclinical settings and partnered with well-conducted research.

\section{Supporting information}

\section{S1 Appendix. Supporting information file.}

(PDF)

\section{S1 Checklist. PRISMA checklist.}

(PDF)

\section{Acknowledgments}

We are extremely grateful to our professional and public stakeholder groups for their keen involvement. We also thank Joseph Durlak, Alp Arat, Steven Stanley, and Isla Kuhn for their help.

\section{Author Contributions}

Conceptualization: Julieta Galante, Tim Dalgleish, Ian R White, Peter B Jones.

Data curation: Julieta Galante, Claire Friedrich, Anna F Dawson, Marta Modrego-Alarcón, Pia Gebbing, Tim Dalgleish, Peter B Jones.

Formal analysis: Julieta Galante, Claire Friedrich, Anna F Dawson, Marta Modrego-Alarcón, Pia Gebbing, Irene Delgado-Suárez, Radhika Gupta, Lydia Dean, Ian R White.

Funding acquisition: Julieta Galante.

Investigation: Julieta Galante, Claire Friedrich, Anna F Dawson.

Methodology: Julieta Galante, Tim Dalgleish, Ian R White, Peter B Jones.

Project administration: Julieta Galante, Claire Friedrich, Anna F Dawson.

Supervision: Julieta Galante, Tim Dalgleish, Ian R White, Peter B Jones.

Visualization: Julieta Galante, Ian R White.

Writing - original draft: Julieta Galante, Claire Friedrich.

Writing - review \& editing: Julieta Galante, Claire Friedrich, Anna F Dawson, Marta Modrego-Alarcón, Pia Gebbing, Irene Delgado-Suárez, Radhika Gupta, Lydia Dean, Tim Dalgleish, Ian R White, Peter B Jones.

\section{References}

1. Vos T, Barber RM, Bell B, Bertozzi-Villa A, Biryukov S, Bolliger I, et al. Global, regional, and national incidence, prevalence, and years lived with disability for 301 acute and chronic diseases and injuries in 188 countries, 1990-2013: a systematic analysis for the Global Burden of Disease Study 2013. Lancet. 2015; 386 (9995):743-800. https://doi.org/10.1016/S0140-6736(15)60692-4 PMID: 26063472 
2. Mental Health Foundation. Mental health foundation strategy 2020-2025: Making prevention happen. London, UK: Mental Health Foundation; 2019.

3. Samele $\mathrm{C}$. Increasing momentum in prevention of mental illness and mental health promotion across Europe. BJPsych International. 2016; 13 (1):22-3. https://doi.org/10.1192/s2056474000000957 PMID: 29093889

4. Mental Health Foundation. Nearly one in three people are regularly stressed, survey for Mental Health Awareness Week reveals. 2015 [10/11/2016]. http://www.mentalhealth.org.uk/news/nearly-onethree-people-are-regularly-stressed-survey-mental-health-awareness-week-reveals.

5. Kabat-Zinn J. Full Catastrophe Living, Revised Edition: How to cope with stress, pain and illness using mindfulness meditation. 2 ed. London: Piatkus; 2013.

6. Crane RS, Brewer J, Feldman C, Kabat-Zinn J, Santorelli S, Williams JM, et al. What defines mindfulness-based programs? The warp and the weft. Psychol Med 2017; 47(6):990-9. Epub 2016/12/30. https://doi.org/10.1017/S0033291716003317 PMID: 28031068.

7. National Institute for Health and Care Excellence (NICE). Guideline CG91. Depression in adults: recognition and management. 2009.

8. Kabat-Zinn J. Foreword: Seeds of a necessary global renaissance in the making: the refining of psychology's understanding of the nature of mind, self, and embodiment through the lens of mindfulness and its origins at a key inflection point for the species. Curr Opin Psychol. 2019; 28:xi-xvii. Epub 2019/ 05/18. https://doi.org/10.1016/j.copsyc.2019.02.005 PMID: 31097398.

9. Kucinskas J. The Mindful Elite: Mobilizing from the Inside Out. New York: Oxford University Press; 2018. 260 p.

10. Burke A, Lam CN, Stussman B, Yang H. Prevalence and patterns of use of mantra, mindfulness and spiritual meditation among adults in the United States. BMC Complement Altern Med. 2017; 17 (1):316. Epub 2017/06/18. https://doi.org/10.1186/s12906-017-1827-8 PMID: 28619092.

11. Klingbeil DA, Renshaw TL. Mindfulness-based interventions for teachers: A meta-analysis of the emerging evidence base. School psychology quarterly: the official journal of the Division of School Psychology, American Psychological Association. 2018; 33(4):501-11. Epub 2018/12/07. https://doi. org/10.1037/spq0000291 PMID: 30507235.

12. Lomas T, Medina JC, Ivtzan I, Rupprecht S, Eiroa-Orosa FJ. The impact of mindfulness on the wellbeing and performance of educators: A systematic review of the empirical literature. Teach Teach Educ. 2017; 61:132-41. https://doi.org/10.1016/j.tate.2016.10.008

13. Burgdorf $V$, Szabo M, Abbott MJ. The Effect of Mindfulness Interventions for Parents on Parenting Stress and Youth Psychological Outcomes: A Systematic Review and Meta-Analysis. Front Psychol. 2019; 10:1336. Epub 2019/06/28. https://doi.org/10.3389/fpsyg.2019.01336 PMID: 31244732.

14. Collins RN, Kishita N. The Effectiveness of Mindfulness-and Acceptance-Based Interventions for Informal Caregivers of People With Dementia: A Meta-Analysis. Gerontologist. 2019; 59(4):e363e79. Epub 2018/04/11. https://doi.org/10.1093/geront/gny024 PMID: 29635303.

15. Li G, Yuan H, Zhang W. The Effects of Mindfulness-Based Stress Reduction for Family Caregivers: Systematic Review. Arch Psychiatr Nurs. 2016; 30 (2):292-9. https://doi.org/10.1016/j.apnu.2015.08. 014 PMID: 26992885

16. Spinelli C, Wisener M, Khoury B. Mindfulness training for healthcare professionals and trainees: $A$ meta-analysis of randomized controlled trials. J Psychosom Res. 2019; 120:29-38. Epub 2019/04/ 02. https://doi.org/10.1016/j.jpsychores.2019.03.003 PMID: 30929705.

17. Klein A, Taieb $O$, Xavier S, Baubet $T$, Reyre A. The benefits of mindfulness-based interventions on burnout among health professionals: A systematic review. Explore (NY). 2020; 16(1):35-43. Epub 2019/11/16. https://doi.org/10.1016/j.explore.2019.09.002 PMID: 31727578.

18. Scheepers RA, Emke H, Epstein RM, Lombarts K. The impact of mindfulness-based interventions on doctors' well-being and performance: A systematic review. Med Educ. 2020; 54(2):138-49. Epub 2019/12/24. https://doi.org/10.1111/medu.14020 PMID: 31868262.

19. Ghawadra SF, Abdullah KL, Choo WY, Phang CK. Mindfulness-based stress reduction for psychological distress among nurses: A systematic review. J Clin Nurs. 2019; 28(21-22):3747-58. Epub 2019/ 07/04. https://doi.org/10.1111/jocn.14987 PMID: 31267619.

20. Suleiman-Martos N, Gomez-Urquiza JL, Aguayo-Estremera R, Canadas-De La Fuente GA, De La Fuente-Solana El, Albendin-Garcia L. The effect of mindfulness training on burnout syndrome in nursing: A systematic review and meta-analysis. J Adv Nurs. 2020; 76(5):1124-40. Epub 2020/02/07. https://doi.org/10.1111/jan.14318 PMID: 32026484.

21. Burton A, Burgess C, Dean S, Koutsopoulou GZ, Hugh-Jones S. How Effective are Mindfulness-Based Interventions for Reducing Stress Among Healthcare Professionals? A Systematic Review and Meta- 
Analysis. Stress and Health [Internet]. 2016. Available from: https://doi.org/10.1002/smi.2673 PMID: 26916333

22. Sappington R, Longshore K. Systematically reviewing the efficacy of mindfulness-based interventions for enhanced athletic performance. J Clin Sport Psychol. 2015; 9(3):232-62. https://doi.org/10.1123/ jcsp.2014-0017 2015-45507-003.

23. Janssen M, Heerkens Y, Kuijer W, van der Heijden B, Engels J. Effects of Mindfulness-Based Stress Reduction on employees' mental health: A systematic review. PLoS ONE. 2018; 13(1):e0191332. Epub 2018/01/25. https://doi.org/10.1371/journal.pone.0191332 PMID: 29364935.

24. Bartlett L, Martin A, Neil AL, Memish K, Otahal P, Kilpatrick M, et al. A systematic review and metaanalysis of workplace mindfulness training randomized controlled trials. J Occup Health Psychol. 2019; 24(1):108-26. Epub 2019/02/05. https://doi.org/10.1037/ocp0000146 PMID: 30714811.

25. Vonderlin R, Biermann M, Bohus M, Lyssenko L. Mindfulness-Based Programs in the Workplace: a Meta-Analysis of Randomized Controlled Trials. Mind. 2020; 11:1579-98. https://doi.org/10.1007/ s12671-020-01328-3

26. Lomas T, Medina JC, Ivtzan I, Rupprecht S, Eiroa-Orosa FJ. Mindfulness-based interventions in the workplace: An inclusive systematic review and meta-analysis of their impact upon wellbeing. J Posit Psychol. 2018; 14 (5):625-40. https://doi.org/10.1080/17439760.2018.1519588

27. Virgili M. Mindfulness-Based Interventions Reduce Psychological Distress in Working Adults: a Meta-Analysis of Intervention Studies. Mind. 2013; 6 (2):326-37. https://doi.org/10.1007/s12671-0130264-0

28. Li SYH, Bressington $D$. The effects of mindfulness-based stress reduction on depression, anxiety, and stress in older adults: A systematic review and meta-analysis. Int J Ment Health Nurs. 2019; 28 (3):635-56. Epub 2019/01/19. https://doi.org/10.1111/inm.12568 PMID: 30656813.

29. Ma L, Zhang Y, Cui Z. Mindfulness-Based Interventions for Prevention of Depressive Symptoms in University Students: a Meta-analytic Review. Mind. 2019; 10 (11):2209-24. https://doi.org/10.1007/ s12671-019-01192-w

30. Halladay JE, Dawdy JL, McNamara IF, Chen AJ, Vitoroulis I, McInnes N, et al. Mindfulness for the Mental Health and Well-Being of Post-Secondary Students: A Systematic Review and Meta-Analysis. Mind. 2018; 10 (3):397-414.

31. Dawson AF, Brown WW, Anderson J, Datta B, Donald JN, Hong K, et al. Mindfulness-based Interventions for University Students: A Systematic Review and Meta-analysis of Randomized Controlled Trials. Applied Psychology: Health and Well-Being. 2019; Epub ahead of print.

32. Michael de Vibe AB, Fattah Sabina, Dyrdal Gunvor M, Even, Halland EET-S. Mindfulness-based stress reduction (MBSR) for improving health, quality of life and social functioning in adults: a systematic review and meta-analysis. Campbell Collaboration. 2017. https://doi.org/10.4073/csr.2017.11

33. Victorson D, Kentor M, Maletich C, Lawton RC, Kaufman VH, Borrero M, et al. Mindfulness Meditation to Promote Wellness and Manage Chronic Disease: A Systematic Review and Meta-Analysis of Mindfulness-Based Randomized Controlled Trials Relevant to Lifestyle Medicine. Am J Lifestyle Med. 2015; 9 (3):185-211. https://doi.org/10.1177/1559827614537789

34. Sharma M, Rush SE. Mindfulness-based stress reduction as a stress management intervention for healthy individuals: a systematic review. Journal of evidence-based complementary \& alternative medicine. 2014; 19(4):271-86. Epub 2014/07/24. https://doi.org/10.1177/2156587214543143 PMID: 25053754.

35. De Vibe M, Bjørndal A, Tipton E, Hammerstrøm K, Kowalski K. Mindfulness Based Stress Reduction (MBSR) for Improving Health, Quality of Life, and Social Functioning in Adults. Campbell Syst Rev. 2012; 8 (1):1-127. https://doi.org/10.4073/csr.2012.3

36. Chiesa A, Serretti A. Mindfulness-Based Stress Reduction for Stress Management in Healthy People: A Review and Meta-Analysis. J Altern Complement Med. 2009; 15 (5):593-600. https://doi.org/10. 1089/acm.2008.0495 PMID: 19432513

37. Lomas T, Medina JC, Ivtzan I, Rupprecht S, Eiroa-Orosa FJ. A Systematic Review and Meta-analysis of the Impact of Mindfulness-Based Interventions on the Well-Being of Healthcare Professionals. Mind. 2018; 10 (7):1193-216. https://doi.org/10.1007/s12671-018-1062-5

38. Van Dam NT, van Vugt MK, Vago DR, SchmalzI L, Saron CD, Olendzki A, et al. Mind the Hype: A Critical Evaluation and Prescriptive Agenda for Research on Mindfulness and Meditation. Perspectives on psychological science: a journal of the Association for Psychological Science. 2017:1745691617709589. Epub 2017/10/11. https://doi.org/10.1177/1745691617709589 PMID: 29016274.

39. Goldberg SB, Tucker RP, Greene PA, Simpson TL, Kearney DJ, Davidson RJ. Is mindfulness research methodology improving over time? A systematic review. PLoS ONE. 2017; 12(10): e0187298. Epub 2017/11/01. https://doi.org/10.1371/journal.pone.0187298 PMID: 29088283. 
40. Kelly MP, Noyes J, Kane RL, Chang C, Uhl S, Robinson KA, et al. AHRQ series on complex intervention systematic reviews-paper 2: defining complexity, formulating scope, and questions. J Clin Epidemiol. 2017; 90:11-8. Epub 2017/07/20. https://doi.org/10.1016/j.jclinepi.2017.06.012 PMID: 28720514.

41. Tanner-Smith EE, Grant S. Meta-Analysis of Complex Interventions. Annu Rev Public Health. 2018; 39 (1):135-51. https://doi.org/10.1146/annurev-publhealth-040617-014112 PMID: 29328876

42. Galante J, Dawson A, Modrego M, Dalgleish T, White I, Jones PB. PROSPERO CRD42018105213 Mindfulness-based programmes for mental health promotion: a systematic review and meta-analysis of randomised controlled trials 2018. Available from: https://www.crd.york.ac.uk/prospero/display_ record. php?ID=CRD42018105213.

43. Moher D, Liberati A, Tetzlaff J, Altman DG. Preferred reporting items for systematic reviews and meta-analyses: the PRISMA statement. BMJ (Clinical research ed). 2009; 339:b2535. https://doi.org/ 10.1136/bmj.b2535 PMID: 19622551

44. Mayo-Wilson E, Li T, Fusco N, Bertizzolo L, Canner JK, Cowley T, et al. Cherry-picking by trialists and meta-analysts can drive conclusions about intervention efficacy. J Clin Epidemiol. 2017; 91:95-110. Epub 2017/08/27. https://doi.org/10.1016/j.jclinepi.2017.07.014 PMID: 28842290.

45. Gu J, Strauss $\mathrm{C}$, Bond R, Cavanagh $\mathrm{K}$. How do mindfulness-based cognitive therapy and mindfulness-based stress reduction improve mental health and wellbeing? A systematic review and metaanalysis of mediation studies. Clin Psychol Rev. 2015; 37C:1-12. Epub 2015/02/18. https://doi.org/10. 1016/j.cpr.2015.01.006 PMID: 25689576.

46. Klatt MD, Buckworth J, Malarkey WB. Effects of low-dose mindfulness-based stress reduction (MBSR-Id) on working adults. Health Educ Behav. 2009; 36(3):601-14. http://dx.doi.org/10.1177/ 1090198108317627. PMID: 18469160.

47. Jayawardene WP, Lohrmann DK, Erbe RG, Torabi MR. Effects of preventive online mindfulness interventions on stress and mindfulness: A meta-analysis of randomized controlled trials. Prev Med Rep. 2017; 5:150-9. https://doi.org/10.1016/j.pmedr.2016.11.013 PMID: 28050336

48. Covidence systematic review software. Melbourne, Australia: Veritas Health Innovation; 2019.

49. Odgers K, Dargue N, Creswell C, Jones MP, Hudson JL. The Limited Effect of Mindfulness-Based Interventions on Anxiety in Children and Adolescents: A Meta-Analysis. Clin Child Fam Psychol Rev. 2020. Epub 2020/06/26. https://doi.org/10.1007/s10567-020-00319-z PMID: 32583200.

50. White IR, Thomas J. Standardized mean differences in individually-randomized and cluster-randomized trials, with applications to meta-analysis. Clin Trials. 2005; 2(2):141-51. Epub 2005/11/11. https://doi.org/10.1191/1740774505cn081oa PMID: 16279136.

51. McKenzie JE, Herbison GP, Deeks JJ. Impact of analysing continuous outcomes using final values, change scores and analysis of covariance on the performance of meta-analytic methods: a simulation study. Res Synth Methods. 2016; 7(4):371-86. Epub 2015/12/31. https://doi.org/10.1002/jrsm.1196 PMID: 26715122.

52. Galante J, Dufour G, Vainre M, Wagner AP, Stochl J, Benton A, et al. A mindfulness-based intervention to increase resilience to stress in university students (the Mindful Student Study): a pragmatic randomised controlled trial. Lancet Public Health. 2018; 3 (2):e72-81. https://doi.org/10.1016/S24682667(17)30231-1 PMID: 29422189

53. Higgins JPT, Thomas J, Chandler J, Cumpston M, Li T, Page MJ, et al., editors. Cochrane Handbook for Systematic Reviews of Interventions version 6.0 (updated July 2019). www.training.cochrane.org/ handbook: Cochrane; 2019.

54. Harrer M, Cuijpers P, Furukawa TA, Ebert DD. Doing Meta-Analysis in R: A Hands-on Guide 2019. https://bookdown.org/MathiasHarrer/Doing_Meta_Analysis_in_R/.

55. Kontopantelis E, Reeves D. METAEFF: Stata module to perform effect sizes calculations for metaanalyses. Statistical Software Components S457072. revised 18 Mar 2015 ed: Boston College Department of Economics; 2015.

56. Dunning DL, Griffiths K, Kuyken W, Crane C, Foulkes L, Parker J, et al. The Effects of MindfulnessBased Interventions on Cognition and Mental Health in Children and Adolescents: A Meta-Analysis of Randomised Controlled Trials. J Child Psychol Psychiatry. 2019; 60 (3):244-58. https://doi.org/10. 1111/jcpp.12980 PMID: 30345511

57. Goyal M, Singh S, Sibinga EMS, Gould NF, Rowland-Seymour A, Sharma R, et al. Meditation programs for psychological stress and well-being: a systematic review and meta-analysis. JAMA Intern Med. 2014; 174(3):357-68. https://doi.org/10.1001/jamainternmed.2013.13018 PMID: 24395196.

58. Sterne JAC, Savović J, Page MJ, Elbers RG, Blencowe NS, Boutron I, et al. RoB 2: a revised tool for assessing risk of bias in randomised trials. BMJ (Clinical research ed). 2019; 366:14898. https://doi. org/10.1136/bmj.I4898 PMID: 31462531 
59. Coronado-Montoya S, Levis AW, Kwakkenbos L, Steele RJ, Turner EH, Thombs BD. Reporting of Positive Results in Randomized Controlled Trials of Mindfulness-Based Mental Health Interventions. PLoS ONE. 2016; 11(4):e0153220. Epub 2016/04/09. https://doi.org/10.1371/journal.pone.0153220 PMID: 27058355.

60. Guyatt G, Oxman A, Vist G, Kunz R, Falck-Ytter Y, Alonso-Coello P, et al. GRADE: an emerging consensus on rating quality of evidence and strength of recommendations. BMJ Open. 2008; 336 (7650):924-6. https://doi.org/10.1136/bmj.39489.470347.AD PMID: 18436948

61. StataCorp. Stata Statistical Software: Release 16. College Station, TX: StataCorp LLC; 2019.

62. Vickerstaff V, Ambler G, King M, Nazareth I, Omar RZ. Are multiple primary outcomes analysed appropriately in randomised controlled trials? A review. Contemp Clin Trials. 2015; 45(Pt A):8-12. Epub 2015/07/29. https://doi.org/10.1016/j.cct.2015.07.016 PMID: 26215934.

63. Mavridis D, Salanti G. A practical introduction to multivariate meta-analysis. Stat Methods Med Res. 2013; 22(2):133-58. Epub 2012/01/26. https://doi.org/10.1177/0962280211432219 PMID: 22275379.

64. White IR. Multivariate random-effects meta-analysis. Stata J. 2009.

65. White IR. Multivariate random-effects meta-regression: Updates to mvmeta. Stata J. 2011. PMID: 22065944

66. Riley RD, Thompson JR, Abrams KR. An alternative model for bivariate random-effects meta-analysis when the within-study correlations are unknown. Biostatistics. 2007; 9 (1):172-86. https://doi.org/ 10.1093/biostatistics/kxm023 PMID: 17626226

67. Price MJ, Blake HA, Kenyon S, White IR, Jackson D, Kirkham JJ, et al. Empirical comparison of univariate and multivariate meta-analyses in Cochrane Pregnancy and Childbirth reviews with multiple binary outcomes. Res Synth Methods. 2019; 10 (3):440-51. https://doi.org/10.1002/jrsm.1353 PMID: 31058440

68. Riley RD, Higgins JPT, Deeks JJ. Interpretation of random effects meta-analyses. BMJ (Clinical research ed). 2011; 342:d549. https://doi.org/10.1136/bmj.d549 PMID: 21310794

69. IntHout J, loannidis JP, Rovers MM, Goeman JJ. Plea for routinely presenting prediction intervals in meta-analysis. BMJ Open. 2016; 6(7):e010247. Epub 2016/07/14. https://doi.org/10.1136/bmjopen2015-010247 PMID: 27406637.

70. Gordon RS Jr. An operational classification of disease prevention. Public Health Rep. 1983; 98 (2):107-9. Epub 1983/03/01. PMID: 6856733.

71. Fua R, Gartlehner G, Grant M, Shamliyan T, Sedrakyan A, Wilt TJ, et al. Conducting Quantitative Synthesis When Comparing Medical Interventions: AHRQ and the Effective Health Care Program. Rockville, MD: Agency for Healthcare Research and Quality; 20104 September 2018.

72. de Vibe M, Solhaug I, Rosenvinge JH, Tyssen R, Hanley A, Garland E. Six-year positive effects of a mindfulness-based intervention on mindfulness, coping and well-being in medical and psychology students; Results from a randomized controlled trial. PLoS ONE. 2018; 13(4):e0196053. Epub 2018/ 04/25. https://doi.org/10.1371/journal.pone.0196053 PMID: 29689081.

73. Aeamla-Or N. The effect of mindfulness-based stress reduction on stress, depression, self-esteem and mindfulness in Thai nursing students: a randomised controlled trial: University of Newcastle; 2015.

74. Agee JD, Danoff-Burg S, Grant CA. Comparing Brief Stress Management Courses in a Community Sample: Mindfulness Skills and Progressive Muscle Relaxation. Explore: The Journal of Science and Healing. 2009; 5(2):104-9. http://dx.doi.org/10.1016/j.explore.2008.12.004. PMID: 354247150.

75. Allen M, Dietz M, Blair KS, van Beek M, Rees G, Vestergaard-Poulsen $P$, et al. Cognitive-affective neural plasticity following active-controlled mindfulness intervention. J Neurosci. 2012; 32 (44):15601-10. https://dx.doi.org/10.1523/JNEUROSCI.2957-12.2012. PMID: 23115195.

76. Amutio A, Martínez-Taboada C, Delgado LC, Hermosilla D, Mozaz MJ. Acceptability and Effectiveness of a Long-Term Educational Intervention to Reduce Physicians' Stress-Related Conditions. J Contin Educ Health Prof. 2015; 35(4):255-60. https://doi.org/10.1097/CEH.0000000000000002 PMID: 26953856. Language: English. Entry Date: 20180213. Revision Date: 20180213. Publication Type: Article.

77. Amutio A, Martínez-Taboada C, Hermosilla D, Delgado LC. Enhancing relaxation states and positive emotions in physicians through a mindfulness training program: A one-year study. Psychol Health Med. 2015; 20(6):720-31. https://doi.org/10.1080/13548506.2014.986143 PMID: 25485658. Language: English. Entry Date: 20150709. Revision Date: 20170222. Publication Type: Journal Article.

78. Anclair M, Lappalainen R, Muotka J, Hiltunen AJ. Cognitive behavioural therapy and mindfulness for stress and burnout: a waiting list controlled pilot study comparing treatments for parents of children with chronic conditions. Scand J Caring Sci. 2018; 32(1):389-96. https://doi.org/10.1111/scs.12473 
PMID: 28851063. Language: English. Entry Date: 20180308. Revision Date: 20180308. Publication Type: Article.

79. Anderson ND, Lau MA, Segal ZV, Bishop SR. Mindfulness-based stress reduction and attentional control. Clin Psychol Psychother. 2007; 14(6):449-63. https://doi.org/10.1002/cpp.544 2008-00234-004.

80. Armstrong L, Rimes KA. Mindfulness-Based Cognitive Therapy for Neuroticism (Stress Vulnerability): A Pilot Randomized Study. Behav Ther. 2016; 47(3):287-98. https://doi.org/10.1016/j.beth.2015.12. 005 PMID: 27157024. Language: English. Entry Date: 20180723. Revision Date: 20170928 Publication Type: journal article.

81. Arredondo $\mathrm{M}$, Sabaté $\mathrm{M}$, Valveny $\mathrm{N}$, Langa $\mathrm{M}$, Dosantos $\mathrm{R}$, Moreno J, et al. A mindfulness training program based on brief practices (M-PBI) to reduce stress in the workplace: a randomised controlled pilot study. Int J Occup Environ Health. 2017; 23 (1):40-51. https://doi.org/10.1080/10773525.2017. 1386607 PMID: 29082831

82. Astin JA. Stress reduction through mindfulness meditation. Effects on psychological symptomatology, sense of control, and spiritual experiences. Psychother Psychosom. 1997; 66(2):97-106. https://doi. org/10.1159/000289116 PMID: 9097338.

83. Asuero AM, Queraltó JM, Pujol-Ribera E, Berenguera A, Rodriguez-Blanco T, Epstein RM. Effectiveness of a Mindfulness Education Program in Primary Health Care Professionals: A Pragmatic Controlled Trial. J Contin Educ Health Prof. 2014; 34(1):4-12. https://doi.org/10.1002/chp.21211 PMID: 24648359. Language: English. Entry Date: 20140324. Revision Date: 20150710. Publication Type: Journal Article.

84. Auseron GA, Viscarret MRE, Goni CF, Rubio VG, Pascual PP, de Galdeano E. Evaluation of the effectiveness of a Mindfulness and Self-Compassion program to reduce stress and prevent burnout in Primary Care health professionals. Aten Primaria. 2018; 50(3):141-50. https://doi.org/10.1016/j.aprim. 2017.03.009 PMID: 28629886

85. Barrett B, Hayney MS, Muller D, Rakel D, Ward A, Obasi CN, et al. Meditation or exercise for preventing acute respiratory infection: a randomized controlled trial. Ann Fam Med. 2012; 10(4):337-46. Epub 2012/07/11. https://doi.org/10.1370/afm.1376 PMID: 22778122.

86. Barrett B, Rakel D, Hayney M, Muller D, Zgierska A, Obasi C, et al. P02.36. Meditation or exercise for preventing acute respiratory infection: a randomized controlled trial. BMC Complement Altern Med. 2012; 12(Suppl 1):1-. Language: English. Entry Date: 20121129. Revision Date: 20150711. Publication Type: Journal Article.

87. Obasi CN, Brown R, Ewers T, Barlow S, Gassman M, Zgierska A, et al. Advantage of meditation over exercise in reducing cold and flu illness is related to improved function and quality of life. Influenza Other Respi Viruses. 2013; 7(6):938-44. http://dx.doi.org/10.1111/irv.12053. PMID: 23170828.

88. Zgierska A, Obasi C, Brown R, Ewers T, Rabago D, Barrett B. P02.57. Mindfulness meditation versus exercise in the prevention of acute respiratory infection, possible mechanisms of action: a randomized controlled trial. BMC Complement Altern Med. 2012; 12(Suppl 1):1-. https://doi.org/10.1186/14726882-12-s1-p113 PMID: 104481481. Language: English. Entry Date: 20121129. Revision Date: 20150711. Publication Type: Journal Article.

89. Zgierska A, Obasi CN, Brown R, Ewers T, Muller D, Gassman M, et al. Randomized Controlled Trial of Mindfulness Meditation and Exercise for the Prevention of Acute Respiratory Infection: Possible Mechanisms of Action. Evidence-based Complementary \& Alternative Medicine (eCAM). 2013; 2013:1-14. 2013/952716. https://doi.org/10.1155/2013/952716 PMID: 24191174. Language: English. Entry Date: 20140616. Revision Date: 20150710. Publication Type: Journal Article.

90. Hayney MS, Coe CL, Muller D, Obasi CN, Backonja U, Ewers T, et al. Age and psychological influences on immune responses to trivalent inactivated influenza vaccine in the meditation or exercise for preventing acute respiratory infection (MEPARI) trial. Hum Vaccin Immunother. 2014; 10(1):2759-67. http://dx.doi.org/10.4161/hv.26661. PMID: 24096366.

91. Rakel D, Mundt M, Ewers T, Fortney L, Zgierska A, Gassman M, et al. Value associated with mindfulness meditation and moderate exercise intervention in acute respiratory infection: the MEPARI Study. Fam Pract. 2013; 30(4):390-7. Epub 2013/03/22. https://doi.org/10.1093/fampra/cmt008 PMID: 23515373.

92. Barrett B, Hayney MS, Muller D, Rakel D, Brown R, Zgierska AE, et al. Meditation or exercise for preventing acute respiratory infection (MEPARI-2): A randomized controlled trial. PLoS ONE. 2018; 13 (6):e0197778. http://dx.doi.org/10.1371/journal.pone.0197778. PMID: 29933369.

93. Maxwell L, Barrett B, Chase J, Brown R, Ewers T. Self-reported mental health predicts acute respiratory infection. Wis Med J. 2015; 114(3):100-4. PMID: 26273187.

94. Hayney MS, Henriquez KM, Barnet JH, Ewers T, Champion HM, Flannery S, et al. Serum IFN-Yinduced protein $10(\mathrm{IP}-10)$ as a biomarker for severity of acute respiratory infection in healthy adults. $J$ Clin Virol. 2017; 90:32-7. https://doi.org/10.1016/j.jcv.2017.03.003 PMID: 28334685 
95. Meyer JD, Torres ER, Grabow ML, Zgierska AE, Teng HY, Coe CL, et al. Benefits of 8-wk Mindfulness-based Stress Reduction or Aerobic Training on Seasonal Declines in Physical Activity. Med Sci Sports Exerc. 2018; 50 (9):1850-8. https://doi.org/10.1249/MSS.0000000000001636 PMID: 30113538

96. Barrett B. Predictors of Mindfulness Meditation and Exercise Practice, from MEPARI-2, a Randomized Controlled Trial. Mind. 2019; 10:1842-54. https://doi.org/10.1007/s12671-019-01137-3 PMID: 31938076

97. Meyer JD, Hayney MS, Coe CL, Ninos CL, Barrett BP, et al. J Sport Exerc Psychol. 2019; 41 (2). https://journals.humankinetics.com/view/journals/jsep/41/2/article-p96.xml

98. Beattie J, Hall H, Biro MA, East C, Lau R. Effects of mindfulness on maternal stress, depressive symptoms and awareness of present moment experience: A pilot randomised trial. Midwifery. 2017; 50:174-83. https://doi.org/10.1016/j.midw.2017.04.006 PMID: 28463789. Language: English. Entry Date: 20170606. Revision Date: 20170606. Publication Type: Article.

99. Behbahani M, Zargar F, Assarian F, Akbari H. Effects of mindful parenting training on clinical symptoms in children with attention deficit hyperactivity disorder and parenting stress: Randomized controlled trial. Iranian Journal of Medical Sciences. 2018; 43 (6):596-604. PMID: 30510336

100. Benn R, Akiva T, Arel S, Roeser RW. Mindfulness Training Effects for Parents and Educators of Children With Special Needs. Dev Psychol. 2012; 48(5):1476-87. https://doi.org/10.1037/a0027537 PMID: 22409766. Language: English. Entry Date: 20120905. Revision Date: 20150712. Publication Type: Journal Article.

101. Berghmans $C$, Tarquinio $C$, Kretsch $M$. Impact of the therapeutic approach of mindfulness-based stress reduction (MBSR) on psychic health (stress, anxiety, depression) in students: A controlled and randomized pilot study. Journal de Therapie Comportementale et Cognitive. 2010; 20 (1):11-5. https://doi.org/10.1016/j.jtcc.2010.03.001

102. Black D, Luders E, Breen E, Olmstead R, Michael I. Mindfulness Training Versus Sleep Hygiene for Insomnia Symptoms in Older Adults: A Randomized Controlled Comparison Trial. J Altern Complement Med. 2014; 20(5):A14-5. https://doi.org/10.1089/acm.2014.5034.abstract PMID: 103939607. Language: English. Entry Date: 20140512. Revision Date: 20150710. Publication Type: Journal Article.

103. Black DS, Luders E, Breen E, Olmstead R, Irwin M. Mindfulness Training Versus Sleep Hygiene for Insomnia Symptoms in Older Adults: A Randomized Controlled Comparison Trial. Ann Behav Med. 2014; 47:S135-S.

104. Black DS, Luders E, Olmstead R, Irwin MR. Effect of Mindfulness Training on Sleep, Inflammatory Transcription, and Brain Morphology: A Randomized Comparison Trial with Older Adults. Psychosom Med. 2014; 76(3):A28-A.

105. Black DS, O'Reilly GA, Olmstead R, Breen EC, Irwin MR. Mindfulness-based intervention for prodromal sleep disturbances in older adults: Design and methodology of a randomized controlled trial. Contemp Clin Trials. 2014; 39(1):22-7. http://dx.doi.org/10.1016/j.cct.2014.06.013. PMID: 24993561.

106. Black DS, O'Reilly GA, Olmstead R, Breen EC, Irwin MR. Mindfulness Meditation and Improvement in Sleep Quality and Daytime Impairment Among Older Adults With Sleep Disturbances: A Randomized Clinical Trial. JAMA Intern Med. 2015; 175(4):494-501. Epub 2015/02/17. https://doi.org/10.1001/ jamainternmed.2014.8081 PMID: 25686304.

107. Brown KW, Coogle CL, Wegelin J. A pilot randomized controlled trial of mindfulness-based stress reduction for caregivers of family members with dementia. Aging Ment Health. 2016; 20(11):1157-66. Epub 2015/07/28. https://doi.org/10.1080/13607863.2015.1065790 PMID: 26211415.

108. Carmody JF, Crawford S, Salmoirago-Blotcher E, Leung K, Churchill L, Olendzki N. Mindfulness training for coping with hot flashes: results of a randomized trial. Menopause. 2011; 18(6):611-20. Epub 2011/03/05. https://doi.org/10.1097/gme.0b013e318204a05c PMID: 21372745.

109. Carson JW, Carson KM, Gil KM, Baucom DH. Mindfulness-based relationship enhancement. Behav Ther. 2004; 35(3):471-94. http://dx.doi.org/10.1016/S0005-7894\%2804\%2980028-5. PMID: 40050935.

110. Carson JW. Mindfulness meditation-based treatment for relationship enhancement. Ann Arbor: The University of North Carolina at Chapel Hill; 2002.

111. Cerna C, Garcia FE, Tellez A. Brief mindfulness, mental health, and cognitive processes: A randomized controlled trial. PsyCh Journal. 2019; 9(359-69). https://doi.org/10.1002/pchj.325 PMID: 31805614

112. Christopher MS, Hunsinger M, Goerling LRJ, Bowen S, Rogers BS, Gross CR, et al. Mindfulnessbased resilience training to reduce health risk, stress reactivity, and aggression among law enforcement officers: A feasibility and preliminary efficacy trial. Psychiatry Res. 2018; 264:104-15. https://doi. org/10.1016/j.psychres.2018.03.059 PMID: 29627695 
113. Hunsinger M, Christopher M, Schmidt AM. Mindfulness Training, Implicit Bias, and Force Response Decision-Making. Mind. 2019; 10:2555-66. https://doi.org/10.1007/s12671-019-01213-8.

114. Ribeiro L, Colgan DD, Hoke CK, Hunsinger M, Bowen S, Oken BS, et al. Differential Impact of Mindfulness Practices on Aggression Among Law Enforcement Officers. Mindfulness. 2020; 11:734-45. https://doi.org/10.1007/s12671-019-01289-2.

115. Cohen-Katz J, Wiley SD, Capuano T, Baker DM, Shapiro S. The effects of mindfulness-based stress reduction on nurse stress and burnout: a quantitative and qualitative study. . . part of a series. Holist Nurs Pract. 2004; 18(6):302-8. https://doi.org/10.1097/00004650-200411000-00006 PMID: 15624277. Language: English. Entry Date: 20050107. Revision Date: 20150818. Publication Type: Journal Article.

116. Cohen-Katz J, Wiley SD, Capuano T, Baker DM, Shapiro S. The effects of mindfulness-based stress reduction on nurse stress and burnout, part II: a quantitative and qualitative study. Holist Nurs Pract. 2005; 19(1):26-35. https://doi.org/10.1097/00004650-200501000-00008 PMID: 15736727. Language: English. Entry Date: 20051118. Revision Date: 20150818. Publication Type: Journal Article.

117. Corsica J, Hood MM, Katterman S, Kleinman B, Ivan I. Development of a novel mindfulness and cognitive behavioral intervention for stress-eating: a comparative pilot study. Eat Behav. 2014; 15 (4):694-9. https://doi.org/10.1016/j.eatbeh.2014.08.002 PMID: 25462029. Language: English. Entry Date: 20150821. Revision Date: 20150923. Publication Type: Journal Article

118. Cousin $\mathrm{G}$, Crane $\mathrm{C}$. Changes in disengagement coping mediate changes in affect following mindfulness-based cognitive therapy in a non-clinical sample. Br J Psychol. 2016; 107(3):434-47. https:// doi.org/10.1111/bjop.12153 PMID: 26385256. Language: English. Entry Date: 20160712. Revision Date: 20180627. Publication Type: Article.

119. Damião Neto A, Lucchetti ALG, da Silva EO, Lucchetti G. Effects of a Required Large-Group Mindfulness Meditation Course on First-Year Medical Students' Mental Health and Quality of Life: a Randomized Controlled Trial. J Gen Intern Med. 2019. https://doi.org/10.1007/s11606-019-05284-0 PMID: 31452038

120. Davidson RJ, Kabat-Zinn J, Schumacher J, Rosenkrnz M, Muller D, Santorelli SF, et al. Alterations in brain and immune function produced by mindfulness meditation. Psychosom Med. 2003; 65(4):56470. https://doi.org/10.1097/01.psy.0000077505.67574.e3 PMID: 12883106. Language: English. Entry Date: 20060331. Revision Date: 20150711. Publication Type: Journal Article.

121. Delgado LC, Guerra P, Perakakis P, Vera MN, Reyes del Paso G, Vila J. Treating chronic worry: Psychological and physiological effects of a training programme based on mindfulness. Behav Res Ther. 2010; 48(9):873-82. https://dx.doi.org/10.1016/j.brat.2010.05.012. PMID: 20541180.

122. Delgado-Pastor LC, Ciria LF, Blanca B, Mata JL, Vera MN, Vila J. Dissociation between the cognitive and interoceptive components of mindfulness in the treatment of chronic worry. J Behav Ther Exp Psychiatry. 2015; 48:192-9. Epub 2015/04/29. https://doi.org/10.1016/j.jbtep.2015.04.001 PMID: 25912677.

123. Desbordes G, Negi LT, Pace TWW, Alan Wallace B, Raison CL, Schwartz EL. Effects of mindfulattention and compassion meditation training on amygdala response to emotional stimuli in an ordinary, Nonmeditative State. Front Hum Neurosci. 2012; 6:292. http://dx.doi.org/10.3389/fnhum.2012. 00292. PMID: 23125828.

124. De Vibe M, Solhaug I, Tyssen R, Friborg O, Rosenvinge JH, Sørlie T. Mindfulness training for stress management: a randomised controlled study of medical and psychology students. BMC Med Educ. 2013; 13 (1):107. https://doi.org/10.1186/1472-6920-13-107 PMID: 23941053

125. Halland E, de Vibe M, Solhaug I, Friborg O, Rosenvinge JH, Tyssen R, et al. Mindfulness Training Improves Problem-Focused Coping in Psychology and Medical Students: Results from a Randomized Controlled Trial. Coll Stud J. 2015; 49 (3):387-98.

126. de Vibe M, Solhaug I, Tyssen $\mathrm{R}$, Friborg O, Rosenvinge JH, Sorlie T, et al. Does Personality Moderate the Effects of Mindfulness Training for Medical and Psychology Students? Mind. 2015; 6(2):281-9.

127. Solhaug I, de Vibe M, Friborg O, Sørlie T, Tyssen R, Bjørndal A, et al. Long-term Mental Health Effects of Mindfulness Training: a 4-Year Follow-up Study. Mind. 2019; 10 (8):1661-72. https://doi. org/10.1007/s12671-019-01100-2

128. Hanley AW, de Vibe M, Solhaug I, Gonzalez-Pons K, Garland EL. Mindfulness training reduces neuroticism over a 6-year longitudinal randomized control trial in Norwegian medical and psychology students. J Res Pers. 2019; 82:103859. https://doi.org/10.1016/j.jrp.2019.103859

129. Duncan LG, Cohn MA, Chao MT, Cook JG, Riccobono J, Bardacke N. Benefits of preparing for childbirth with mindfulness training: a randomized controlled trial with active comparison. BMC Pregnancy Childbirth. 2017; 17:1-11. Language: English. Entry Date: In Process. Revision Date: 20180322. Publication Type: journal article. 
130. Dvorakova K, Kishida M, Li J, Elavsky S, Broderick PC, Agrusti MR, et al. Promoting healthy transition to college through mindfulness training with first-year college students: Pilot randomized controlled trial. Journal of American college health: J of ACH. 2017; 65(4):259-67. Epub 2017/01/12. https://doi. org/10.1080/07448481.2017.1278605 PMID: 28076182.

131. Dykens EM, Taylor JL, Fisher MH, Lambert W, Miodrag N. Reducing Distress in Mothers of Children With Autism and Other Disabilities: A Randomized Trial. Pediatrics. 2014; 134 (2):e454 -e63. https:// doi.org/10.1542/peds.2013-3164 PMID: 25049350

132. Dziok MD. Mindfulness meditation for psychological distress. Ann Arbor: Yeshiva University; 2010.

133. Esch T, Winkler J, Auwarter V, Gnann H, Huber R, Schmidt S. Neurobiological aspects of mindfulness in pain autoregulation: Unexpected results from a randomized-controlled trial and possible implications for meditation research. Front Hum Neurosci. 2017; 10(674). http://dx.doi.org/10.3389/fnhum. 2016.00674. PMID: 28184192

134. Ferraioli SJ, Harris SL. Comparative effects of mindfulness and skills-based parent training programs for parents of children with autism: Feasibility and preliminary outcome data. Mind. 2013; 4(2):89-101. https://doi.org/10.1007/s12671-012-0099-0 2013-15611-002.

135. Fiocco AJ, Mallya S, Farzaneh M, Koszycki D. Exploring the benefits of mindfulness training in healthy community-dwelling older adults: A randomized controlled study using a mixed methods approach. Mind. 2018; 10(4):737-48. https://doi.org/10.1007/s12671-018-1041-x 2018-52326-001.

136. Flook L, Goldberg SB, Pinger L, Bonus K, Davidson RJ. Mindfulness for Teachers: A Pilot Study to Assess Effects on Stress, Burnout, and Teaching Efficacy. Mind Brain Educ. 2013; 7 (3):182-95.

137. Frisvold MH. The 'midlife study': Mindfulness as an intervention to change health behaviors in midlife women: University of Minnesota; 2009.

138. Turner L, Galante J, Vainre M, Stochl J, Dufour G, Jones P. Immune dysregulation among students exposed to exam stress and its mitigation by mindfulness training: findings from an exploratory randomised trial. Sci Rep. 2020; 10 (1):1-11. https://doi.org/10.17863/CAM.49516 PMID: 31913322

139. Bóo SJM, Childs-Fegredo J, Cooney S, Datta B, Dufour G, Jones PB, et al. A follow-up study to a randomised control trial to investigate the perceived impact of mindfulness on academic performance in university students. Couns Psychother Res. 2019; 20 (2):286-301. https://doi.org/10.1002/capr. 12282

140. Gallego J, Aguilar-Parra JM, Cangas AJ, Langer Al, Manas I. Effect of a mindfulness program on stress, anxiety and depression in university students. Span J Psychol. 2014; 17:E109. https://doi.org/ 10.1017/sjp.2014.102 PMID: 26055051.

141. Gambrel LE, Piercy FP. Mindfulness-based relationship education for couples expecting their first child - part 1: a randomized mixed-methods program evaluation. J Marital Fam Ther. 2015; 41(1):524. https://doi.org/10.1111/jmft.12066 PMID: 24433518. Language: English. Entry Date: 20150923. Revision Date: 20151007. Publication Type: journal article. Journal Subset: Biomedical.

142. Gambrel LE. The Mindful Transition to Parenthood Program: Developing and Evaluating a Psychoeducational-Experiential Intervention for Couples Expecting Their First Child. Ann Arbor: Virginia Polytechnic Institute and State University; 2012.

143. Giannandrea A, Simione L, Pescatori B, Ferrell K, Olivetti Belardinelli M, Hickman SD, et al. Effects of the mindfulness-based stress reduction program on mind wandering and dispositional mindfulness facets. Mind. 2018; 10(1):185-95. https://doi.org/10.1007/s12671-018-1070-5 2018-61307-001.

144. Glass CR, Spears CA, Perskaudas R, Kaufman KA. Mindful Sport Performance Enhancement: Randomized Controlled Trial of a Mental Training Program With Collegiate Athletes. J Clin Sport Psychol. 2019; 13(4):609-28. https://doi.org/10.1123/jcsp.2017-0044

145. Grandpierre Z. Feasibility Study: Can Mindfulness Practice Benefit Executive Function and Improve Academic Performance? Ann Arbor: University of Ottawa (Canada); 2013.

146. Greenberg J, Reiner K, Meiran N. "Mind the Trap": Mindfulness Practice Reduces Cognitive Rigidity. PLoS ONE. 2010; 5 (1):e36206. https://doi.org/10.1371/journal.pone.0036206 PMID: 22615758

147. Greenberg J, Reiner K, Meiran N. 'Off with the old': Mindfulness practice improves backward inhibition. Front Psychol. 2013; 3:618. https://doi.org/10.3389/fpsyg.2012.00618 PMID: 23335909 201308328-001.

148. Greeson JM, Juberg MK, Maytan M, James K, Rogers H. A randomized controlled trial of Koru: a mindfulness program for college students and other emerging adults. Journal of American college health: J of ACH. 2014; 62(4):222-33. https://doi.org/10.1080/07448481.2014.887571 PMID: 24499130.

149. Guardino CM, Dunkel Schetter C, Bower JE, Lu MC, Smalley SL. Randomised controlled pilot trial of mindfulness training for stress reduction during pregnancy. Psychol Health. 2014; 29(3):334-49. 
https://doi.org/10.1080/08870446.2013.852670 PMID: 24180264. Language: English. Entry Date: 20140130. Revision Date: 20150824. Publication Type: Journal Article.

150. Haarig F, Winkler D, Graubner M, Sipos L, Muhlig S. Mindfulness for stress management-Results of a randomized controlled pilot trial of mindfulness stress-reduction-training (MST). [German] Achtsamkeit zur Stressbewaltigung: Ergebnisse einer randomisiert-kontrollierten Pilotstudie zu einem Achtsamkeitsorientierten Stressbewaltigungstraining (AST). Zeitschrift fur Psychiatrie, Psychologie und Psychotherapie. 2016; 64(3):187-97. http://dx.doi.org/10.1024/1661-4747/a000278. PMID: 611431217.

151. Hou RJ, Wong SYS, Yip BHK, Hung ATF, Lo HHM, Chan PHS, et al. The effects of mindfulnessbased stress reduction program on the mental health of family caregivers: A randomized controlled trial. Psychother Psychosom. 2013; 83(1):45-53. http://dx.doi.org/10.1159/000353278. PMID: 24281411.

152. Hou J. The Effects of Mindfulness-based Stress Reduction Program on Depressive Symptoms Reduction among Family Caregivers in Hong Kong. Ann Arbor: The Chinese University of Hong Kong (Hong Kong); 2012.

153. Huang SL, Li RH, Huang FY, Tang FC. The potential for mindfulness-based intervention in workplace mental health promotion: Results of a randomized controlled trial. PloS ONE. 2015; 10:e0138089. http://dx.doi.org/10.1371/journal.pone.0138089. PMID: 26367270.

154. Hunt M, Al-Braiki F, Dailey S, Russell R, Simon K. Mindfulness Training, Yoga, or Both? Dismantling the Active Components of a Mindfulness-Based Stress Reduction Intervention. Mind. 2018; 9(2):512 20. https://doi.org/10.1007/s12671-017-0793-z

155. Hwang Y-S, Goldstein H, Medvedev ON, Singh NN, Noh J-E, Hand K. Mindfulness-based intervention for educators: Effects of a school-based cluster randomized controlled study. Mind. 2019; 10 (7):1417-36. https://doi.org/10.1007/s12671-019-01147-1 2019-18008-001.

156. Ireland MJ, Clough B, Gill K, Langan F, O'Connor A, Spencer L. A randomized controlled trial of mindfulness to reduce stress and burnout among intern medical practitioners. Med Teach. 2017; 39 (4):409-14. https://doi.org/10.1080/0142159X.2017.1294749 PMID: 28379084. Language: English. Entry Date: 20170412. Revision Date: 20170419. Publication Type: Article.

157. Isbel B, Summers MJ. Distinguishing The Cognitive Processes Of Mindfulness: Developing A Standardised Mindfulness Technique For Use In Longitudinal Randomised Control Trials. Conscious Cogn. 2017; 52:75-92. https://doi.org/10.1016/j.concog.2017.04.019. PMID: 28499257

158. Isbel B, Lagopoulos J, Hermens DF, Summers MJ. Mindfulness induces changes in anterior alpha asymmetry in healthy older adults. Mind. 2019; 10(7):1381-94. https://doi.org/10.1007/s12671-01901106-w 2019-07993-001.

159. Isbel BD, Lagopoulos J, Hermens DF, Summers MJ. Mental training affects electrophysiological markers of attention resource allocation in healthy older adults. Neurosci Lett. 2019;698:186-91. http://dx. doi.org/10.1016/j.neulet.2019.01.029 PMID: 30659914

160. Jain S, Shapiro SL, Swanick S, Roesch SC, Mills PJ, Bell I, et al. A randomized controlled trial of mindfulness meditation versus relaxation training: effects on distress, positive states of mind, rumination, and distraction. Ann Behav Med. 2007; 33 (1):11-21. https://doi.org/10.1207/s15324796abm3301 2 PMID: 17291166

161. James K, Rimes KA. Mindfulness-based cognitive therapy versus pure cognitive behavioural selfhelp for perfectionism: A pilot randomised study. Mind. 2018; 9(3):801-14. https://doi.org/10.1007/ s12671-017-0817-8 PMID: 29875882 2017-46873-001.

162. Josefsson $T$, Lindwall $M$, Broberg $A G$. The effects of a short-term mindfulness based intervention on self-reported mindfulness, decentering, executive attention, psychological health, and coping style: Examining unique mindfulness effects and mediators. Mind. 2014; 5(1):18-35. https://doi.org/10. 1007/s12671-012-0142-12014-04440-003.

163. Kang YS, Choi SY, Ryu E. The effectiveness of a stress coping program based on mindfulness meditation on the stress, anxiety, and depression experienced by nursing students in Korea. Nurse Educ Today. 2009; 29(5):538-43. https://doi.org/10.1016/j.nedt.2008.12.003 PMID: 19141364. Language: English. Entry Date: 20090807. Revision Date: 20150819. Publication Type: Journal Article.

164. Kaviani A, Hatami N, ShafiAbadi A. The impact of mindfulness-based cognitive therapy on the quality of life in non-clinically depressed people. Advances in Cognitive Science. 2008; 10(4):97-. 200922379-004.

165. Kaviani H, Hatami N, Javaheri F. The impact of Mindfulness-Based Cognitive Therapy (MBCT) on mental health and quality of life in a sub-clinically depressed population. Psychoterapia. 2012; 1:218. PMID: 364824049.

166. Kingston J, Chadwick $P$, Meron $D$, Skinner TC. A pilot randomized control trial investigating the effect of mindfulness practice on pain tolerance, psychological well-being, and physiological activity. $J$ 
Psychosom Res. 2007; 62(3):297-300. Epub 2007/02/28. https://doi.org/10.1016/j.jpsychores.2006. 10.007 PMID: 17324679.

167. Kirk U, Gu X, Sharp C, Hula A, Fonagy P, Montague PR. Mindfulness training increases cooperative decision making in economic exchanges: Evidence from fMRI. Neuroimage. 2016; 138:274-83. https://dx.doi.org/10.1016/j.neuroimage.2016.05.075. PMID: 27266443.

168. Kirk U, Pagnoni G, Hétu S, et al. Short-term mindfulness practice attenuates reward prediction errors signals in the brain. Sci Rep. 2019; 9.

169. Klatt MD, Sieck C, Gascon G, Malarkey W, Huerta T. A healthcare utilization cost comparison between employees receiving a worksite mindfulness or a diet/exercise lifestyle intervention to matched controls 5 years post intervention. Complement Ther Med. 2016; 27:139-44. Epub 2016/08/ 16. https://doi.org/10.1016/j.ctim.2016.05.008 PMID: 27515889.

170. Klatt M, Steinberg B, Marks D, Duchemin A. OA04.04. Changes in physiological and psychological markers of stress in hospital personnel after a low-dose mindfulness-based worksite intervention. BMC Complement Altern Med. 2012; 12(Suppl 1):1-. https://doi.org/10.1186/1472-6882-12-s1-016 PMID: 104481319. Language: English. Entry Date: 20121129. Revision Date: 20150711. Publication Type: Journal Article.

171. Kor PPK, Liu JYW, Chien WT. Effects of a modified mindfulness-based cognitive therapy for family caregivers of people with dementia: A pilot randomized controlled trial. Int J Nurs Stud. 2019; 98:10717. http://dx.doi.org/10.1016/j.ijnurstu.2019.02.020 PMID: 30922609

172. Krick A, Felfe J. Who benefits from mindfulness? The moderating role of personality and social norms for the effectiveness on psychological and physiological outcomes among police officers. J Occup Health Psychol. 2019; 25(2):99. https://doi.org/10.1037/ocp0000159 PMID: 31219270 2019-33410001.

173. Kuhlmann SM, Huss M, Burger A, Hammerle F. Coping with stress in medical students: results of a randomized controlled trial using a mindfulness-based stress prevention training (MediMind) in Germany. BMC Med Educ. 2016; 16(1):316. Epub 2016/12/30. https://doi.org/10.1186/s12909-016-08338 PMID: 28031044.

174. Kuhlmann SM, Burger A, Esser G, Hammerle F. A mindfulness-based stress prevention training for medical students (MediMind): study protocol for a randomized controlled trial. Trials. 2015; 16:40. Epub 2015/04/19. https://doi.org/10.1186/s13063-014-0533-9 PMID: 25887430.

175. Lacerda SS, Little SW, Kozasa EH. A Stress Reduction Program Adapted for the Work Environment: A Randomized Controlled Trial With a Follow-Up. Front Psychol. 2018; 9:668. https://doi.org/10.3389/ fpsyg.2018.00668 PMID: 29867646

176. Lara-Cinisomo S, Fujimoto EM, Santens RL. Feasibility of a Mindfulness-Based Intervention for Caregivers of Veterans: A Pilot Study. Journal of holistic nursing: official journal of the American Holistic Nurses' Association. 2019; 37(4):322-37. http://dx.doi.org/10.1177/0898010119831580. PMID: 30862225

177. Lara-Cinisomo S, Santens RL, Fujimoto EM. A pilot randomized controlled trial of a mindfulnessbased intervention for caregivers of veterans. Mind. 2019. https://doi.org/10.1177/ 0898010119831580 PMID: 30862225 2019-18203-001.

178. Lebares CC, Hershberger AO, Guvva EV, Desai A, Mitchell J, Shen W, et al. Feasibility of Formal Mindfulness-Based Stress-Resilience Training among Surgery Interns: A Randomized Clinical Trial. JAMA Surg. 2018; 153 (10):e182734. http://dx.doi.org/10.1001/jamasurg.2018.2734 PMID: 30167655

179. Lebares CC, Guvva EV, Olaru M, Sugrue LP, Staffaroni AM, Delucchi KL, et al. Efficacy of mindfulness-based cognitive training in surgery additional analysis of the mindful surgeon pilot randomized clinical trial. JAMA Netw Open. 2019; 2 (5):e194108. http://dx.doi.org/10.1001/jamanetworkopen. 2019.4108 PMID: 31125095

180. Lee WK, Bang HJ. The effects of mindfulness-based group intervention on the mental health of middle-aged Korean women in community. Stress Health. 2010; 26 (4):341-8. https://doi.org/10.1002/ smi.1303

181. Li YY, Liu F, Zhang Q, Liu XH, Wei P. The Effect of Mindfulness Training on Proactive and Reactive Cognitive Control. Front Psychol. 2018; 9:1002. https://doi.org/10.3389/fpsyg.2018.01002 PMID: 29973897

182. Lin L, He G, Yan J, Gu C, Xie J. The Effects of a Modified Mindfulness-Based Stress Reduction Program for Nurses: A Randomized Controlled Trial. Workplace health \& safety. 2019; 67 (3):111-22. http://dx.doi.org/10.1177/2165079918801633 PMID: 30370837

183. Liu X-H, Xu W, Wang Y-Z, Liu H-H. Effect of mindfulness training on subjective well-being in volunteers: A six-week randomized controlled trial. Chin Ment Health J. 2013; 27(8):597-601. 201330370-008. 
184. Liu X, Xu W, Wang Y, Williams JM, Geng Y, Zhang Q. Can Inner Peace be Improved by Mindfulness Training: A Randomized Controlled Trial. Stress and health: journal of the International Society for the Investigation of Stress. 2015; 31(3):245-54. Epub 2013/11/23. https://doi.org/10.1002/smi.2551 PMID: 24265118.

185. Lo HHM, Chan SKC, Szeto MP, Chan CYH, Choi CW. A Feasibility Study of a Brief MindfulnessBased Program for Parents of Preschool Children with Developmental Disabilities. Mind. 2017; 8 (6):1665-73. https://doi.org/10.1007/s12671-017-0741-y

186. Lonnberg G, Jonas W, Unternaehrer E, Branstrom R, Nissen E, Niemi M. Effects of a mindfulness based childbirth and parenting program on pregnant women's perceived stress and risk of perinatal depression-Results from a randomized controlled trial. J Affect Disord. 2020; 262:133-42. http://dx. doi.org/10.1016/j.jad.2019.10.048 PMID: 31733457

187. Lönnberg G, Jonas W, Bränström R, Nissen E, Niemi M. Long-term effects of a mindfulness-based childbirth and parenting program —a randomized controlled trial. Mindfulness. 2020. https://doi.org/10. 1007/s12671-020-01403-9 2020-32931-001.

188. Lopez-Maya E, Olmstead R, Irwin MR. Mindfulness meditation and improvement in depressive symptoms among Spanish-And English speaking adults: A randomized, controlled, comparative efficacy trial. PLoS ONE. 2019; 14 (7):e0219425. http://dx.doi.org/10.1371/journal.pone.0219425 PMID: 31276540

189. Lynch S, Gander ML, Nahar A, Kohls N, Walach H. Mindfulness-Based Coping With University Life: A Randomized Wait-List Controlled Study. SAGE Open. 2018; 8 (1).

190. Ma DY. Mindfulness-based cognitive therapy for community-dwelling adults with anxiety symptoms: An exploration of efficacy and cognitive mechanism. 2019; 80. 2019-41128-294.

191. MacCoon DG, Imel ZE, Rosenkranz MA, Sheftel JG, Weng HY, Sullivan JC, et al. The validation of an active control intervention for Mindfulness Based Stress Reduction (MBSR). Behav Res Ther. 2012; 50(1):3-12. http://dx.doi.org/10.1016/j.brat.2011.10.011. PMID: 22137364.

192. Rosenkranz MA, Davidson RJ, Maccoon DG, Sheridan JF, Kalin NH, Lutz A. A comparison of mindfulness-based stress reduction and an active control in modulation of neurogenic inflammation. Brain Behav Immun. 2013; 27(1):174-84. Epub 2012/10/25. https://doi.org/10.1016/j.bbi.2012.10.013 PMID: 23092711.

193. MacCoon DG, MacLean KA, Davidson RJ, Saron CD, Lutz A. No sustained attention differences in a longitudinal randomized trial comparing mindfulness based stress reduction versus active control. PLoS ONE. 2014; 9(6):e97551. http://dx.doi.org/10.1371/journal.pone.0097551. PMID: 24955584.

194. Goldberg SB, Wielgosz J, Dahl C, Schuyler B, MacCoon DS, Rosenkranz M, et al. Does the Five Facet Mindfulness Questionnaire measure what we think it does? Construct validity evidence from an active controlled randomized clinical trial. Psychol Assess. 2016; 28(8):1009-14. https://doi.org/10. 1037/pas0000233 PMID: 26460893. Language: English. Entry Date: 20180719. Revision Date: 20180413. Publication Type: journal article.

195. Malarkey WB, Jarjoura D, Klatt M. Workplace based mindfulness practice and inflammation: a randomized trial. Brain Behav Immun. 2013; 27(1):145-54. Epub 2012/10/20. https://doi.org/10.1016/j. bbi.2012.10.009 PMID: 23078984.

196. Malinowski P, Moore AW, Mead BR, Gruber T. Mindful Aging: The Effects of Regular Brief Mindfulness Practice on Electrophysiological Markers of Cognitive and Affective Processing in Older Adults. Mind. 2017; 8(1):78-94. https://dx. doi.org/10.1007/s12671-015-0482-8. PMID: 28163795.

197. Moore AW. Facilitating healthy ageing: neuroprotective effects of mindfulness practice. Ann Arbor: Liverpool John Moores University (United Kingdom); 2013.

198. Manotas M, Segura C, Eraso M, Oggins J, McGovern K. Association of brief mindfulness training with reductions in perceived stress and distress in Colombian health care professionals. Int J Stress Manag. 2014; 21 (2):207-25. https://doi.org/10.1037/a0035150

199. Manotas MA. Brief mindfulness training to improve mental health with colombian healthcare professionals. US: ProQuest Information \& Learning; 2013.

200. Moody K, Kramer D, Santizo RO, Magro L, Wyshogrod D, Ambrosio J, et al. Helping the Helpers: Mindfulness Training for Burnout in Pediatric Oncology-A Pilot Program. J Pediatr Oncol Nurs. 2013; 30 (5):275-84. https://doi.org/10.1177/1043454213504497 PMID: 24101747

201. Moritz S, Quan H, Rickhi B, Liu M, Angen M, Vintila R, et al. A home study-based spirituality education program decreases emotional distress and increases quality of life-A randomized, controlled trial. Altern Ther Health Med. 2006; 12(6):26-35. PMID: 17131979.

202. Moynihan JA, Chapman BP, Klorman R, Krasner MS, Duberstein PR, Brown KW, et al. Mindfulnessbased stress reduction for older adults: effects on executive function, frontal alpha asymmetry and immune function. Neuropsychobiology. 2013; 68(1):34-43. Epub 2013/06/19. https://doi.org/10.1159/ 000350949 PMID: 23774986. 
203. Gallegos AM, Hoerger M, Talbot NL, Krasner MS, Knight JM, Moynihan JA, et al. Toward Identifying the Effects of the Specific Components of Mindfulness-Based Stress Reduction on Biologic and Emotional Outcomes Among Older Adults. J Altern Complement Med. 2013; 19(10):787-92. https://doi. org/10.1089/acm.2012.0028 PMID: 23383976. Language: English. Entry Date: 20131018. Revision Date: 20150820. Publication Type: Journal Article.

204. Gallegos AM, Hoerger M, Talbot NL, Moynihan JA, Duberstein PR. Emotional benefits of mindfulness-based stress reduction in older adults: the moderating roles of age and depressive symptom severity. Aging Ment Health. 2013; 17(7):823-9. Epub 2013/05/24. https://doi.org/10.1080/13607863. 2013.799118 PMID: 23697871.

205. Visser PL, Hirsch JK, Brown KW, Ryan R, Moynihan JA. Components of Sleep Quality as Mediators of the Relation Between Mindfulness and Subjective Vitality Among Older Adults. Mind. 2015; 6 (4):723-31.

206. Barkan T, Hoerger M, Gallegos AM, Turiano NA, Duberstein PR, Moynihan JA. Personality Predicts Utilization of Mindfulness-Based Stress Reduction During and Post-Intervention in a Community Sample of Older Adults. J Altern Complement Med. 2016; 22(5):390-5. https://doi.org/10.1089/acm. 2015.0177 PMID: 27031734. Language: English. Entry Date: 20160506. Revision Date: 20170501. Publication Type: Article.

207. Gallegos AM, Moynihan J, Pigeon WR. A Secondary Analysis of Sleep Quality Changes in Older Adults From a Randomized Trial of an MBSR Program. J Appl Gerontol. 2018; 37 (11):1327-43. https://doi.org/10.1177/0733464816663553 PMID: 27511921

208. Elliot A, Gallegos AM, Moynihan JA, Chapman BP. Associations of mindfulness with depressive symptoms and well-being in older adults: the moderating role of neuroticism. Aging Ment Health. 2019; 23 (4). https://doi.org/10.1080/13607863.2017.1423027 PMID: 29338322

209. Mrazek MD, Franklin MS, Phillips DT, Baird B, Schooler JW. Mindfulness training improves working memory capacity and GRE performance while reducing mind wandering. Psychol Sci. 2013; 24 (5):776-81. Epub 2013/03/30. https://doi.org/10.1177/0956797612459659 PMID: 23538911.

210. Neece CL. Mindfulness-Based Stress Reduction for Parents of Young Children with Developmental Delays: Implications for Parental Mental Health and Child Behavior Problems. J Appl Res Intellect Disabil. 2014; 27(2):174-86. https://doi.org/10.1111/jar.12064 PMID: 23813562. Language: English. Entry Date: 20140212. Revision Date: 20150710. Publication Type: Journal Article.

211. Dennis ML. The Influence of Parental Mental Health on Child Outcomes: The Role of the Parenting Process. Ann Arbor: Loma Linda University; 2016.

212. Chan N, Neece CL. Parenting Stress and Emotion Dysregulation among Children with Developmental Delays: The Role of Parenting Behaviors. J Child Fam Stud. 2018; 27:4071-82. https://doi.org/10. 1007/s10826-018-1219-9

213. Norouzi E, Gerber M, Masrour FF, Vaezmosavi M, Pühse U, Brand S. Implementation of a mindfulness-based stress reduction (MBSR) program to reduce stress, anxiety, and depression and to improve psychological well-being among retired Iranian football players. Psychol Sport Exerc. 2020; 47:101636. https://doi.org/10.1016/j.psychsport.2019.101636

214. Nyklicek I, Kuijpers KF. Effects of mindfulness-based stress reduction intervention on psychological well-being and quality of life: Is increased mindfulness indeed the mechanism? Ann Behav Med. 2008; 35(3):331-40. http://dx.doi.org/10.1007/s12160-008-9030-2. PMID: 18535870.

215. O'Donnell RMR. Pilot RCT of Mindfulness-Based Stress Reduction (MBSR) Versus Progressive Muscle Relaxation (PMR) to Reduce Symptoms of Distress Among Elderly Dementia Caregivers: Results at One Year Post-Intervention. Ann Arbor: The University of Arizona; 2017.

216. O'Donnell RMM. Mindfulness-based stress reduction as an intervention among family caregivers of persons with neurocognitive disorders. Ann Arbor: The University of Arizona; 2013.

217. Oken BS, Fonareva I, Haas $\mathrm{M}$, Wahbeh $\mathrm{H}$, Lane JB, Zajdel D, et al. Pilot controlled trial of mindfulness meditation and education for dementia caregivers. Journal of Alternative \& Complementary Medicine —New York. 2010; 16 (10):1031-8. https://doi.org/10.1089/acm.2009.0733 PMID: 20929380

218. Pan WL, Gau ML, Lee TY, Jou HJ, Liu CY, Wen TK. Mindfulness-based programme on the psychological health of pregnant women. Women Birth. 2018; 32(1):e102-e9. http://dx.doi.org/10.1016/j. wombi.2018.04.018. PMID: 29752225.

219. Pan W, Chang C, Chen S, Gau M, et al. Assessing the effectiveness of mindfulness-based programs on mental health during pregnancy and early motherhood - a randomized control trial. BMC Pregnancy Childbirth. 2019; 19 (1):346. https://doi.org/10.1186/s12884-019-2503-4 PMID: 31601170

220. Park JM, Choi IR. The Effect of K-MBSR Program on Stress, Stress Coping Style, Depression, Anger and Sleep of Middle Aged Women. [Korean]. J Korean Acad Nurs. 2016; 46(2):194-206. http://dx.doi. org/10.4040/jkan.2016.46.2.194. PMID: 27182016. 
221. Perez-Blasco J, Viguer $P$, Rodrigo M. Effects of a mindfulness-based intervention on psychological distress, well-being, and maternal self-efficacy in breast-feeding mothers: results of a pilot study. Arch Womens Ment Health. 2013; 16(3):227-36. https://doi.org/10.1007/s00737-013-0337-z PMID: 23512648. Language: English. Entry Date: 20130530. Revision Date: 20150711. Publication Type: Journal Article.

222. Perez-Blasco J, Sales A, Meléndez JC, Mayordomo T. The Effects of Mindfulness and Self-Compassion on Improving the Capacity to Adapt to Stress Situations in Elderly People Living in the Community. Clin Gerontol. 2016; 39(2):90-103. https://doi.org/10.1080/07317115.2015.1120253 PMID: 113083307. Language: English. Entry Date: 20170818. Revision Date: 20170823. Publication Type: Article.

223. Phang CK, Mukhtar F, Ibrahim N, Keng S-L, Mohd Sidik S. Effects of a brief mindfulness-based intervention program for stress management among medical students: the Mindful-Gym randomized controlled study. Adv Health Sci Educ. 2015; 20(5):1115-34. https://doi.org/10.1007/s10459-015-9591-3 PMID: 25697124. Language: English. Entry Date: 20170202. Revision Date: 20180620. Publication Type: journal article.

224. Pipe TB, Bortz JJ, Dueck A, Pendergast D, Buchda V, Summers J. Nurse leader mindfulness meditation program for stress management: a randomized controlled trial. J Nurs Adm. 2009; 39(3):130-7. https://doi.org/10.1097/NNA.0b013e31819894a0 PMID: 19590469. Language: English. Entry Date: 20090522. Revision Date: 20150818. Publication Type: Journal Article.

225. Plummer C, Cloyd E, Doersam JK, Dietrich MS, Hande KA. Mindfulness in a Graduate Nursing Curriculum: A Randomized Controlled Study. Holist Nurs Pract. 2018; 32(4):189-95. https://doi.org/10. 1097/HNP.0000000000000277 PMID: 29894374. Language: English. Entry Date: 20180705. Revision Date: 20180705. Publication Type: Article.

226. Hande KA, Cloyd E, Doersam J, Dietrich MS, Plummer C. Mindfulness Centered Stress Reduction in a Graduate Nursing Curriculum: A Randomized Controlled Study. Tenn Nurse. 2017; 80(3):13-22. PMID: 125099763. Language: English. Entry Date: 20170913. Revision Date: 20180612. Publication Type: Article.

227. Pots WTM, Meulenbeek PAM, Veehof MM, Klungers J, Bohlmeijer ET. The efficacy of mindfulnessbased cognitive therapy as a public mental health intervention for adults with mild to moderate depressive symptomatology: A randomized controlled trial. PLoS ONE. 2014; 9(10):e109789. http://dx.doi. org/10.1371/journal.pone.0109789. PMID: 25333885.

228. Prakash RS, Whitmoyer $P$, Bredemeier K, Fountain-Zaragoza S. Mindfulness Training and Cognitive Control in Older Adults: Results of a Single-Blind, Placebo-Controlled Randomized Controlled Trial. Gerontologist. 2015; 55.

229. Whitmoyer P, Fountain-Zaragoza S, Andridge RBK, Londerée A, Kaye L, et al. Mindfulness Training and Attentional Control in Older Adults: a Randomized Controlled Trial. Mind. 2020; 11:203-18. https://doi.org/10.1007/s12671-019-01218-3.

230. Richards D Jr, M WE. The Effects of a Brief Mindfulness Intervention on Self-Compassion Among Undergraduate College Students. VISTAS. 2012; 1 (3):1-10.

231. Richards D. A randomized control study of brief mindfulness with undergraduates on self-compassion, mindfulness, and thought suppression. Ann Arbor: Northern Arizona University; 2013.

232. Richards D Jr, M WE. Thought Suppression and Mindfulness: A Randomized Controlled Study. VISTAS Online. 2013; 65:1-10.

233. Robins CJ, Keng SL, Ekblad AG, Brantley JG. Effects of mindfulness-based stress reduction on emotional experience and expression: A randomized controlled trial. J Clin Psychol. 2012; 68(1):117-31. http://dx.doi.org/10.1002/jclp.20857. PMID: 22144347.

234. Keng SL, Smoski MJ, Robins CJ, Ekblad AG, Brantley JG. Mechanisms of change in mindfulnessbased stress reduction: Self-compassion and mindfulness as mediators of intervention outcomes. $J$ Cogn Psychother. 2012; 26(3):270-80. http://dx.doi.org/10.1891/0889-8391.26.3.270. PMID: 366319758.

235. Roeser RW, Schonert-Reichl KA, Jha A, Cullen M, Wallace L, Wilensky R, et al. Mindfulness training and reductions in teacher stress and burnout: Results from two randomized, waitlist-control field trials. J Educ Psychol. 2013; 105 (3):787-804. https://doi.org/10.1037/a0032093

236. Taylor C, Harrison J, Haimovitz K, Oberle E, Thomson K, Schonert-Reichl K, et al. Examining ways that a mindfulness-based intervention reduces stress in public school teachers: A mixed-methods study. Mind. 2016; 7(1):115-29. 2015-32654-001.

237. Crain TL, Schonert-Reichl KA, Roeser RW. Cultivating teacher mindfulness: Effects of a randomized controlled trial on work, home, and sleep outcomes. J Occup Health Psychol. 2017; 22(2):138-52. https://doi.org/10.1037/ocp0000043 PMID: 27182765. Language: English. Entry Date: In Process. Revision Date: 20171105. Publication Type: journal article. 
238. Sampl J, Maran T, Furtner MR. A Randomized Controlled Pilot Intervention Study of a MindfulnessBased Self-Leadership Training (MBSLT) on Stress and Performance. Mind. 2017; 8(5):1393-407. Epub 2017/10/11. https://doi.org/10.1007/s12671-017-0715-0 PMID: 28989553.

239. Schellekens M, Van den Hurk D, Prins J, Donders A, Molema J, Dekhuijzen R, et al. MindfulnessBased Stress Reduction Added to Care as Usual for Lung Cancer Patients and Their Partners: A Randomized Controlled Trial. J Thorac Oncol. 2017; 12(1):S1416-S7.

240. Schellekens MPJ, Hurk DGM, Prins JB, Donders ART, Molema J, Dekhuijzen R, et al. Mindfulnessbased stress reduction added to care as usual for lung cancer patients and/or their partners: A multicentre randomized controlled trial. Psychooncology. 2017; 26(12):2118-26. https://doi.org/10.1002/ pon.4430 PMID: 28337821. Language: English. Entry Date: 20180720. Revision Date: 20180420. Publication Type: journal article.

241. Schellekens MPJ, van den Hurk DGM, Prins JB, Donders ART, Molema J, Dekhuijzen R, et al. Mindfulness-Based Stress Reduction in Addition to Treatment as Usual for Patients with Lung Cancer and Their Partners: Results of a Multi-Centre Randomized Controlled Trial. Int J Behav Med. 2016; 23: S231-S.

242. Schroeder DA, Stephens E, Colgan D, Hunsinger M, Rubin D, Christopher MS. A Brief MindfulnessBased Intervention for Primary Care Physicians: A Pilot Randomized Controlled Trial. Am J Lifestyle Med. 2018; 12(1):83-91. https://doi.org/10.1177/1559827616629121 PMID: 30202383

243. Sevinc G, Holzel BK, Hashmi J, Greenberg J, McCallister A, Treadway M, et al. Common and Dissociable Neural Activity After Mindfulness-Based Stress Reduction and Relaxation Response Programs. Psychosom Med. 2018; 80(5):439-51. Epub 2018/04/12. https://doi.org/10.1097/PSY. 0000000000000590 PMID: 29642115.

244. Shapiro SL, Schwartz GE, Bonner G. Effects of Mindfulness-Based Stress Reduction on Medical and Premedical Students. J Behav Med. 1998; 21 (6):581-99. https://doi.org/10.1023/a:1018700829825 PMID: 9891256

245. Shapiro SL, Astin JA, Bishop SR, Cordova M. Mindfulness-Based Stress Reduction for Health Care Professionals: Results From a Randomized Trial. Int J Stress Manag. 2005; 12 (2):164-76. https://doi. org/10.1037/1072-5245.12.2.164

246. Shapiro P, Lebeau R, Tobia A. Mindfulness Meditation for Medical Students: a Student-Led Initiative to Expose Medical Students to Mindfulness Practices. Medical Science Educator. 2019; 29 (2):43951. https://doi.org/10.1007/s40670-019-00708-2

247. Shearer A, Hunt M, Chowdhury M, Nicol L. Effects of a brief mindfulness meditation intervention on student stress and heart rate variability. Int J Stress Manag. 2016; 23(2):232-54. https://doi.org/10. 1037/a0039814

248. Smart CM, Segalowitz SJ. Respond, don't react: The influence of mindfulness training on performance monitoring in older adults. Cogn Affect Behav Neurosci. 2017; 17(6):1151-63. https://doi.org/10.3758/ s13415-017-0539-3 PMID: 28971360

249. Smart CM, Segalowitz SJ, Mulligan BP, Koudys J, Gawryluk JR. Mindfulness Training for Older Adults with Subjective Cognitive Decline: Results from a Pilot Randomized Controlled Trial. J Alzheimers Dis. 2016; 52 (2):757-74. https://doi.org/10.3233/JAD-150992 PMID: 27060951

250. $\square$ tefan CA, Căpraru C, Szilágyi M. Investigating effects and mechanisms of a mindfulness-based stress reduction intervention in a sample of college students at risk for social anxiety. Mind. 2018; 9 (5):1509-21. https://doi.org/10.1007/s12671-018-0899-y 2018-06755-001.

251. Steinberg BA, Klatt M, Duchemin A-M. Feasibility of a Mindfulness-Based Intervention for Surgica Intensive Care Unit Personnel. Am J Crit Care. 2016; 26(1):10-8. https://doi.org/10.4037/ ajcc2017444 PMID: 27965223.

252. Duchemin AM, Steinberg BA, Marks DR, Vanover K, Klatt M. A small randomized pilot study of a workplace mindfulness-based intervention for surgical intensive care unit personnel: Effects on salivary aamylase levels. J Occup Environ Med. 2015; 57 (4):393-9. https://doi.org/10.1097/JOM. 0000000000000371 PMID: 25629803

253. Klatt M, Steinberg B, Duchemin AM. Mindfulness in motion (Mim): An onsite mindfulness based intervention (MBI) for chronically high stress work environments to increase resiliency and work engagement. J Vis Exp. 2015; 101:1-11. https://doi.org/10.3791/52359 PMID: 26168365

254. Klatt M, Norre C, White S. P04.38. The efficacy of an English-to-Danish translation of a low-dose mindfulness workplace intervention for Scandinavian bank employees on stress. BMC Complement Altern Med. 2012; 12(Suppl 1):1-. https://doi.org/10.1186/1472-6882-12-s1-p308 PMID: 104481674. Language: English. Entry Date: 20121129. Revision Date: 20150711. Publication Type: Journal Article.

255. Strub L, Tarquinio C. [Mindfulness-Based Cognitive Therapy (MBCT) program with workers in an industrial setting: a pilot study]. Sante Ment Que. 2013; 38(1):207-25. https://doi.org/10.7202/ 1019193ar PMID: 24336997. 
256. Thomas J, Raynor M, Bahussain E. Stress reactivity, depressive symptoms, and mindfulness: A Gulf Arab perspective. International Perspectives in Psychology: Research, Practice, Consultation. 2016 5(3):156-66. https://doi.org/10.1037/ipp0000055 2016-21448-001.

257. van Berkel J, Boot CRL, Proper KI, Bongers PM, van der Beek AJ. Effectiveness of a worksite mindfulness-based multi-component intervention on lifestyle behaviors. Int J Behav Nutr Phys Act. 2014; 11:1-21. https://doi.org/10.1186/1479-5868-11-1 PMID: 24405936. Language: English. Entry Date: 20140224. Revision Date: 20150710. Publication Type: Journal Article.

258. van Berkel J, Proper KI, Boot CR, Bongers PM, van der Beek AJ. Mindful 'Vitality in Practice': an intervention to improve the work engagement and energy balance among workers; the development and design of the randomised controlled trial. BMC Public Health. 2011; 11(1):736-. PMID: 104593182. Language: English. Entry Date: 20130222. Revision Date: 20150711. Publication Type: Journal Article.

259. van Berkel J, Boot CRL, Proper KI, Bongers PM, van der Beek AJ. Process Evaluation of a Workplace Health Promotion Intervention Aimed at Improving Work Engagement and Energy Balance. J Occup Environ Med. 2013; 55(1):31-8. https://doi.org/10.1097/JOM.0b013e318269e5a6 PMID: 23147554

260. Berkel Jv, Boot CRL, Proper KI, Bongers PM, Beek AJvd. Effectiveness of a Worksite MindfulnessRelated Multi-Component Health Promotion Intervention on Work Engagement and Mental Health: Results of a Randomized Controlled Trial. PLoS ONE. 2014; 9(1):e84118. https://doi.org/10.1371/ journal.pone.0084118 PMID: 24489648

261. Van Berkel J, Boot CRL, Proper KI, Bongers PM, Van Der Beek AJ. Correction: Effectiveness of a worksite mindfulness-related multi-component health promotion intervention on work engagement and mental health: Results of a randomized controlled trial. PLoS ONE.2015; 10(3):e0122428. http:// dx.doi.org/10.1371/journal.pone.0122428. PMID: 25811187.

262. van Dongen JM, van Berkel J, Boot CR, Bosmans JE, Proper KI, Bongers PM, et al. Long-Term Cost-Effectiveness and Return-on-Investment of a Mindfulness-Based Worksite Intervention: Results of a Randomized Controlled Trial. Journal of occupational and environmental medicine / American College of Occupational and Environmental Medicine. 2016; 58(6):550-60. Epub 2016/06/ 10. https://doi.org/10.1097/JOM.0000000000000736 PMID: 27281638.

263. Van Dam NT, Hobkirk AL, Sheppard SC, Aviles-Andrews R, Earleywine M. How does mindfulness reduce anxiety, depression, and stress? An exploratory examination of change processes in wait-list controlled mindfulness meditation training. Mind. 2014; 5(5):574-88. https://doi.org/10.1007/s12671013-0229-3 2013-20100-001.

264. van Dijk I, Lucassen P, Akkermans RP, van Engelen BGM, van Weel C, Speckens AEM. Effects of Mindfulness-Based Stress Reduction on the Mental Health of Clinical Clerkship Students: A ClusterRandomized Controlled Trial. Acad Med. 2017; 92(7):1012-21. https://doi.org/10.1097/ACM. 0000000000001546 PMID: 28121650

265. van Dijk I, Lucassen $P$, Akkermans $R$, van Engelen B, van Weel C, Speckens A. Effect of Mindfulness Training on the Course of Psychological Distress and Positive Mental Health of Medical Students during Their Clinical Clerkships. A Cluster-Randomized Controlled Trial. Int J Behav Med. 2016; 23:S86-S.

266. Verweij $\mathrm{H}$, van Ravesteijn $\mathrm{H}$, van Hooff MLM, Lagro-Janssen ALM, Speckens AEM. MindfulnessBased Stress Reduction for Residents: A Randomized Controlled Trial. J Gen Intern Med. 2018; 33 (4):429-36. https://doi.org/10.1007/s11606-017-4249-x PMID: 29256091

267. Verweij $\mathrm{H}$, van Ravesteijn HJ, van Hooff MLM, Lagro ALM, Speckens AEM. Mindfulness-Based Stress Reduction for Medical Residents: Effects on Burnout and Wellbeing. A Randomized Controlled Trial. Int J Behav Med. 2016; 23:S86-S.

268. Vieten C, Astin J. Effects of a mindfulness-based intervention during pregnancy on prenatal stress and mood: results of a pilot study. Arch Womens Ment Health. 2008; 11(1):67-74. Epub 2008/03/05. https://doi.org/10.1007/s00737-008-0214-3 PMID: 18317710.

269. Vinesett AL, Price M, Wilson $\mathrm{KH}$. Therapeutic Potential of a Drum and Dance Ceremony Based on the African Ngoma Tradition. J Altern Complement Med. 2015; 21(8):460-5. https://doi.org/10.1089/acm. 2014.0247 PMID: 26171652. Language: English. Entry Date: 20150730. Revision Date: 20160801. Publication Type: Journal Article.

270. Wang Y, Xin T-T, Liu X-H, Zhang Y, Lu H-H, Zhai Y-B. Mindfulness can reduce automatic responding: Evidences from Stroop task and prospective memory task. Acta Psychol Sin. 2012; 44(9):1180-8. https://doi.org/10.3724/sp.j.1041.2012.01180 2012-29206-005.

271. Whitebird RR, Kreitzer MJ, Crain AL, Lewis BA, Hanson LR, Enstad CJ. Mindfulness-based stress reduction for family caregivers: A randomized controlled trial. Gerontologist. 2013; 53(4):676-86. https://doi.org/10.1093/geront/gns126 PMID: 23070934 2013-25784-012.

272. Whitebird RR, Kreitzer MJ, Lewis BA, Hanson LR, Crain AL, Enstad CJ, et al. Recruiting and retaining family caregivers to a randomized controlled trial on mindfulness-based stress reduction. Contemp Clin Trials. 2011; 32(5):654-61. http://dx.doi.org/10.1016/j.cct.2011.05.002. PMID: 21601010. 
273. Williams KA, Kolar MM, Reger BE, Pearson JC. Evaluation of a Wellness-Based Mindfulness Stress Reduction Intervention: A Controlled Trial. Am J Health Promot. 2001; 15 (6):422-32. https://doi.org/ 10.4278/0890-1171-15.6.422 PMID: 11523499

274. Wilson DM. Effects of mindfulness-based art processing (MBAP) on the well-being and job performance of working adults: evaluating a novel intervention: Institute of Transpersonal Psychology; 2012.

275. Wong C, Yip BHK, Gao T, Lam KYY, Woo DMS, Yip ALK, et al. Mindfulness-Based Stress Reduction (MBSR) or Psychoeducation for the Reduction of Menopausal Symptoms: A Randomized, Controlled Clinical Trial. Sci Rep. 2018; 8 (1). https://doi.org/10.1038/s41598-018-24945-4 PMID: 29700350

276. Woolhouse H, Mercuri K, Judd F, Brown SJ. Antenatal mindfulness intervention to reduce depression, anxiety and stress: A pilot randomised controlled trial of the MindBabyBody program in an Australian tertiary maternity hospital. BMC Pregnancy Childbirth. 2014; 14(1):369. http://dx.doi.org/10.1186/ s12884-014-0369-z. PMID: 612230531.

277. Xu W, Wang Y-Z, Liu X-H. Effectiveness of 8-week mindfulness training improving negative emotions. Chin Ment Health J. 2015; 29(7):497-502. 2015-35092-004.

278. Yazdanimehr R, Omidi A, Sadat Z, Akbari H. The Effect of Mindfulness-integrated Cognitive Behavior Therapy on Depression and Anxiety among Pregnant Women: a Randomized Clinical Trial. J Caring Sci. 2016; 5(3):195-204. https://doi.org/10.15171/jcs.2016.021 PMID: 27752485.

279. Yazdanimehr R, Omidi A, Akbari H, Sadat Z. Mindfulness Training and Quality of Life Among Pregnant Women: A Randomized Clinical Trial. Nurs Midwifery Stud. 2017; 6(2).

280. Zhang JY, Cui YX, Zhou YQ, Li YL. Effects of mindfulness-based stress reduction on prenatal stress, anxiety and depression. Psychol Health Med. 2018; 24 (1):51-8. https://doi.org/10.1080/13548506. 2018.1468028 PMID: 29695175

281. Cohen J. Statistical power analysis for the behavioral sciences. 2nd ed. Hillsdale, NJ: Lawrence Erlbaum Associates; 1988.

282. Jackson D, Riley R, White IR. Multivariate meta-analysis: potential and promise. Stat Med. 2011; 30 (20):2481-98. Epub 2011/01/27. https://doi.org/10.1002/sim.4172 PMID: 21268052.

283. Goldberg SB, Tucker RP. Allegiance effects in mindfulness-based interventions for psychiatric disorders: A meta-re-analysis. Psychotherapy research: journal of the Society for Psychotherapy Research. 2019:1-10. Epub 2019/09/12. https://doi.org/10.1080/10503307.2019.1664783 PMID: 31506031.

284. Kreplin U, Farias M, Brazil IA. The limited prosocial effects of meditation: A systematic review and meta-analysis. Sci Rep. 2018; 8(1):2403. Epub 2018/02/07. https://doi.org/10.1038/s41598-01820299-z PMID: 29402955.

285. Büttner F, Winters M, Delahunt E, Elbers R, Lura CB, Khan KM, et al. Identifying the 'incredible'! Part 2: Spot the difference-a rigorous risk of bias assessment can alter the main findings of a systematic review. Br J Sports Med. 2019; 54 (13):801-8. https://doi.org/10.1136/bjsports-2019-101675 PMID: 31871014

286. de Bruin M. Risk of bias in randomised controlled trials of health behaviour change interventions: Evidence, practices and challenges. Psychol Health. 2015; 30 (1):1-7. https://doi.org/10.1080/08870446. 2014.960653 PMID: 25223823

287. Rosenkranz MA, Dunne JD, Davidson RJ. The next generation of mindfulness-based intervention research: what have we learned and where are we headed? Curr Opin Psychol. 2019; 28:179-83. Epub 2019/02/11. https://doi.org/10.1016/j.copsyc.2018.12.022 PMID: 30739006.

288. Carmody J, Baer RA. How long does a mindfulness-based stress reduction program need to be? A review of class contact hours and effect sizes for psychological distress. J Clin Psychol. 2009; 65 (6):627-38. Epub 2009/03/25. https://doi.org/10.1002/jclp.20555 PMID: 19309694.

289. Baer R, Crane C, Miller E, Kuyken W. Doing no harm in mindfulness-based programs: Conceptual issues and empirical findings. Clin Psychol Rev. 2019. Epub 2019/01/15. https://doi.org/10.1016/j.cpr. 2019.01.001 PMID: 30638824

290. Huijbers MJ, Crane RS, Kuyken W, Heijke L, van den Hout I, Donders ART, et al. Teacher Competence in Mindfulness-Based Cognitive Therapy for Depression and Its Relation to Treatment Outcome. Mindfulness (N Y). 2017; 8(4):960-72. Epub 2017/08/02. https://doi.org/10.1007/s12671-0160672-z PMID: 28757901.

291. Galante J, Stochl J, Dufour G, Vainre M, Wagner AP, Jones PB. Effectiveness of providing university students with a mindfulness-based intervention to increase resilience to stress: one-year follow-up of a pragmatic randomised controlled trial. J Epidemiol Community Health. In press. http://dx.doi.org/10. 1136/jech-2020-214390.

292. Goldberg SB, Tucker RP, Greene PA, Davidson RJ, Wampold BE, Kearney DJ, et al. Mindfulnessbased interventions for psychiatric disorders: A systematic review and meta-analysis. Clin Psychol Rev. 2018; 59:52-60. Epub 2017/11/12. https://doi.org/10.1016/j.cpr.2017.10.011 PMID: 29126747. 
293. Ruiz-Î́niguez R, Santed Germán MÁ, Burgos-Julián FA, Díaz-Silveira C, Carralero MA. Effectiveness of mindfulness-based interventions on anxiety for children and adolescents: A systematic review and meta-analysis. Early Interv Psychiatry. 2020; 14 (3):263-74. https://doi.org/10.1111/eip.12849 PMID: 31287618

294. Wong SYS, Chan JYC, Zhang D, Lee EKP, Tsoi KKF. The Safety of Mindfulness-Based Interventions: a Systematic Review of Randomized Controlled Trials. Mind. 2018; 9 (5):1344-57. https://doi. org/10.1007/s12671-018-0897-0

295. Burrows L. Safeguarding Mindfulness Meditation for Vulnerable College Students. Mindfulness [Internet]. 2016; 7:[284-5 pp.]. Available from: https://doi.org/10.1007/s12671-015-0434-3

296. Dobkin PL, Irving JA, Amar S. For Whom May Participation in a Mindfulness-Based Stress Reduction Program be Contraindicated? Mind. 2011; 3 (1):44-50. https://doi.org/10.1007/s12671-011-0079-9

297. Schlosser M, Sparby T, Voros S, Jones R, Marchant NL. Unpleasant meditation-related experiences in regular meditators: Prevalence, predictors, and conceptual considerations. PLoS ONE. 2019; 14 (5):e0216643. Epub 2019/05/10. https://doi.org/10.1371/journal.pone.0216643 PMID: 31071152.

298. Cebolla A, Demarzo M, Martins $P$, Soler J, Garcia-Campayo J. Unwanted effects: Is there a negative side of meditation? A multicentre survey. PLoS ONE. 2017; 12(9):e0183137. Epub 2017/09/06. https://doi.org/10.1371/journal.pone.0183137 PMID: 28873417.

299. Ioannidis JPA. The Mass Production of Redundant, Misleading, and Conflicted Systematic Reviews and Meta-analyses. Milbank Q. 2016; 94 (3):485-514. https://doi.org/10.1111/1468-0009.12210 PMID: 27620683

300. Petticrew M, Anderson L, Elder R, Grimshaw J, Hopkins D, Hahn R, et al. Complex interventions and their implications for systematic reviews: a pragmatic approach. J Clin Epidemiol. 2013; 66(11):120914. Epub 2013/08/21. https://doi.org/10.1016/j.jclinepi.2013.06.004 PMID: 23953085.

301. Stanley S, Kortelainen I. Assembling mindful bodies: mindfulness as a universal 'laboratory of practice'. In: Salmenniemi S, Nurmi J, Perheentupa I, Bergroth H, editors. Assembling Therapeutics: Cultures, Politics and Materiality: Routledge; 2019. p. 20-42.

302. Perez S. Meditation and mindfulness apps continue their surge amid pandemic: Tech Crunch; 2020 [09-11-2020]. Available from: https://techcrunch.com/2020/05/28/meditation-and-mindfulness-appscontinue-their-surge-amid-pandemic/.

303. Campion J. Public mental health commissioning guidance: Embedding mental health in local public health work. Perspect Public Health. 2013; 133:87-9. https://doi.org/10.1177/1757913913476631 PMID: 23467527 